\title{
Frequency-domain P-approximant filters for time-truncated inspiral gravitational wave signals from compact binaries
}

\author{
Thibault Damour ${ }^{1}$, Bala R. Iyer ${ }^{2,3,4}$ and B.S. Sathyaprakash ${ }^{3}$ \\ 1 Institut des Hautes Etudes Scientifiques, 91440 Bures-sur-Yvette, France \\ ${ }^{2}$ Raman Research Institute, Bangalore 560 080, India \\ ${ }^{3}$ Cardiff University, P.O. Box 913, Cardiff, CF2 3YB, U.K. \\ ${ }^{4}$ Albert Einstein Institute, D-14476, Golm, Germany
}

(May 28, 2018)

\begin{abstract}
Frequency-domain filters for time-windowed gravitational waves from inspiralling compact binaries are constructed which combine the excellent performance of our previously developed timedomain P-approximants with the analytic convenience of the stationary phase approximation without a serious loss in event rate. These Fourier-domain representations incorporate the "edge oscillations" due to the (assumed) abrupt shut-off of the time-domain signal caused by the relativistic plunge at the last stable orbit. These new analytic approximations, the SPP-approximants, are not only effectual for detection and faithful for parameter estimation, but are also computationally inexpensive to generate (and are faster by factors up to 10, as compared to the corresponding time-domain templates). The SPP approximants should provide data analysts the Fourier-domain templates for massive black hole binaries of total mass $m \lesssim 40 M_{\odot}$, the most likely sources for LIGO and VIRGO.
\end{abstract}

\section{INTRODUCTION AND SUMMARY}

The discovery of the first binary pulsar in 1974 [1] has had a very important impact on gravitational wave research. First, it proved the reality of gravitational radiation by measuring the orbital period decay [2] entailed by the propagation at the velocity of light of the gravitational interaction between the two neutron stars making up the system [3]. Second, it provided the first experimental evidence that General Relativity correctly describes gravity in the strong-field regime [4]. Third, it led to a shift in perception regarding the most promising sources for future gravitational wave $(\mathrm{GW})$ detectors, away from the then assumed, violent - but less predictable - gravitational collapse associated with supernovae, to the more predictable, final inspiralling phase of compact binaries of neutron stars and black holes driven by gravitational radiation-reaction. This also led to the thrust in the laser interferometric gravitational wave detectors which are inherently broad-band rather than in the narrow-band bar detectors.

\section{A. Data analysis algorithms for inspiral wave searches}

Consider a compact binary system like the binary pulsar after it has been inspiralling inwards for three hundred million years due to gravitational radiation-reaction. The inspiral waveform enters the detector bandwidth during the last few minutes of evolution of the binary. Our ability, in principle, to compute the waveform very accurately, allows us to track the gravitational wave phase and enhance the signal-to-noise ratio by integrating the signal for the interval during which it lasts in the detector band. This, in turn, requires a template with which the detector output may be filtered. Though template waveforms should, optimally, be exact copies of the expected signal, in practice, they are constructed by some approximation scheme and will differ from the actual signal in the detector output. Consequently, the overlap of template and signal waveforms will be less than if they had exactly matched, leading to a loss of potential events. Data analysis issues like these for inspiralling compact binaries of neutron stars and black holes have been formulated and addressed for the last twelve years [5.,6], even though interferometric gravitational wave detectors like the GEO600 [7] or LIGO [8] and VIRGO [9] are a year or three in the future. Much of the work in this area has addressed practical issues of direct relevance to data analysis strategies. These include: construction of templates for detection [10], the number of templates, their placement, spacing, the required computing power and the storage or memory requirement 11], the order of post-Newtonian (PN) approximation adequate for detection [12 14], parameter estimation by covariance matrix 15 17] and Monte Carlo simulations [18], determination of cosmological parameters 19], tests of general relativity 20], one step versus hierarchical searches [21], effects of precession [22] and of eccentricity [17,23]. For the time-domain waveform, all of these works use the restricted post-Newtonian 
approximation to quasi-circular inspiral. This keeps the crucial phase information to the best order of approximation then available 24, but restricts the amplitude to be Newtonian and the harmonic to the second harmonic of the orbital frequency. Such an approximation should be adequate for the on-line search of gravitational wave signals [25]. Evidently, it is assumed that the offline analysis of the data will use the best available (unrestricted post-Newtonian) representation of the inspiral signals.

\section{B. Modelling inspiral waveforms}

The post-Newtonian approximation is basically a Taylor expansion (in powers of $v / c$ ) and all the above treatments use as building blocks the straightforward Taylor expansions in $v / c$ of some intermediate quantities (orbital energy and gravitational-wave flux). We shall refer to the templates based on such straightforward PN expansions as "Taylor approximants" (or simply T-approximants). The very slow convergence and oscillatory behaviour of the PN expansion, and therefore of the sequence of Taylor approximants, made imperative a search for better approximants for phasing. This prompted us [13 (later referred to as DIS) to propose new approximants, with much improved convergence properties, for application to gravitational-wave data analysis problems.

In DIS [13], we showed how to construct a new type of time-domain approximant, called "P-approximants", which not only converged faster and more monotonically, but were also more effectual (larger overlaps for detection) and faithful (smaller biases for parameter estimation) than the standard T-approximants. Our construction was twopronged: on the one hand, it introduced new basic energy and flux functions, and on the other hand, it made a systematic use of Padé techniques (a well-known convergence-acceleration technique) to construct successive approximants of our new basic energy and flux functions. These new functions form a pivotal aspect of our construction and successfully handle issues related to appearance of non-rational functions in the energy function and logarithmic terms in the flux function that for long proved to be hurdles to the application of well-known Padé techniques to this problem. For initial LIGO, the 2.5PN P- approximants are likely to provide overlaps in excess of $96.5 \%$ with exact waveforms so that more than $90 \%$ of the potential events can be detected. In contrast, the corresponding $2.5 \mathrm{PN}$ Taylor approximants can only detect about $50 \%$ of the potential events for massive systems (at the price of large biases $\sim 15 \%$ ). Later studies have confirmed the performance of these P-approximants [27] and assessed [28] their need in related contexts of space based interferometers like LISA.

\section{Fourier representation of inspiral signals and validity of the stationary phase approximation (SPA)}

Independent of the choice between T- and P-approximants, another desirable approximation in data analysis for inspiralling compact binaries is the stationary phase approximation (SPA), which is a simple, explicit analytic approximation to the Fourier transform of the time-domain chirp (see, e.g., [29]). In fact, most work on inspiral waveforms (except DIS) has used only SPA approximants to the frequency-domain chirps. In the course of our P-approximant work we noticed a progressive worsening of the overlap between the SPA and the "exact" Fourier transform - numerically computed by a fast Fourier transform (FFT) of the time-domain signal — (see Table II of DIS) and commented on these 'inaccuracies of the SPA'. In the above by SPA one means not only the problem of the formal accuracy of the stationary phase estimate to the Fourier transform of an analytically extended, mathematical signal but also some issues linked to the physics, and observability, of the real signal. In particular, in DIS, we were considering templates which are shut off, in the time-domain, at the last stable orbit (LSO). The present paper will also consider such time-truncated inspiral signals. We shall discuss this point in more detail below, but the idea is that the post-inspiral signal (plunge + merger) will have a frequency content very different from the inspiral one (probably pushed to much higher frequencies). It should, therefore make sense to try to construct filters that represent as best as possible an inspiral signal which lasts only up to some maximum time (time-windowing). For such signals, DIS noted a worsening of the usual (frequency-windowed) SPA approximation, both as the total mass of the system increases and as the post-Newtonian approximation order is increased, and mentioned that this worsened performance was due to the fact that "such systems emit many less wave cycles in the effective detector bandwidth" centered (for initial LIGO) near

\footnotetext{
${ }^{1}$ This statement was proven in DIS by quantifying the convergence of the sequence of P-approximants towards some 'fiducial exact' waveform. In the test-particle case this waveform used the known Schwarzschild's energy function $E(v)$ and Poisson's numerically computed GW flux 26]. In the comparable mass case, it was constructed by modelling the $\eta$ - dependent higher post-Newtonian corrections to the best known analytical results.
} 
$f_{\text {det }}=167 \mathrm{~Hz}$. In this paper $f_{\text {det }}$ denotes the frequency at which the noise power spectrum per logarithmic bin of the detector is the least (or equivalently the frequency at which the detector is most sensitive to a broad-band burst). To avoid irrelevant, uncontrolled sources of inaccuracy, DIS used the FFT of the time-windowed chirp rather than its SPA to generate the frequency-domain waveform and make comparisons between the T- and P-approximants.

The use of FFT rather than SPA in DIS makes the P-approximant computationally expensive. As will be discussed in detail in Sec. VI, the use of SPA or similar frequency-domain representations is far less expensive. The obvious need to incorporate this desirable feature makes urgent and mandatory a critical investigation of the possibility of marrying together the excellent performance of the P-approximants to the relative inexpensiveness of the SPA without a serious loss in event rate.

Recently, some issues related to the accuracy of the SPA have been investigated. For general chirps, ChassandeMottin and Flandrin [30] have studied whether the usual conditions assumed for the validity of the SPA are necessary and sufficient and attempted a quantitative control of the approximation. Droz et al [31] have examined other issues related to the accuracy of the SPA of particular relevance to gravitational wave data analysis. Unlike DIS, by SPA, Droz et al imply only the stationary phase estimate of the Fourier transform and discuss separately the issue of windowing - the fact that the signal in the time-domain lasts only from $t_{\min }$ to $t_{\max }$ or a time-window. To improve the SPA estimate of a Newtonian chirp, they compute the next order contribution? (to the Fourier integral) by the method of steepest descent, show that it is of order $v_{\mathcal{M}}^{10}$ relative to the leading order SPA estimate and conclude that it is small enough to be justifiably neglected. [Here $v_{\mathcal{M}}$ is an invariantly defined 'velocity' related to the instantaneous gravitational wave frequency $F$ and chirp mass $\mathcal{M}$ by $v_{\mathcal{M}}=(\pi \mathcal{M} F)^{1 / 3}$. The chirp mass is related to the total mass $m=m_{1}+m_{2}$ and dimensionless mass ratio $\eta=\frac{m_{1} m_{2}}{\left(m_{1}+m_{2}\right)^{2}}$ by $\mathcal{M}=\eta^{3 / 5} m$.] They point out the importance of windowing, estimate the amplitude and phase modulations induced in the frequency-domain by the time-window and conclude that in all cases these modulations have negligible effect on overlaps. However, their analytic expression for the effects of windowing is only valid for values of frequencies well away from the boundaries of the natural frequency-window induced by the time-window, denoted by $F_{\min }=F\left(t_{\min }\right)$ and $F_{\max }=F\left(t_{\max }\right)-$ the gravitational wave frequencies at times $t_{\min }$ and $t_{\max }$, respectively. In this paper we provide a formalism allowing one to compute analytic approximations to the Fourier-transform of a time-windowed signal in the most crucial edgefrequency-domains $f \sim F_{\min }$ and $f \sim F_{\max }$ (including $f<F_{\min }$ and $f>F_{\max }$ ). As first noticed in DIS and discussed in detail in this present work, the effect of window oscillations on overlaps (claimed to be negligible in [31]) starts to be noticeable when the total mass $m \gtrsim 13 M_{\odot}$ and becomes very significant for $m \gtrsim 20 M_{\odot}$. [Here we consider equal mass systems $\eta=1 / 4$.] Since the difference between the statements in DIS and Droz et al [31] can be disconcerting and a serious source of confusion to the potential user community, we discuss this in further detail next.

In DIS, what was meant in Table II (the only place where it was used) by "stationary phase approximation" was the product of the usual SPA by a simple Heaviside step function $\theta\left(F_{\max }-f\right)$ i.e. $\tilde{h}(f)$ was truncated above a Fourier frequency $f=F_{\max }$ where $F_{\max }$ is the instantaneous gravitational wave frequency at which the time-domain signal is itself terminated, assumed to be (in DIS and here) the frequency at the last stable orbit $F_{\mathrm{LSO}}$. [In the following, we shall, for brevity, refer to this frequency Windowed Usual Stationary Phase Approximation as the 'uSPAw'.] We were motivated to do this from the stationary phase result itself. The SPA (to the Fourier transform of the chirp) says that the dominant contribution to a certain Fourier amplitude $\tilde{h}(f)$ comes from a neighbourhood of time (in the Fourier integral) when the instantaneous frequency $F(t)$ numerically reaches the corresponding Fourier frequency $f$. It is therefore to be expected that the signal essentially terminates at $f=F_{\mathrm{LSO}}$ i.e. that there is no significant power in the Fourier transform of the signal beyond $F_{\mathrm{LSO}}$. This is indeed true in the first approximation, as is evident from Fig. 6 below, which shows that the power in the exact Fourier transform of the time-windowed signal [computed via a discrete Fourier transform (DFT)] falls off much faster than the SPA for $f>F_{\mathrm{LSO}}$. Moreover, as is discussed in detail below, in the relativistic case the usual SPA breaks down at $F_{\mathrm{LSO}}$ and cannot be meaningfully extended for $f>F_{\text {LSO }}$. Hence the values quoted in Table II of DIS were obtained by computing the overlap of the DFT of the truncated time-domain waves with the truncated SPA representation of the wave.

On the other hand, a critical examination of Droz et al [31] reveals that their claim regarding the adequacy of the SPA in fact has only a restricted domain of validity. It is relevant to SPA considered as a mathematical algorithm to be applied to a generic smooth signal and low mass binaries $\left(m \lesssim 13 M_{\odot}\right)$. As acknowledged by the authors, they do not address physical issues related to an eventual time-domain cut-off of the signal at $F_{\text {LSO }}$. What they call "Newtonian signals" are unphysical, formally defined chirps whose instantaneous frequencies are extended to

\footnotetext{
${ }^{2}$ We shall give below the general result for any chirp.

${ }^{3}$ Note that, following DIS, we shall use $v \equiv(\pi m F)^{1 / 3}$, instead of $v_{\mathcal{M}}=\eta^{1 / 5} v$, in all our analysis. We also use units such that $G=c=1$.
} 
$F_{\max }=F_{\text {Nyquist }} \gg F_{\mathrm{LSO}}$, in fact, better described as 'analytically extended Newtonian signals'. It is the SPA of this formal, analytically extended signal which is shown to produce overlaps with exact FFTs better than 0.99 even for massive binary systems of chirp mass $\mathcal{M}=10 M_{\odot}$, corresponding to a total mass of $m \sim 23 M_{\odot}$ for an equal mass system $(\eta=1 / 4)$. These large overlaps, in our view, are not a proof of the validity of the SPA to compute, physically relevant, accurate frequency-domain inspiral templates, as they do not address the important issue of inspiral-signal termination at or near the $F_{\mathrm{LSO}}$ when $F_{\mathrm{LSO}} \sim f_{\text {det }}$, the frequency at which the broad-band noise of the detector is the least. It turns out that for binary systems of total mass $m \gtrsim 28 M_{\odot}$ the power in the Fourier domain beyond $f=F_{\text {LSO }}$ for a relativistic signal, is a significant fraction $(>10 \%)$ of the total power. If the usual (frequency-windowed) SPA is used in constructing frequency-domain inspiral waves we are risking the loss of more than $30 \%$ of the events from binaries with masses $\gtrsim 28 M_{\odot}$. [This will be illustrated in Fig. 目 below.] This is in addition to the losses induced by the inaccuracy of the post-Newtonian waveforms and the discreteness of the bank of templates used in data analysis.

\section{Massive black hole binaries and first detections in LIGO/VIRGO}

Let us first establish our notation. We define the Fourier transform (FT) $\tilde{h}(f)$ of a time-domain signal $h(t)$ by

$$
h(t)=\int_{-\infty}^{\infty} d f e^{-2 \pi i f t} \tilde{h}(f) ; \tilde{h}(f)=\int_{-\infty}^{\infty} d t e^{2 \pi i f t} h(t) .
$$

We write the (suitably transformed) output of the detector as

$$
h_{\text {out }}=h(t)+n(t),
$$

where $h(t)$ is the signal and $n(t)$ the noise. The correlation function of the noise reads

$$
\overline{n\left(t_{1}\right) n\left(t_{2}\right)}=C_{n}\left(t_{1}-t_{2}\right)=\int_{-\infty}^{\infty} d f S_{n}(f) e^{2 \pi i f\left(t_{1}-t_{2}\right)},
$$

where $S_{n}(f)=S_{n}(-f)$ is the two-sided noise power spectral density. In all the present work, we shall consider a noise curve of the type expected for initial interferometers. For initial LIGO we take [32],

$$
\begin{aligned}
S_{n}(f) & =\frac{S_{0}}{2}\left[2+2\left(\frac{f}{f_{0}}\right)^{2}+\left(\frac{f}{f_{0}}\right)^{-4}\right], f \geq f_{s}, \\
& =\infty, f<f_{s} .
\end{aligned}
$$

with $f_{s}=40 \mathrm{~Hz}, f_{0}=200 \mathrm{~Hz}$ and $S_{0}=1.47 \times 10^{-46} \mathrm{~Hz}^{-1}$. In the above we have included a factor of $1 / 2$ $\left[S_{n}^{\text {one-sided }} \equiv 2 S_{n}^{\text {two-sided }}\right]$ because Eq. (1.4a) gives the two-sided noise; the one-sided noise would be given by the same formula without the factor of $1 / 2$. The minimum of $S_{n}(f)$ is at $f=f_{0}$ and is equal to $S_{\min }=2.5 S_{0}$. However, a physically more relevant quantity is the minimum of the dimensionless quantity $h_{n}^{2}(f) \equiv f S_{n}(f)$ (effective GW noise, see below). This is reached at the characteristic detection frequency $f=f_{\mathrm{det}}=0.8347 f_{0}$, and is equal to $\left(h_{n}^{\min }\right)^{2}=2.2761 f_{0} S_{0}$. The above numerical value for $f_{0}$ and $S_{0}$ leads to $f_{\text {det }}=167 \mathrm{~Hz}$ and corresponds to $h_{n}^{\min }=2.5868 \times 10^{-22}$.

For VIRGO on the other hand, the corresponding noise curve is given by

$$
\begin{aligned}
S_{n}(f) & =\frac{S_{0}}{2}\left[10^{3}\left(\frac{f_{s}}{f}\right)^{5}+2\left(\frac{f_{0}}{f}\right)+1+\left(\frac{f}{f_{0}}\right)^{2}\right], f \geq f_{s}, \\
& =\infty, f<f_{s},
\end{aligned}
$$

In this case, $f_{s}=20 \mathrm{~Hz}, f_{0}=500 \mathrm{~Hz}$ while $S_{0}=3.24 \times 10^{-46} \mathrm{~Hz}^{-1}$ [33]. The minimum of $h_{n}(f)=\sqrt{f S_{n}(f)}$ is reached at $f=103 \mathrm{~Hz}$ and is equal to $h_{n}^{\min }=4.2902 \times 10^{-22}$. It should be noted that the VIRGO noise curve is used only in this Section, while discussing Fig. 1. In the rest of the paper and all the Figures and Tables, the scalar product is defined using the LIGO noise curve.

Anticipating on formulas to be discussed in IIB, the square of the signal to noise ratio (SNR) is given by

$$
\rho^{2}=\left(\frac{S}{N}\right)^{2}=\frac{\langle k, h\rangle^{2}}{\langle k, k\rangle},
$$


where the scalar product is defined by

$$
\langle k, h\rangle \equiv \int_{-\infty}^{+\infty} d f \frac{\tilde{k}^{*}(f) \tilde{h}(f)}{S_{n}(f)} .
$$

Here, $h$ denotes the exact signal, and $k$ the filter used in the data analysis. We assume in this paper that the signal $h$ is given by a time-truncated adiabatic inspiral signal. [For simplicity, we consider in this subsection Newtonian waveforms, and we approximate the Fourier transform of a time-truncated Newtonian signal by the very accurate improved Newtonian stationary phase approximation (inSPA) to be constructed below.] In computing $\rho^{2}$ we average over all the angles (determining both the detector and the source orientations), and we place the source at a fiducial distance of $100 \mathrm{Mpc}$. [Note that a coalescence rate of $10^{-5}$ per galaxy and per year implies that in two years one event should happen within $100 \mathrm{Mpc}$.

In most of the literature one uses as Fourier-domain filter $\tilde{k}(f)$ the frequency-windowed usual stationary phase approximation (uSPAw) to estimate the SNR for an inspiral signal. We illustrate in Fig. 1 the loss in signal strength extracted by using as filter the uSPAw in LIGO and VIRGO [cf. Eqs. (1.4a) and (1.5a)], instead of using the optimal filter $k=h$ (leading to the optimal SNR $\rho^{2}=\langle h, h\rangle$ ). The plot also shows on the top horizontal axis the last stable orbit frequency corresponding to the total mass in question. The left vertical axis shows the SNR extracted and the right vertical axis shows the sensitivity, $h_{n}^{-1} \equiv\left[f S_{n}(f)\right]^{-1}$, (both of which are dimensionless) of LIGO-I and VIRGO instruments. While reading the sensitivity curve one should use the top and right axes and while reading the SNR curve one should use the bottom and left axes. The SNR values plotted in Fig. 1 have been computed numerically by inserting the relevant values of $\tilde{h}(f)$ and $\tilde{k}(f)$ in Eq. (1.6). Though we did not use it, it might help the reader to see the analytical expression of $\rho^{2}$ obtained in the simple approximation where $\tilde{k}(f) \simeq \tilde{h}(f) \simeq \tilde{h}^{\text {uspaw }}(f)$. Using the equations of Sec. II below (and averaging over angles as explained in Sec. IV A below) leads to

$$
\left\langle\rho^{2}\right\rangle \simeq \frac{\eta}{15 \pi}\left(\frac{m}{d}\right)^{2} \int_{0}^{F_{\mathrm{LSO}}} \frac{d f}{f} \frac{1}{v(f)} \frac{1}{f S_{n}(f)}
$$

where $\langle.$.$\rangle denotes the angular average, d$ the distance to the source, $v(f)=(\pi m f)^{1 / 3}$ and $S_{n}(f)$ the two-sided noise given by Eq. (1.4a). We indicated no precise detection threshold in Fig. 1 because this depends on many parameters (like the number of detectors involved). The reader should, however, have in mind that a reasonable detection threshold is, at least, $\rho_{\text {threshold }} \sim 5$.

We note that the effective sensitivity of LIGO-I peaks near a frequency of $167 \mathrm{~Hz}$ which is the last stable orbit frequency for a binary of total mass of about $27 M_{\odot}$. The effective sensitivity of VIRGO peaks at a much lower frequency of $103 \mathrm{~Hz}$. This low-frequency sensitivity of VIRGO means two important things: Firstly, lighter binaries (i.e., $m \lesssim 30 M_{\odot}$ ) are integrated for a longer time in the low frequency regime and, therefore, the corrections to the Fourier transform introduced in this paper are less important for such systems. This means that the uSPAw is quite good in extracting the full signal power of such binaries as evidenced in Fig. 11. Notice that, for VIRGO, the uSPAw curve follows the inSPA curve for $m \lesssim 30 M_{\odot}$. On the contrary, LIGO's lower sensitivity to lower frequencies makes it important to include the corrections to the Fourier transform of LSO-truncated signals from binaries of mass $m \gtrsim 15 M_{\odot}$. In LIGO's case uSPAw extracts only $75 \%$ of the full SNR implying a loss of more than $40 \%$ of all massive binary coalescences. On the other hand, the low-frequency peak of VIRGO sensitivity means that we will have to employ the accurate Fourier domain models discussed in this paper for more massive binaries, i.e. $m \gtrsim 30 M_{\odot}$. [Note, however, that the low-frequency sensitivity of VIRGO means that, for low-mass and medium-mass binaries, it is even more crucial to use P-approximants (instead of the usually considered T-approximants) than for LIGO, in order to accurately keep track of the phasing of the many cycles accumulated at low frequencies.]

It is fair to say that at present the most well-understood gravitational waveform is the inspiral one and thus the only reliable templates correspond to inspiral signals. It is also generally believed that binary black holes are better candidates for gravitational wave sources than binary neutron stars due to their larger masses (the average mass of observed black hole candidates is around $8 M_{\odot}$ [34]). Theoretical computations based on stellar evolution indicate that binary black holes with individual masses $\lesssim 15 M_{\odot}$ may be the only known sources that exist (hopefully) in sufficient numbers 35 38]. When looking at Fig. 1, one clearly sees the importance of dealing with binary black holes with total masses in the range of $28-30 M_{\odot}$. They lead to signals with the best SNR. However, it is precisely for such systems that the $F_{\mathrm{LSO}}$ is around the middle of the detection bandwidth for initial LIGO i.e. $F_{\mathrm{LSO}} \sim f_{\text {det }}$. The most likely sources to detect are in the problematic region discussed in this paper. This makes it imperative to not lose SNR when dealing with such signals and provides the other major motivation for our work. If our assumption that the best models of inspiral waveforms must be abruptly shut off in the time domain holds, it is essential to use the improved SPA formulas discussed in this work in order to maximise our chances of detecting inpiralling binaries. The analysis presented in this paper provides insights and techniques to deal with binary black hole signals in probably the most crucial mass range. 


\section{E. Summary of the present paper and proposals for data analysis groups}

In this paper we propose analytical approximations to the Fourier-transform of the LSO-truncated time-domain inspiral waves that are very accurate even for the massive black hole binaries, the most likely sources for LIGO and VIRGO [overlaps with FFT $>0.99$ for $m \lesssim 40 M_{\odot}$ ] and are at the same time computationally inexpensive. We call our final new, frequency-domain filters the SPP approximants because they combine the computational convenience of stationary phase approximants with the accuracy of the (time-domain) P-approximants. Our strategy is two-fold: On the one hand we introduce a correction factor $\mathcal{C}(f)$ to the usual SPA for $f<f_{\text {up }} \leq F_{\text {LSO }}$ which improves the SPA by taking into account the "edge" oscillations present when $f \lesssim f_{\text {up }}$. ( $f_{\text {up }}$ will be defined below. For Newtonian-like signals $f_{\text {up }}=F_{\mathrm{LSO}}$, while for relativistic signals $f_{\text {up }}<F_{\mathrm{LSO}}$.) On the other hand, we introduce a new approximation to the Fourier transform for $f>f_{\text {up }}$ which efficiently recovers the signal power around and beyond the frequency corresponding to the last stable orbit. These features are important new steps forward as there was no formalism until now that could compute (especially for relativistic signals) Fourier transforms analytically for $f \sim F_{\mathrm{LSO}}$, and in particular $f>F_{\mathrm{LSO}}$. These new features now make it possible to generate templates directly in the Fourier-domain, leading to a saving on the computational cost of template generation by a factor of 10 or more.

Our concrete proposal to the interferometer data analysis groups that are building the gravitational wave search software and wish to have Fourier-domain filters which are both accurate and fast-computed, is thus the following: First, we confirm that for accurate post-Newtonian template generation of binary systems of total mass $m \lesssim 40 M_{\odot}$ one needs to use a frequency-domain version of the P-approximant (previously defined only in the time-domain). For $m<5 M_{\odot}$ a straightforward (uncorrected for edge-effects) SPA of the P-approximants is sufficient. (They match with the exact DFT of the same time-signal with overlaps $>0.999$.) On the other hand, in the total mass range $5 M_{\odot} \lesssim m \lesssim 40 M_{\odot}$, and assuming that one wishes an accurate frequency-domain (f-domain) representation of a time-windowed signal it is crucial to use our new SPP approximants. For $m \gtrsim 40 M_{\odot}$ a straightforward DFT is recommended (but, anyway, the signal is not known with enough precision in this high mass range, where the plunge and merger signals become observationally important).

It is important to stress the position we assume in this paper: Given the absence of any detailed and precise information about the plunge signal today, we suggest that a time-truncated chirp (time-windowed signal) is currently our best bet and the modified SPA presented in this paper is the appropriate Fourier-domain representation one must use. However, this should not be taken to imply that we are claiming to have logically excluded the other possibility that the f-window may turn out to be the better choice, when we have further details about the transition from inspiral to plunge and, about the plunge waveform. Even so, we emphasize that a definitive contribution of the present work is to provide explicitly for the first time the frequency-domain version of the time-domain P-approximants which were shown in DIS to bring indispensible improvements over the usually considered T-approximants. Consequently, even in the unlikely case where a straightforward frequency-window turns out to be a better model than the time-window assumed in most of this work, one will still require the formulas given in this paper [with the trivial change of replacing the correction factors $\mathcal{C}(\zeta)$ by a $\theta$ function $\theta\left(F_{\mathrm{LSO}}-f\right)$ ] to generate sufficiently accurate $\mathrm{f}$-domain filters. Thus this work may not be the complete final answer but only a step ahead and a partial contribution towards defining good f-domain filters. Assumptions that seem the best we can accept, require special tools for their analysis and this paper provides them.

This paper is organised as follows: In Sec. II A, IIB, II C as a prelude to later technical material, we introduce several useful physical notions and employ them to give a preliminary discussion of the questions raised by the detectability of massive-binary signals. In Sec. II D we summarise the mathematical tools used in the paper to estimate the timetruncated chirps. In Sec. III] we consider time-windowed Newtonian-like signals. Sec. III A provides a short summary of the well known SPA. Sec. III B sets up the basic equations to discuss the Fourier transform of time-windowed signals. Sec. III estimates the edge contribution to the Fourier transform coming from the non-resonant integral. This is followed by Sec. IIID and IIIE where we elaborate in detail the construction of optimal analytic approximations to the Fourier transform of the time-windowed gravitational wave chirp (improved Newtonian SPA). In Sec. IIIF we compare and contrast in detail the usual SPA with our improved SPA for Newtonian-like signals. Sec. IV A addresses the new issues related to the Fourier-transform of time-windowed relativistic signals. In Sec IV B we present a new method to estimate the small non-resonant contribution in the relativistic case. In Sec. IV $\bar{q}$ we construct a new form of improved SPA for such signals (improved relativistic SPA). Combining this improved relativistic SPA with the P-approximants of DIS leads to the construction of the frequency-domain SPP approximants. In Sec. V we use the SPP approximants constructed earlier and investigate their faithfulness and effectualness in detail for the test mass case. Based on this we comment on the corresponding situation in the comparable mass case. In Sec. VI we compare the computing costs for template generation using the time-domain FFT with corresponding costs for the frequency-domain SPA and improved SPA both for Newtonian and relativistic cases. Sec. VII contains our concluding remarks. 


\section{PRELIMINARY DISCUSSION}

As a preface to the technical treatments of the following Sections in which we shall construct optimal analytic approximations to the Fourier transform of the gravitational wave inspiral signal $h(t)$ let us start by discussing some general issues which are central to this paper.

\section{A. Wiener filters and time-truncated inspiral signals}

We briefly recall the principle underlying the optimal linear filter technique (Wiener filter). A (real) linear filter is a linear functional of the detector's output, $h_{\text {out }}$, Eq.(1.2), say

$$
K\left[h_{\mathrm{out}}\right]=\int_{-\infty}^{\infty} d t K(t) h_{\mathrm{out}}(t)=\int_{-\infty}^{\infty} d f \tilde{K}(-f) \tilde{h}_{\mathrm{out}}(f)=\int_{-\infty}^{\infty} d f \tilde{K}^{*}(f) \tilde{h}_{\mathrm{out}}(f)
$$

Let us associate to any $K(t)$ the time-domain function $k(t)$ such that its FT equals $\tilde{k}(f) \equiv S_{n}(f) \tilde{K}(f)$ and let us introduce the Wiener scalar product (defined on real time-domain functions)

$$
\begin{gathered}
\langle g, h\rangle \equiv \int_{-\infty}^{\infty} \frac{d f}{S_{n}(f)} \tilde{g}^{*}(f) \tilde{h}(f)=\int_{-\infty}^{\infty} \int_{-\infty}^{\infty} d t_{1} d t_{2} g\left(t_{1}\right) w_{1}\left(t_{1}-t_{2}\right) h\left(t_{2}\right), \\
\text { where, } w_{1}(\tau)=\int_{-\infty}^{\infty} \frac{d f}{S_{n}(f)} e^{2 \pi i f \tau}=w_{1}(-\tau)
\end{gathered}
$$

is the convolution inverse of the noise correlation function $C_{n}(\tau)=C_{n}(-\tau)$ i.e.

$$
\left(w_{1} * C_{n}\right)(t)=\delta(t)
$$

[Here $*$ denotes the convolution product: $\left.(g * h)(t) \equiv \int_{-\infty}^{\infty} d \tau g(\tau) h(t-\tau)\right]$. With this notation the action of the filter $K$ on $h_{\text {out }}$ reads

$$
K\left[h_{\text {out }}\right]=\left\langle k, h_{\text {out }}\right\rangle \equiv S+N,
$$

where $S$ is the filtered 'signal' and $N$ the filtered 'noise', defined by

$$
S \equiv K[h]=\langle k, h\rangle ; N \equiv K[n]=\langle k, n\rangle
$$

The definition of the symmetric Wiener scalar product Eq. 2.2) is such that the statistical average of a product of filtered noises simplify: $\overline{\left\langle k_{1}, n\right\rangle\left\langle k_{2}, n\right\rangle}=\overline{\left\langle k_{1}, n\right\rangle\left\langle n, k_{2}\right\rangle}=\left\langle k_{1}, k_{2}\right\rangle$. In particular, the variance of the filtered noise $N$ reads $\overline{N^{2}}=\overline{\langle k, n\rangle^{2}}=\langle k, k\rangle$, so that the square of the signal-to-noise ratio (SNR) for the filter defined by any function $k(t)$ reads

$$
\rho^{2} \equiv \frac{S^{2}}{\overline{N^{2}}}=\frac{\langle k, h\rangle^{2}}{\langle k, k\rangle}=|\mathcal{O}(k, h)|^{2}\langle h, h\rangle
$$

where we have defined the 'overlap', or normalized ambiguity function, between $k$ and $h$

$$
\mathcal{O}(k, h) \equiv \frac{\langle k, h\rangle}{\sqrt{\langle k, k\rangle\langle h, h\rangle}}
$$

Schwarz's inequality guarantees that $|\mathcal{O}(k, h)| \leq 1$, the equality being reached only when $k(t)=\lambda h(t)$. (We work here in the space of real signals.) For a given signal $h(t)$, the choice of filter $K \leftrightarrow k$ which maximizes the SNR $\rho$ is, in view of Eq. (2.7), $k(t)=\lambda h(t)$, where the proportionality constant is unimportant and can be taken to be one ('Wiener Theorem'). This optimal linear filter theorem applies when the full time-development of the signal $h(t)$ is known, the noise is stationary and has a known spectral distribution $S_{n}(f)$.

It is important to note that, saying that the best 'associated' filter $k(t)$ is simply $k(t)=h(t)$, means that the best time-domain filter $K(t)$, which must be directly correlated with the detector's output, $K\left[h_{\text {out }}\right]=\int_{-\infty}^{\infty} d t K(t) h_{\text {out }}(t)$, is a non-local (in time) functional of $h(t)$. Explicitly, 


$$
K(t)=\left(w_{1} * h\right)(t)=\int_{-\infty}^{\infty} d \tau w_{1}(\tau) h(t-\tau)
$$

This poses the question whether, for an off-line analysis of the data, one would like to store a bank of these non-local time-domain filters $K(t)$, which densely cover the expected parameter space. In the present paper, we shall assume that one anyway computes (nearly) on-line the Fast Fourier Transform (FFT) of the detector's output (which is anyway needed to factor out the frequency dependent effect of the interferometer on the GW signal), and we shall set ourselves the task of providing the best possible analytical representations of the Fourier transform of the expected signals $\tilde{h}(f)$. Moreover, the availability of a fast Fourier algorithm makes the filtering problem computationally less intensive in the Fourier-domain. It is well-known that the computation of a discrete correlation for all (discrete) time lags between the output and the filter [ie the discrete version of Eq. 2.1) requires $\mathcal{O}\left[N^{2}\right.$ ] operations in the time-domain while it takes only $\mathcal{O}\left[N \log _{2}(N)\right]$ operations in the Fourier domain (because a time lag $\tau$ adds a factor $\exp (2 \pi i f \tau)$ in the f-domain version of Eq. (2.1), which is equivalent to computing a certain inverse Fourier transform). Discrete correlation in the f-domain suffers from spurious correlations for non-zero time lags but this is easily taken care of by padding the tail part of a template with large number of zeroes (see, eg. Ref [6] for details.)

As the problem of the locality/non-locality in time will be crucial to our discussion of the inspiral signals, we wish to give an alternative discussion of Wiener's optimal linear filter theorem. Indeed, another proof of the theorem can be obtained by introducing a 'whitening' transformation say $w_{\frac{1}{2}}$, which simplifies the properties of the noise. We define the 'whitening-kernel' $w_{\frac{1}{2}}(\tau)$ by

$$
w_{\frac{1}{2}}(\tau)=\int_{-\infty}^{+\infty} \frac{d f}{\sqrt{S_{n}(f)}} e^{2 \pi i \tau f} ; \tilde{w}_{\frac{1}{2}}(f) \equiv \frac{1}{\sqrt{S_{n}(f)}} .
$$

For any function $g(t)$, the action of the kernel $w_{\frac{1}{2}}$ on $g$ (i.e. the 'whitened' version of the function $g$ ) can be denoted by

$$
g_{\frac{1}{2}}(t) \equiv\left(w_{\frac{1}{2}} * g\right)(t)=\int_{-\infty}^{\infty} d \tau w_{\frac{1}{2}}(\tau) g(t-\tau)
$$

The name 'whitening kernel' comes from the fact that the transformed noise $n_{\frac{1}{2}}(t)$ is 'white' i.e. uncorrelated:

$$
\overline{n_{\frac{1}{2}}\left(t_{1}\right) n_{\frac{1}{2}}\left(t_{2}\right)}=\int_{-\infty}^{\infty} d f e^{2 \pi i f\left(t_{1}-t_{2}\right)}=\delta\left(t_{1}-t_{2}\right) .
$$

Note that $w_{\frac{1}{2}}$ is simply the convolution square-root of the Wiener kernel $w_{1}$ introduced above: $w_{\frac{1}{2}} * w_{\frac{1}{2}}=w_{1}$. The Wiener theorem states then that, after having whitened all the functions, the optimal filter is simply the usual straightforward correlation between the (whitened) output and the (whitened) signal i.e.

$$
K_{\text {optimal }}\left[h_{\text {out }}\right]=\int_{-\infty}^{\infty} d t h_{\frac{1}{2}}(t) h_{\frac{1}{2}}^{\text {out }}(t)
$$

where $h_{\frac{1}{2}}=w_{\frac{1}{2}} * h, h_{\frac{1}{2}}^{\text {out }}=w_{\frac{1}{2}} * h^{\text {out }}$. In other words, we can think of the optimal filter as being local-in-time after the application, to all signals, of the convolution kernel $w_{\frac{1}{2}}$. When working with the transformed time-domain functions $h_{\frac{1}{2}}(t), h_{\frac{1}{2}}^{\text {out }}(t) \cdots$, we shall say that we work in the 'whitened time-domain'. In this language, the best filter in the whitened time-domain is to simply correlate (as when trying to visually superpose two time-functions) the output with a copy of the signal. This 'whitened time domain' is conceptually useful in the present context because it introduces only a small non-locality (by small we mean here much smaller than the non-locality introduced by the Fourier transformation). Indeed, as the function $1 / \sqrt{S_{n}(f)}$ has a rather flat maximum, its Fourier transform $w_{\frac{1}{2}}(\tau)$ is nearly a delta function as seen in Fig. 目 wherein we have plotted the whitening kernel for the initial LIGO interferometer. More precisely, $w_{\frac{1}{2}}(\tau)$ is an even function made of a positive spike around $\tau=0$, followed (on each side) by a slightly negative wing which decays fast towards zero as $|\tau| \rightarrow+\infty$. The half-width at half-maximum of the central spike is $0.18 \mathrm{~ms}$. The location of the wings is around $\tau= \pm 0.002 \mathrm{~s}$. Therefore the non-locality contained in the whitening transformation is only between $0.2 \mathrm{~ms}$ and $2 \mathrm{~ms}$ (depending on the function on which it acts). This non-locality together with the one and a half cycle in $w_{\frac{1}{2}}$ is sufficient to efficiently damp both high and low frequencies so that $h_{\frac{1}{2}}=w_{\frac{1}{2}} * h$ is a chirp whose amplitude is important only when the instantaneous frequency is around $f_{\mathrm{p}}=165 \mathrm{~Hz}$ (see below). We shall use below this whitened time domain picture to discuss the important features of the expected chirp that we should try to model as well as possible. 
In the present paper, we shall be primarily interested in massive compact binaries with total mass $m=m_{1}+m_{2}$ in the approximate range $3 M_{\odot} \lesssim m \lesssim 40 M_{\odot}$. We recall that the GW signal from a compact binary is made of an inspiral signal, followed, after the last stable orbit is reached, by a plunge signal which leads to a final merger signal. Thanks to the analytical work on the motion [3] of and GW emission [24,39] from general relativistic binary systems we have quite a good analytical control of the inspiral signal. In the present paper, we shall further argue that we have also a rather good analytical control on the location of the last stable orbit (LSO), i.e. on the transition between the inspiral and plunge. First, DIS [13] introduced a new, more robust approach to the determination of LSO based on the invariant function $e(v)$. More recently [40], a new approach to the dynamics of binary systems has confirmed the result of DIS (saying that the LSO was slightly more 'inwards' for comparable mass systems than as predicted by the test-mass limit) and predicts values for the important physical quantities at the LSO (notably the orbital frequency) which are even nearer to the (Schwarzschild-like) ones obtained in the test-mass limit. We anticipate that further analytical progress in the problem of motion of binary systems [41], combined with the LSO-determination techniques given in [13] and [40,42] will soon allow one to know with more certainty and more precision the gravitational wave frequency at the LSO. Pending such a determination, we shall use as fiducial value for the GW frequency at the LSO — when we need it for simple analytical estimates - the usual 'Schwarzschild-like' approximation

$$
F_{\mathrm{LSO}}=4400\left(\frac{M_{\odot}}{m}\right) \mathrm{Hz} .
$$

However, in our actual numerical calculations and plots we shall use the $\eta$-dependent $F_{\text {LSO }}$ corresponding to the approximation used for the energy function $E_{\mathrm{A}}(v)$. For instance, in the case of the $2 \mathrm{PN} \mathrm{P}$-approximant $P_{4}$ we have 13 .

$$
F_{\mathrm{LSO}}^{P_{4}}=4397.2\left(\frac{1+\frac{1}{3} \eta}{1-\frac{35}{36} \eta}\right)^{\frac{3}{2}}\left[2-\frac{1+\frac{1}{3} \eta}{\sqrt{1-\frac{9}{16} \eta+\frac{1}{36} \eta^{2}}}\right]^{\frac{3}{2}} \frac{M_{\odot}}{m} \mathrm{~Hz} .
$$

In the equal-mass case $(\eta=1 / 4)$ this yields $F_{\mathrm{LSO}}=5719.4 M_{\odot} / \mathrm{m} \mathrm{Hz}$. Note that the most recent determination of the LSO [40] suggests that when $\eta \neq 0$ the GW frequency at the LSO lies between Eq. (2.14) and Eq. (2.15):

$$
F_{\mathrm{LSO}}=4397.2(1+0.3155 \eta)\left(\frac{M_{\odot}}{m}\right) \mathrm{Hz} .
$$

Preliminary studies indicate that the plunge signal, emitted during the fast fall of the two masses towards each other following the crossing of the LSO, will last (when $4 \eta \sim 1$ ) only for a fraction of an orbital period (see 40 and [43)|'As. As usual, one can also assume that the subsequent merger signal linked to the formation of a black hole of total mass $\sim m$ contains significantly higher frequencies than the inspiral ones. Indeed, the characteristic frequency of the merger signal may be taken to be given by the real part of the most slowly damped quadrupolar normal mode of a black hole (which when neglecting the black hole spin, has a complex circular frequency $\omega_{1} m_{\mathrm{bh}}=0.37367-0.08896 i$ 44]), i.e. (with $m_{\mathrm{bh}} \sim m$ )

$$
f_{\text {merger }} \sim f_{\text {bh }} \simeq \frac{0.374}{2 \pi m} \simeq 12000 \frac{M_{\odot}}{m} \mathrm{~Hz} .
$$

Eqs. (2.14) and (2.17) lead us to accept that there is a significant frequency separation between the inspiral and plunge signals and the merger one: $f_{\text {merger }} / F_{\mathrm{LSO}} \sim 2.75$. Therefore, if we restrict our attention to systems such that the characteristic detection frequency $f_{\text {det }}$ defined by the noise curve, stays logarithmically nearer to $F_{\mathrm{LSO}}$ than to $f_{\text {merger }}$, it seems plausible that a good filter to use for GW detection can neglect the (ill-known) merger signal, but should try to model as accurately as possible the inspiral and plunge signal. For the initial LIGO noise curve, Eq. (1.4a), the characteristic detection frequency $f_{\text {det }}$ is $167 \mathrm{~Hz}$. It is then for a total mass $m \leq 43.5 M_{\odot}$ that $f_{\text {det }} / F_{\mathrm{LSO}} \leq f_{\mathrm{bh}} / f_{\text {det }}$.

We shall see below that, just before reaching the LSO, the inspiral signal is still significantly 'quasi-periodic' (with $\gtrsim 6$ cycles before a significant change in instantaneous frequency). By contrast, though the plunge signal may not decay monotonically and may be oscillating, it seems reasonable to assume that the plunge lasts only for a fraction of

\footnotetext{
${ }^{4}$ We assume here that we are in the generic case where the spins of the coalescing objects are smallish compared to their maximal Kerr value.
} 
the orbital period $T_{\mathrm{LSO}}=2 F_{\mathrm{LSO}}^{-1}$. Thus, in the absence of a precise knowledge of the plunge signal, a good model of the time-domain signal consists in abruptly shutting off, by a step function $\theta\left(t_{\mathrm{LSO}}-t\right)$ the (adiabatic) inspiral signal beyond the time $t_{\mathrm{LSO}}$ when the last stable orbit is reached. We also tested formally the robustness of our approach by showing that our model above has a good overlap with a signal which decays smoothly on a time scale of a few (up to 3) $F_{\mathrm{LSO}}^{-1}$ beyond the LSO. Because of the likely oscillatory behaviour of the plunge signal, details of the oscillations are necessary for any further improvements and we are currently working towards improving our understanding of the transition between the inspiral and the plunge 43.

These considerations motivate us to propose that, in the absence of knowledge of the optimal filter which should be $k_{\text {optimal }}(t)=k_{\text {exact }}(t)$, our best bet is to use as (sub-optimal) filter the time-truncated inspiral signal $h_{\text {inspiral }}(t) \theta\left(t_{\mathrm{LSO}}-\right.$ $t$ ). In other words, we think that the best strategy is to use all the information available, in the time-domain, about the signal and to replace the transient plunge and higher frequency merger signals by zero, as a measure of our current ignorance. But having settled on this tactic in the time-domain, the aim of this paper is to provide the best possible frequency-domain description of such a time-windowed signal. We shall see in detail below that the Fourier transform $\tilde{h}_{\mathrm{tw}}(f)=\mathrm{FT}\left[h_{\mathrm{tw}}(t)\right]$ of the time-windowed signal $h_{\mathrm{tw}}(t) \equiv h_{\text {inspiral }}(t) \theta\left(t_{\mathrm{LSO}}-t\right)$ has a non-trivial structure which is not captured by the usually considered frequency-windowed stationary phase approximation $\tilde{h}_{\text {spaw }}(f)$. In particular, for massive systems, a significant fraction of the total power is contained in the 'tail' of $\tilde{h}_{\text {tw }}(f)$ beyond $f=F_{\mathrm{LSO}}$. The general result Eq. (2.7) for SNR obtained with any filter then says that the Fourier-domain filter $\tilde{h}_{\mathrm{tw}}(f)$, though sub-optimal, should be still significantly better than $\tilde{h}_{\text {spaw }}(f)$ because (under our assumptions about the plunge + merger signal) it has better overlaps with the exact signal. We shall return below to this important issue and give further arguments (in the time-domain) to confirm the superiority of $\tilde{h}_{\mathrm{tw}}(f)$ over $\tilde{h}_{\mathrm{spaw}}(f)$ (see Fig. 9 and Fig. 10 and text around it).

\section{B. The number of useful cycles}

Often one mentions that, in the total time development of an inspiral signal, the total number of gravitational wave cycles

$$
N_{\mathrm{tot}}=\frac{1}{2 \pi}\left(\phi_{\mathrm{end}}-\phi_{\mathrm{begin}}\right)=\int_{F_{\mathrm{beg}}}^{F_{\mathrm{end}}} d F\left(\frac{1}{2 \pi} \frac{d \phi}{d F}\right)
$$

is very large. Here $\phi$ is the gravitational wave phase, $\phi_{\text {end }}$ is the phase at the end of the inspiral regime (defined by the last stable orbit for sufficiently massive systems, i.e. for the black-hole-neutron-star and the black-hole-blackhole systems), while $\phi_{\text {begin }}$ is the phase when the signal enters the lower frequency (seismic) cutoff of the detector bandwidth. We have also rewritten $N_{\text {tot }}$ as an integral over the running instantaneous gravitational wave frequency $F$. However, the large number $N_{\text {tot }}$, Eq. (2.18), is not significant because the only really useful cycles are those which contribute most to the signal-to-noise ratio (SNR). To have a clearer idea of what one might want to mean by the notion of a useful number of cycles, let us first introduce the instantaneous number of cycles spent near some instantaneous frequency $F$. It is naturally defined by multiplying the integrand in Eq. (2.18) by $F$, considered as the length of an interval $\pm \Delta F= \pm \frac{F}{2}$ around $F$ i.e.

$$
N(F) \equiv \frac{F}{2 \pi} \frac{d \phi}{d F} \equiv \frac{F^{2}}{d F / d t},
$$

where we have used $d \phi / d t \equiv 2 \pi F(t)$. Note that the instantaneous $N$ can be considered either as a function of the running frequency $F(t)$, or, directly, of time.

The instantaneous number of cycles plays an important role both in defining the observability of a signal, and in controlling partially the validity of the stationary phase approximation. The square of the optimal SNR reads

$$
\rho^{2} \equiv\left(\frac{S}{N}\right)^{2}=\int_{-\infty}^{+\infty} d f \frac{|\tilde{h}(f)|^{2}}{S_{n}(f)}
$$

In the stationary phase approximation (discussed at length and improved below; but here we use standard results for orientation) the modulus of the Fourier transform of the real signal $h(t)=2 a(t) \cos \phi(t)$, reads $|\tilde{h}(f)| \simeq a\left(t_{f}\right) / \sqrt{\dot{F}\left(t_{f}\right)}$ 
where $t_{f}$ is the time when the instantaneous frequency $F(t)$ reaches the value $f$. Therefore, the squared modulus can be written as

$$
|\tilde{h}(f)|^{2} \simeq \frac{a^{2}(f)}{d f / d t} \equiv \frac{1}{f^{2}} N(f) a^{2}(f) .
$$

Finally, the SNR can be rewritten as

$$
\rho^{2} \equiv\left(\frac{S}{N}\right)^{2}=\int_{-\infty}^{+\infty} \frac{d f}{f} \frac{N(f) a^{2}(f)}{f S_{n}(f)}=\int_{-\infty}^{+\infty} \frac{d f}{f} \frac{h_{s}^{2}(f)}{h_{n}^{2}(f)}
$$

where we have introduced the notation $h_{s}^{2}(f) \equiv N(f) a^{2}(f)$ and $h_{n}^{2}(f) \equiv f S_{n}(f)$. Here, $h_{n}^{2}(f)$ is the usual squared amplitude of the effective gravitational wave noise at the frequency $f$, i.e. the minimum gravitational wave amplitude detectable in a bandwidth $\pm f / 2$ around frequency $f$. Eq. (2.22) exhibits that the squared amplitude of the corresponding effective gravitational wave signal is $h_{s}^{2}(f) \equiv N(f) a^{2}(f)=f^{2}|\tilde{h}(f)|^{2}$, i.e. that the "bare" amplitude $a(f) \equiv a(t(f))$ is effectively multiplied by $\sqrt{N(f)}[5] 6$. Eq. (2.22) also exhibits the relative weight with which each cycle counts for detectability purposes. Per logarithmic frequency interval this weight is simply

$$
w(f) \equiv a^{2}(f) / h_{n}^{2}(f) .
$$

Therefore it is natural to define the number of useful cycles as

$$
N_{\text {useful }} \equiv\left(\int_{F_{\min }}^{F_{\max }} \frac{d f}{f} w(f) N(f)\right)\left(\int_{F_{\min }}^{F_{\max }} \frac{d f}{f} w(f)\right)^{-1},
$$

where $F_{\min }$ is the low-frequency seismic-cutoff below which $h_{n}^{2}(f)$ is essentially infinite and where the upper-cutoff $F_{\max }$ is the frequency at which the signal itself shuts off. For illustration, we list in Table 1 the number of useful cycles and the total number of cycles for representative systems and orders of approximation. For Newtonian chirps the total number of cycles is

$$
N_{\mathrm{tot}}^{\mathrm{Newt}}=\frac{\left(\pi m f_{\mathrm{s}}\right)^{-5 / 3}-\left(\pi m f_{\max }\right)^{-5 / 3}}{32 \pi \eta}
$$

where $f_{\mathrm{s}}$ is the seismic cutoff. The total number of cycles for relativistic chirps is always smaller than $N_{\mathrm{tot}}^{\text {Newt }}$. From Table 1 it is clear that the number $N_{\text {useful }}$ is usually much smaller than $N_{\text {tot }}$, Eq. (2.18). Note that for massive systems $N_{\text {useful }}$ becomes quite small. The number of useful cycles given in Table thave been computed for the initial LIGO noise curve Eq. (1.4a). The corresponding numbers for the VIRGO noise curve Eq. (1.5a would be larger both because the VIRGO sensitivity curve peaks at a lower frequency, and because it is flatter.

To explore in more detail the case of systems with a small number of useful cycles we display in Fig. 3 (on a linear-log plot) the various factors of the logarithmic integrand on the RHS of Eq. (2.22) for two different binary systems. The instantaneous number of cycles $N(f)$ in the Newtonian approximation is plotted, together with square of the amplitude $a^{2}(f)$, their product the effective gravitational wave amplitude $h_{s}^{2}(f)=N(f) a^{2}(f)$, the reciprocal of effective noise $h_{\mathrm{n}}^{2}(f)=f S_{n}(f)$, (cut off after $F_{\max }=F_{\mathrm{LSO}}$ ) and the power per log bin of the square of the SNR $d \rho^{2} / d(\log f)$. On the left, the scale on the y-axis corresponds to $N(f)$. On the right it corresponds to the amplitude on an arbitrary scale. Other quantities are on an arbitrary scale. The top panel is for a lighter mass binary $\left(m_{1}=1.4 M_{\odot}\right.$, $\left.m_{2}=10 M_{\odot}\right)$ and the bottom panel for a heavier one $\left(m_{1}=m_{2}=10 M_{\odot}\right)$.

The last Figure exhibits two useful lessons which are well-known but are particularly important to keep in mind when reading the present paper. First, because of the mass-scaling of the gravitational wave frequency at the last stable orbit, given in the lowest (Schwarzschild-like) approximation by Eq. 2.14), it is only for systems with total mass $m=m_{1}+m_{2} \gtrsim 13 M_{\odot}$ that the peak of the SNR logarithmic frequency-distribution $f_{p}$ becomes comparable,

\footnotetext{
${ }^{5}$ In the following, it will be necessary to distinguish carefully between the instantaneous frequency $F$ and the Fourier variable $f$. In the present Section this distinction is not very important and we shall freely change notation $f \leftrightarrow F$. Similarly, the gravitational wave flux and factored flux functions which following standard notation was denoted by $F(v)$ and $f(v)$ in 13 are denoted by $\mathcal{F}(v)$ and $l(v)$ in this paper to avoid confusion with the instantaneous gravitational wave frequency and Fourier variable respectively.
} 
within a factor of two, to $F_{\max }=F_{\mathrm{LSO}}$. This statement critically depends on the characteristic frequency entering the considered noise-curve. For instance, in Fig. 3 we have used the initial LIGO curve Eq. (1.4a) for which $f_{p}=0.825 f_{0}=165 \mathrm{~Hz}$. Note that the peak of the logarithmic SNR integrand, $f_{p}$, is very close to the minimum of the effective noise amplitude $h_{n}^{2}(f)=f S_{n}(f)$, which is located (as mentioned above) at $f_{\text {det }}=0.8347 f_{0} \simeq 167$ Hz. This is because, the frequency dependence of the factor $N(f) \propto\left(\eta v^{5}\right)^{-1} \propto f^{-5 / 3}$ (which in the effective signal $h_{s}^{2}(f)=N(f) a^{2}(f)$, favours lower frequencies) is nearly compensated by the frequency dependence of the bare amplitude $a^{2}(f) \propto v^{4} \propto f^{4 / 3}$ (which favours higher frequencies).

A second lesson, to be drawn from Fig. 3 is that the number of useful cycles also becomes small in the same problematic case of massive systems. To see this more clearly, let us write down the explicit expression for the instantaneous number of cycles. In the Newtonian case (for which the basic formulas are recalled in Sec. III A below), one has

$$
N_{\text {Newtonian }}(f)=\frac{5}{24 \pi} \frac{1}{4 \eta} \frac{1}{v^{5}},
$$

where $v=(\pi m f)^{1 / 3}$. The lowest value of $N$ is physically that formally reached at the upper frequency-cutoff $f=F_{\max }=F_{\mathrm{LSO}}$. For $v_{\mathrm{LSO}}=1 / \sqrt{6}$ (the "Schwarzschild" value), the above equation reads

$$
N_{\text {Newtonian }}\left(F_{\mathrm{LSO}}\right)=\frac{5}{24 \pi} 6^{5 / 2} \frac{1}{4 \eta} \simeq \frac{5.8477}{4 \eta},
$$

where we recall that $\eta \leq 1 / 4$ and that the upper value $\eta_{\max }=1 / 4$ is reached for equal mass systems $m_{1}=m_{2}$. Therefore for comparable-mass, massive systems, the Newtonian approximation suggests that the useful number of cycles will be rather small $(\sim 6)$ and concentrated near the LSO. As we shall see later, if we were interested in estimating the Fourier transform (FT) of an analytic Newtonian-like signal, even such a smallish number of cycles (and even a smaller one, down to $N \sim 1$ ) would be enough to ensure that the leading correction to the stationary phase approximation is small. However, the complication comes from the combination of two facts: (i) the signal essentially terminates at the LSO crossing time $t_{\mathrm{LSO}}$, and (ii) one crucially needs a relativistic description of the evolution near the LSO. Using the formulas and the notation of Sec. IV below, we find that the relativistic prediction for the instantaneous number of cycles (in the adiabatic inspiral approximation) is

$$
N_{\text {relat }}(f)=-\frac{1}{3 \pi} v^{4} \frac{E^{\prime}(v)}{\mathcal{F}(v)} .
$$

By definition of the LSO (see e.g. the discussion in DIS) the derivative $E^{\prime}(v)$ vanishes at $v=v_{\text {LSO }}$. Therefore, the instantaneous number of cycles is smaller in the relativistic case than in the Newtonian one and actually tends to zero near the LSO. We shall tackle in Sec. IV below the problem that this vanishing of $N\left(F_{\mathrm{LSO}}\right)$ causes for the stationary phase approximation. In this introductory Section let us only illustrate the problem by plotting the Newtonian and relativistic values of $N(F(t))$. In Fig. 4 we plot the instantaneous number of cycles for the Newtonian and second post-Newtonian $P$ - approximant waveforms in the last few cycles of the binary inspiral for a $\left(20 M_{\odot}, 20 M_{\odot}\right)$ system. We also show the development of the waveform in this interval on an arbitrary scale. These plots demonstrate how the number of useful cycles diminishes as one gets close to the LSO and lead us to anticipate the subtleties in the detectability of signals whose LSO is near the most sensitive part of the frequency response of the detector.

\section{Loss of SNR due to edge effects}

As we already mentioned, in addition to the problem of a vanishing instantaneous number of cycles near the LSO in the relativistic case, the main problem with the acccuracy of the stationary phase approximation comes from the fact that the Fourier transform of a time-windowed signal $\tilde{h}_{\mathrm{tw}}(f)=\mathrm{FT}\left[h_{\mathrm{tw}}(t)\right]$ differs from the frequency-windowed SPA $\tilde{h}_{\text {spaw }}(f)=\theta\left(F_{\max }-f\right) \tilde{h}_{\text {spa }}(f)$ because of some 'edge effects' in the frequency-domain, linked to the abrupt termination of the signal in the time-domain. These edge-effects comprise some additional oscillatory behaviour in $\tilde{h}(f)$ for $f<F_{\mathrm{LSO}}$, as well as a decaying oscillatory tail in the usually disregarded frequency-domain for $f>F_{\mathrm{LSO}}$.

Let us here anticipate on the results below and use a first-order approximation to discuss the main features of the corrections brought by the time-windowing. Roughly (see below) the exact Fourier transform can be written as

$$
\tilde{h}_{X}(f) \simeq \tilde{h}_{\mathrm{win}}^{\mathrm{spa}}(f)+\varepsilon(f),
$$

where $\tilde{h}_{\mathrm{win}}^{\mathrm{spa}}(f)=\tilde{h}^{\mathrm{spa}}(f) \theta\left(F_{\max }-f\right)$ is the usually considered frequency-windowed SPA. The difference $\varepsilon(f)$ is approximately of the form 


$$
\begin{aligned}
\varepsilon(f) & \simeq \mathcal{D}(f) \tilde{h}^{\text {spa }}(f), \\
\text { where, } \quad \mathcal{D}(f) & \equiv \mathcal{C}(f)-\theta\left(F_{\max }-f\right),
\end{aligned}
$$

with the correction factor $\mathcal{C}(f)$ given by Eq. (3.23) below (with any choice of $\zeta(f)$ in the present approximation) and where $\tilde{h}^{\text {spa }}(f)$ is some smooth continuation of $\tilde{h}_{\text {win }}^{\text {spa }}(f)$ from the domain $f<F_{\text {LSO }}$ to the domain $f>F_{\mathrm{LSO}}$. (For the present purpose one can think that $\tilde{h}^{\text {spa }}(f)$ is simply given by the Newtonian approximation).

Starting from Eq. (2.29) one can compute the overlap between the exact $\tilde{h}(f)$ and the usually considered frequencywindowed SPA $\tilde{h}_{\text {win }}^{\text {spa }}(f)$

$$
\mathcal{O}=\frac{\left\langle h_{X}, h_{\text {win }}^{\mathrm{spa}}\right\rangle}{\sqrt{\left\langle h_{X}, h_{X}\right\rangle\left\langle h_{\mathrm{win}}^{\mathrm{spa}}, h_{\mathrm{win}}^{\mathrm{spa}}\right\rangle}} .
$$

As recalled above [see Eq. (2.7)] this overlap, if it is significantly smaller than one, represents a loss in SNR. To lowest order in $\varepsilon$ the overlap Eq. (2.32) differs from 1 by

$$
1-\mathcal{O} \simeq \frac{1}{2\left\|h_{X}\right\|^{2}}\left(\|\varepsilon\|^{2}-\left|\left\langle\varepsilon, \hat{h}_{X}\right\rangle\right|^{2}\right),
$$

where $\|\varepsilon\|^{2} \equiv\langle\varepsilon, \varepsilon\rangle$, and $\hat{h}_{X} \equiv h_{X} /\left\|h_{X}\right\|$. In inserting the explicit result Eqs.(2.30) and (2.31) for $\varepsilon$ one sees that the second term on the RHS of Eq. (2.33) is much smaller than the first (because the oscillations in $\mathcal{D}(f)$ are integrated against the smooth variation of $\left.\tilde{h}^{\text {spa }}(f)\right)$. Finally, if we define the weight function

$$
\sigma(f) \equiv \frac{f\left|\tilde{h}^{\mathrm{spa}}(f)\right|^{2}}{S_{n}(f)} \simeq \frac{N(f) a^{2}(f)}{h_{n}^{2}(f)}=\frac{h_{s}^{2}(f)}{h_{n}^{2}(f)},
$$

which is the full logarithmic weight function appearing in the squared SNR, Eq. (2.22), we can write

$$
1-\mathcal{O} \simeq \frac{1}{2} \int_{0}^{\infty} \frac{d f}{f} \sigma(f)|\mathcal{D}(f)|^{2}\left(\int_{0}^{F_{\max }} \frac{d f}{f} \sigma(f)\right)^{-1} .
$$

As will be discussed later (see Fig. [D) the function $\mathcal{D}(f)=\mathcal{C}(f)-\theta\left(F_{\max }-f\right)$ is concentrated in an interval of order $\sqrt{\dot{F}\left(t_{\max }\right)}$ around $f \simeq F_{\max }$ and decays on both sides [like $\left.1 / \zeta(f) \propto 1 /\left(f-F_{\max }\right)\right]$ when $f$ gets away from $F_{\max }$. The total integral of $|\mathcal{D}(f)|^{2}$ is finite and of order unity. Thus, we see from Eq. (2.35) that when the characteristic frequency $f_{\mathrm{p}}$ around which $\sigma(f)$ is concentrated satisfies $f_{\mathrm{p}} \ll F_{\max }$ we shall have a rough estimate

$$
1-\mathcal{O} \sim \frac{\sigma\left(F_{\max }\right)}{\sigma\left(f_{\mathrm{p}}\right)} \frac{\sqrt{\dot{F}\left(t_{\max }\right)}}{F_{\max }}=\frac{\sigma\left(F_{\max }\right)}{\sigma\left(f_{\mathrm{p}}\right)} \frac{1}{\sqrt{N\left(F_{\max }\right)}},
$$

while in the opposite limit where $f_{\mathrm{p}} \gg F_{\max }$, we get the rough estimate

$$
1-\mathcal{O} \sim \frac{\sqrt{\dot{F}\left(t_{\max }\right)}}{F_{\max }}=\frac{1}{\sqrt{N\left(F_{\max }\right)}} .
$$

In the case where $f_{\mathrm{p}} \ll F_{\max }$ the factor $\sigma\left(F_{\max }\right) / \sigma\left(f_{\mathrm{p}}\right)$ in the RHS of Eq. (2.36) is very small. Therefore, even if the number of cycles $N\left(F_{\max }\right)$ is not very large, Eq. (2.36) predicts that a simple frequency-windowed SPA will have excellent overlap with the exact $\tilde{h}(f)$. On the other hand, Eq. (2.37) shows that in the reverse limit $f_{\mathrm{p}} \gg F_{\max }$ which means in fact when $f_{\mathrm{p}} \gtrsim F_{\max }$, the overlap will become bad if $\sqrt{N\left(F_{\max }\right)}$ is not very large. As we have seen that $\sqrt{N\left(F_{\max }\right)}$ gets as low as $\sqrt{5.85} \simeq 2.4$ in the Newtonian case, and reaches smaller values in the relativistic case, we expect that the cases where the frequency-windowed SPA has a bad overlap with $\tilde{h}(f)$ are those where $f_{\mathrm{p}}$ becomes comparable, say within a factor of two, to $F_{\max }=F_{\mathrm{LSO}}$. We recover the same conclusion as above, which was the conclusion already pointed out in DIS: namely the signal from massive systems $\left[m \gtrsim 13 M_{\odot}\right.$ if $f_{\mathrm{p}}=165 \mathrm{~Hz}$, corresponding to $f_{0}=200 \mathrm{~Hz}$, more generally, $\left.m \gtrsim 13\left(165 \mathrm{~Hz} / f_{\mathrm{p}}\right) M_{\odot}\right]$, when treated (as they should be) relativistically will be badly represented by the usual frequency-windowed SPA. This conclusion obtained from an analytical approximation is borne out by the numerical computations shown in Fig. 目 above (see also Table II below). It is mainly for such systems that the work presented in the following Sections will be mandatory. But even for lower mass systems, we shall construct here for the first time the Fourier-domain version of the (time-domain) $\mathrm{P}$-approximants introduced in DIS. Since P-approximants provide better templates than the usually considered Tapproximants [13], the work of this paper will be useful for all types of systems, even the less massive ones. 


\section{Fourier transform of time-truncated chirps}

To introduce the detailed analysis that we shall give in the following Sections, let us start by delineating some general mathematical facts about the integrals we have to deal with. We will be interested in evaluating the Fourier-transform $\tilde{h}(f)$ of a time-truncated chirp $h(t)=2 a(t) \cos \phi(t) \theta\left(t_{\max }-t\right)$. After decomposing the cosine into complex exponentials, the Fourier integral leads to a sum of two integrals of the form $\int_{-\infty}^{t_{\max }} d t a(t) e^{i \psi_{f}^{ \pm}(t)}$ with phases $\psi_{f}^{ \pm}=2 \pi f t \pm \phi(t)$. Let us then, for generality, discuss the properties of integrals of the type

$$
I(\varepsilon)=\int_{t_{a}}^{t_{b}} d t a(t) e^{i \frac{\psi(t)}{\varepsilon}} .
$$

Here, we have introduced a formal 'small parameter' $\varepsilon$ (set to unity at the end of the calculation) to formalize the fast variation of the phase compared to that of the amplitude.

Let us first note that: (i) if the phase has no stationary point $\dot{\psi}(t) \neq 0$ for $t \in\left[t_{a}, t_{b}\right]$, (ii) if the amplitude vanishes smoothly at the edge points $t_{a}$ and $t_{b}$, which might be pushed to $\pm \infty$ and (iii) if the functions $a(t)$ and $\psi(t)$ are smooth $\left(\mathcal{C}^{\infty}\right)$ within the interval $\left[t_{a}, t_{b}\right]$, the integral $I(\varepsilon)$ tends to zero with $\varepsilon$, faster than any power. This can be seen by integrating by parts. To simplify the calculation we can [thanks to the assumption (i)] use $\psi$ as integration variable. This yields

$$
I=\int_{\psi_{a}}^{\psi_{b}} d \psi A(\psi) e^{i \psi}
$$

where $A(\psi)=[a(t) / \dot{\psi}(t)]_{t(\psi)}$ where $t(\psi)$ denotes the (unique) solution in $t$ of $\psi=\psi(t)$ and where $\psi_{a}=\psi\left(t_{a}\right), \psi_{b}=$ $\psi\left(t_{b}\right)$. Using $e^{i \psi / \varepsilon}=\frac{\varepsilon}{i} \frac{d}{d \psi}\left(e^{i \psi / \varepsilon}\right)$, integrating by parts, and using [thanks to assumption (ii)] the vanishing of $A(\psi)$ at the edges, leads to

$$
I(\varepsilon)=i \varepsilon \int_{\psi_{a}}^{\psi_{b}} d \psi A^{\prime}(\psi) e^{\frac{i \psi}{\varepsilon}} .
$$

Using [assumption (iii)] the vanishing of all the derivatives of $A(\psi)$ at the edges, we can iterate the result Eq. (2.40) to any order:

$$
I(\varepsilon)=(i \varepsilon)^{n} \int_{\psi_{a}}^{\psi_{b}} d \psi A^{(n)}(\psi) e^{\frac{i \psi}{\varepsilon}} .
$$

The result Eq. 2.41) means that, when $\varepsilon \rightarrow 0, I(\varepsilon)=\mathcal{O}\left(\varepsilon^{n}\right)$ for any integer $n$, i.e. $I(\varepsilon)$ vanishes faster than any power of $\varepsilon$. It does not mean that $I(\varepsilon)$ is zero for any finite (but small) value of $\varepsilon$. For instance, under stronger assumptions about the existence and properties of an analytic continuation of the function $A(\psi)$ in the complex $\psi$ plane it follows that $I(\varepsilon) \sim A e^{-\frac{B}{\varepsilon}}$ for some constants $A$ and $B$. For reasonably small values of $\varepsilon$ such exponentially small contributions are numerically negligible. [We shall see later that the 'small parameter' $\varepsilon$ (or better $\varepsilon / B$ ) is typically of order $1 /(2 \pi N)$ where $N \equiv F^{2} / \dot{F}$, is the instantaneous number of cycles.]

We conclude, therefore that the integral $I$ will be (in most relevant cases) numerically non-negligible only if the assumptions above are violated. In other words, the value of $I(\varepsilon)$ will be dominated by the contributions coming from either (i) from stationary-phase points, $\dot{\psi}\left(t_{s}\right)=0$, or (ii) from the edge points $t_{a}$ and/or $t_{b}$. Let us (for simplicity) assume that there is (at most) one stationary-phase point $t_{s}$, and that it is of the normal parabolic type, i.e. $\psi(t)=\psi_{s}+\frac{1}{2 !} \ddot{\psi}_{s}\left(t-t_{s}\right)^{2}+\mathcal{O}\left(\left(t-t_{s}\right)^{3}\right)$ with $\ddot{\psi}_{s} \neq 0$. Let us also assume that $a\left(t_{a}\right) \neq 0, a\left(t_{b}\right) \neq 0$. $[$ We maintain here for the moment, the assumption (iii) above about the regularity of the functions $a(t)$, $\psi(t)$ in the closed interval $\left.\left[t_{a}, t_{b}\right].\right]$ Then, assuming analyticity of the involved functions, the mathematically most rigorous way of decomposing $I$ as the sum (modulo nonperturbative small contributions of the type discussed above) of a stationarypoint contribution $I_{\text {stationary }}$ and of edge contributions $I_{\text {edge }}=I_{\text {edge }}^{a}+I_{\text {edge }}^{b}$ is to deform the original (real) contour of integration into the complex plane [45,31. The deformed contour must be such that near $t_{s}$ it leads to a basic integral of the type $\int_{-\alpha}^{\beta} d x e^{-b x^{2}}\left[c_{0}+c_{1} x+c_{2} x^{2}+\cdots\right]$, while near each end point it leads to integrals of the type $\int_{0}^{\gamma} d y e^{-c y}\left[d_{0}+d_{1} y+d_{2} y^{2}+\cdots\right]$. It is then easy to find the structure of the expansion of both $I_{\text {stationary }}$ and $I_{\text {edge }}$ in powers of $\varepsilon$, as $\varepsilon \rightarrow 0$. For instance, it is convenient near $t_{s}$ to introduce a scaled variable: $t-t_{s}=\varepsilon^{\frac{1}{2}} \tau$ (before rotating $\tau$ to complex values $\tau=e^{ \pm \frac{i \pi}{4}} x$ ), so that the phase scales as 


$$
\frac{\psi}{\varepsilon}=\frac{\psi_{s}}{\varepsilon}+\frac{1}{2 !} \ddot{\psi}_{s} \tau^{2}+\frac{\sqrt{\varepsilon}}{3 !} \psi_{s}^{(3)} \tau^{3}+\cdots
$$

where $\psi_{s}^{(3)} \equiv d^{3} \psi_{s} / d t^{3}$. Expanding then the integrand of this $\tau$-integral in powers of $\varepsilon^{\frac{1}{2}}$ leads to an integral of the symbolic type

$$
I_{\text {stationary }} \sim \varepsilon^{\frac{1}{2}} \int d x e^{-x^{2}}\left[1+\varepsilon^{\frac{1}{2}} x^{\text {odd }}+\varepsilon x^{\text {even }}+\varepsilon^{\frac{3}{2}} x^{\text {odd }}+\cdots\right]
$$

where $x^{\text {odd }}\left(x^{\text {even }}\right)$ denotes a sum of terms $\sim x^{2 k+1}\left(x^{2 k}\right)$. This yields (using the fact that the terms $\int_{-\alpha / \sqrt{\varepsilon}}^{\beta / \sqrt{\varepsilon}} d x e^{-x^{2}} x^{\text {odd }}$ are exponentially small) an expansion of the type

$$
I_{\text {stationary }} \sim \varepsilon^{\frac{1}{2}}\left[C_{0}+C_{1} \varepsilon+C_{2} \varepsilon^{2}+\cdots\right]
$$

The structure of the 'edge' contribution $I_{\text {edge }}$ can be obtained by a similar technique. Now the appropriate scaling is different, e.g. $t-t_{a}=\varepsilon \tau$, and one ends up with integrals of the type $\int_{0}^{\gamma / \varepsilon} d y e^{-y} y^{n}$. This yields,

$$
I_{\text {edge }} \sim \varepsilon\left[D_{0}+D_{1} \varepsilon+D_{2} \varepsilon^{2} \ldots\right] .
$$

The aim of this preliminary discussion was to point out the structures Eqs. (2.44) and (2.45) of the two main contributions to a generic oscillatory integral of the form Eq. (2.38). Note that while the leading contribution is given by the lowest order term in the stationary-point or saddle-point expansion Eq. (2.44), the next-to-leading contribution comes from the lowest order edge-correction Eq. (2.45). One generally expects that $I_{\text {edge }}$ will be only

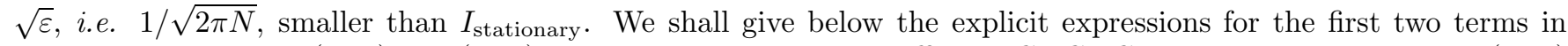
both expansions Eqs. (2.44) and (2.45). We shall see that each coefficient $C_{0}, C_{1}, C_{2} \cdots \cdots D_{0}, D_{1}, \cdots$ in Eqs. (2.44) and (2.45) is a combination of derivatives (of increasing total order) of $a(t)$ and $\psi(t)$ evaluated at $t_{s}$ for Eq. (2.44) and at $t_{a}$ or $t_{b}$ for Eq. (2.45). We note in advance that, for actual calculations, the simplest way to evaluate the explicit forms of the expansions Eqs. (2.44) and (2.45) is not necessarily to follow the complex-contour route sketched above. In the case of Eq. (2.44) one can deal directly with the original stationary-point-expanded integral written as $\propto \varepsilon^{\frac{1}{2}} \int d \tau e^{i \ddot{\psi}_{s} \tau^{2}}\left[\tau_{0}+c_{1} \tau+\cdots\right]$, and in the case of Eq. (2.45) the simplest is to keep the boundary terms in the integration-by-parts approach Eqs. (2.40) and (2.41) given above for the simple case where they were neither stationary phase points, nor boundary contributions.

To put in context the analysis that we shall perform below, let us finally mention two serious limitations of the assumptions leading to Eqs. 2.44) and (2.45). First, in the analysis above, based on the introduction of the formal parameter $\varepsilon \rightarrow 0$, we have assumed that the stationary-phase point $t_{s}$ was parametrically separated from the edges $t_{a}$ or $t_{b}$, i.e. that $\left|t_{s}-t_{a}\right|$ and $\left|t_{s}-t_{b}\right|$ were much larger than the characteristic Gaussian width $\Delta t=\sqrt{\varepsilon / \ddot{\psi_{s}}}=\mathcal{O}(\sqrt{\varepsilon})$ associated to the stationary point. As we shall see, this limitation is unacceptable for the application we have in mind and we shall have to introduce new tools to overcome it. A second limitation (which compounds with the first and will lead to an unavoidable complexity of our treatment) is the seemingly innocent assumption (iii) above, namely the hypothesis that the functions $a(t)$ and $\psi(t)$ are infinitely differentiable within the closed interval $\left[t_{a}, t_{b}\right](i . e$. including also the end points). As we shall see, in the physically relevant case of a relativistic (adiabatic) chirp the functions $a(t)$ and $\psi(t)$ will not be $\mathcal{C}^{\infty}$ at the physically imposed upper cutoff $t_{b}=t_{\mathrm{LSO}}$. This will require us to introduce new types of expansions and new tools to deal with the relativistic edge contribution in addition to the modification of the stationary-phase approximation needed in the case where $t_{s}$ is near the edge, in the sense that $\left|t_{s}-t_{b}\right|=\mathcal{O}(\sqrt{\varepsilon})$.

\section{IMPROVED STATIONARY PHASE APPROXIMATION FOR TIME-WINDOWED NEWTONIAN-LIKE SIGNALS}

\section{A. The usual stationary phase approximation}

Let us begin by a quick recall of the usual treatment of the stationary phase approximation to a chirp. Consider a signal,

$$
\begin{aligned}
h(t) & =2 a(t) \cos \phi(t)=a(t) e^{-i \phi(t)}+a(t) e^{i \phi(t)}, \\
\text { where, } \frac{d \phi(t)}{d t} & \equiv 2 \pi F(t)>0 .
\end{aligned}
$$


We shall say that a signal is "Newtonian-like" if the instantaneous frequency $F(t)$, defined by Eq. (3.1b), increases without limit when $t$ runs over its full (mathematically allowed) range. (Note that we conventionally consider only positive instantaneous frequencies.) For instance, at the Newtonian order, the explicit forms for the chirp amplitude, phase and frequency of the gravitational waves are respectively given by:

$$
\begin{aligned}
a(t) & =\mathcal{C}_{\mathcal{M}}(\pi \mathcal{M} F(t))^{2 / 3}, \\
\phi(t) & =\phi_{c}-2\left[\frac{\left(t_{c}-t\right)}{5 \mathcal{M}}\right]^{5 / 8}, \\
\pi \mathcal{M} F(t) & =\left[\frac{5 \mathcal{M}}{256\left(t_{c}-t\right)}\right]^{3 / 8},
\end{aligned}
$$

where $\mathcal{M}$ is the chirp mass given by $\mathcal{M}=\eta^{3 / 5} m$ in terms of the total mass $m$ and the symmetric mass ratio $\eta$; $\phi_{c}$ the gravitational wave phase at instant of coalescence $t_{c}$ and $\mathcal{C}_{\mathcal{M}}$ is the product of a function of different angles, characterising the relative orientations of the binary and the detector, with the ratio $\mathcal{M} / d$ where $d$ is the distance to the source (see below). Note that the function $F(t)$ increases without limit as $t$ tends to the formal coalescence time $t_{c}$.

Coming back to a general signal, the Fourier transform is defined by Eq. (1.1). Because the signal $h(t)$ is real, we have the identity $\tilde{h}(-f) \equiv(\tilde{h}(f))^{*}$. It therefore suffices to compute the Fourier transform for positive values of the frequency $f$. [Note that we use a lower case letter to distinguish the Fourier variable $f$ from the instantaneous frequency $F(t)$.] The Fourier transform of a generic signal of the form Eq. (3.1a) reads

$$
\begin{aligned}
\tilde{h}(f) & =\tilde{h}_{-}(f)+\tilde{h}_{+}(f), \\
\text { where, } \tilde{h}_{-}(f) & \equiv \int_{-\infty}^{\infty} d t a(t) e^{i(2 \pi f t-\phi(t))}, \\
\tilde{h}_{+}(f) & \equiv \int_{-\infty}^{\infty} d t a(t) e^{i(2 \pi f t+\phi(t))} .
\end{aligned}
$$

The integrands of $\tilde{h}_{ \pm}(f)$ are violently oscillating and thus their dominant contributions come from the vicinity of the stationary points of their phase (when such points exist). When $f>0$ (which we shall henceforth assume), only the $\tilde{h}_{-}(f)$ term has such a saddle-point. Therefore, we can write the approximation

$$
\begin{aligned}
\tilde{h}(f) & \simeq \tilde{h}_{-}(f) \simeq \int_{-\infty}^{\infty} d t a(t) e^{i \psi_{f}(t)}, \\
\text { where, } \quad \psi_{f}(t) & \equiv 2 \pi f t-\phi(t) .
\end{aligned}
$$

The saddle-point of the phase $\psi_{f}(t)$ is the value, say $t_{f}$, of the time variable $t$ where $d \psi_{f}(t) / d t=0$, i.e. it is the solution of the equation $F\left(t_{f}\right)=f$. The dominant contribution to the integral Eq. (3.4a) now comes from a time interval near $t=t_{f}$. When the second time derivative of the phase at the saddle-point does not vanish, i.e. when $\dot{F}\left(t_{f}\right) \neq 0$, one can estimate Eq. (3.4a) by replacing $\psi_{f}(t)$ [and $a(t)$ ] by truncated Taylor expansions near $t=t_{f}$, namely

$$
\begin{aligned}
\psi_{f}(t) & \simeq \psi_{f}\left(t_{f}\right)-\pi \dot{F}\left(t_{f}\right)\left(t-t_{f}\right)^{2}, \\
a(t) & \simeq a\left(t_{f}\right) .
\end{aligned}
$$

(The zeroth-order term in Eq. (3.5b) is enough because the first order term $\dot{a}\left(t_{f}\right)\left(t-t_{f}\right)$ vanishes after integration over $t$ ). This leads to a Gaussian integral

$$
\tilde{h}(f) \simeq \int_{-\infty}^{\infty} d t a\left(t_{f}\right) e^{i \psi_{f}\left(t_{f}\right)-i \pi \dot{F}\left(t_{f}\right)\left(t-t_{f}\right)^{2}}
$$

Evaluating this Gaussian integral, one finally obtains the well-known expression for the usual SPA (hereafter abbreviated as uSPA):

$$
\tilde{h}^{\mathrm{uspa}}(f)=\frac{a\left(t_{f}\right)}{\sqrt{\dot{F}\left(t_{f}\right)}} e^{i\left[\psi_{f}\left(t_{f}\right)-\pi / 4\right]}
$$


where $\psi_{f}\left(t_{f}\right)$ is the value of $\psi_{f}(t)$ at $t=t_{f}$.

The conditions for the validity of the SPA are usually assumed to be $\varepsilon_{1} \ll 1$, $\varepsilon_{2} \ll 1$, where

$$
\varepsilon_{1} \equiv\left|\frac{\dot{a}(t)}{a(t) \dot{\phi}(t)}\right| ; \varepsilon_{2} \equiv\left|\frac{\ddot{\phi}(t)}{\dot{\phi}^{2}(t)}\right|=\left|\frac{1}{2 \pi} \frac{\dot{F}(t)}{F^{2}(t)}\right|=\frac{1}{2 \pi N} .
$$

One can assess in a more precise quantitative manner the accuracy of the SPA by computing the leading correction to the integral Eq. (3.6). This leading correction will be given by keeping more terms in the Taylor expansions Eqs. (3.5a) and (3.5b). To keep track of what one means by the 'next order term' in the SPA expansion it is convenient (as in Section IIID above) to formalize the fast variation of the phases $\phi(t)$ and $\psi(t)$ by considering an integral of the form $I=\int d t a(t) \exp (i \psi(t) / \varepsilon)$ with a 'small' parameter $\varepsilon$ (set to unity at the end of the calculation). It is then easy to see [e.g. after the introduction of a rescaled time-variable: $t-t_{f}=\varepsilon^{1 / 2} \tau$ where $t_{f}$ denotes, as above, the saddle point of the phase $\psi_{f}(t)$ ] that the leading correction to the result Eq. (3.7) will be of fractional order $\varepsilon$ [as exhibited in Eq. (2.44)] and will come from keeping two more terms in both the Taylor expansions Eqs. (3.5a) and (3.5b). Expanding in powers of $\varepsilon^{1 / 2}$ leads to integrals of the form $\int_{-\infty}^{\infty} d \tau \tau^{n} \exp \left(-i \pi \dot{F}\left(t_{f}\right) \tau^{2}\right)$ with $n \leq 6$. Finally, one finds that the sum of the usual SPA and of its leading correction is equivalent to multiplying Eq. (3.7) by the correcting phase factor $e^{i \delta}$ where $\left(F^{(3)}\right.$ denoting $\left.d^{3} F / d t^{3}\right)$

$$
\delta=\frac{1}{2 \pi \dot{F}\left(t_{f}\right)}\left[-\frac{1}{2} \frac{\ddot{a}}{a}+\frac{1}{2} \frac{\dot{a}}{a} \frac{\ddot{F}}{\dot{F}}+\frac{1}{8} \frac{F^{(3)}}{\dot{F}}-\frac{5}{24}\left(\frac{\ddot{F}}{\dot{F}}\right)^{2}\right]_{t=t_{f}} .
$$

Therefore, a quantitatively precise criterion for the local validity of the SPA is $\varepsilon_{\text {loc }} \equiv|\delta| \ll 1$. In the case of power-law chirps, $\varepsilon_{\text {loc }}$ is equal to one-fifth of the criterion explicitly given in the recent study [30] of the validity of the SPA. In the case of Newtonian chirps, Eq. (3.9) yields

$$
\delta=\frac{23}{24}\left(\frac{1}{9 \pi} \frac{\dot{F}}{F^{2}}\right)=\frac{23}{24}\left(\frac{1}{9 \pi N}\right) .
$$

Written in terms of $v=(\pi m F)^{1 / 3}$ this reads $\delta=(92 / 45) \eta v^{5}$ which agrees with the corresponding result in [31]. It is interesting to note that Eq. (3.10) formally predicts, at the LSO, $\delta_{L S O}=(4 \eta) \times 0.58 \%$ which is quite small. Alternatively, one can say that Eq. (3.10) predicts that even if the instantaneous number of cycles were as small as $N \sim 1$, the local correction to the SPA would be small $(\delta=0.0339 / N)$. This result does not mean, however, that we can use the usual SPA Eq. (3.7) to estimate with sufficient accuracy the FT of a real inspiral signal. Indeed, even if we were considering a Newtonian-like signal (where $N$ stays away from zero at the LSO) the correcting phase factor Eq. (3.9) represents just the local correction to the SPA, i.e. the correction due to higher-terms in the local expansion near the saddle-point. There are also global corrections to the SPA coming from the entire integration domain, and, most importantly (as emphasized in [30]) from the end-points of the time-integration. In addition, there is also a correction coming from the neglected contribution $\tilde{h}_{+}(f)$ in Eq. (3.3b $)$. Before considering them in detail, let us also note that Eq. (3.9) indicates that $\delta$ blows up to infinity, at the LSO, in the case of a relativistic GW chirp (because $F(t) \sim F_{\mathrm{LSO}}-a\left(t_{\mathrm{LSO}}-t\right)^{1 / 2}$ there; see below). This shows again that, independently of the problems linked to the time-windowing, relativistic signals will pose special difficulties. But let us start by studying the simpler case of time-truncated Newtonian-like signals, by which we mean that $N \equiv F^{2} / \dot{F}$ stays away from zero at the upper time cut-off.

\section{B. Beyond the usual stationary phase approximation}

We therefore consider time-domain signals of the form

$$
h(t)=2 a(t) \cos \phi(t) \theta\left(t_{\max }-t\right),
$$

where $\theta$ denotes the Heaviside step function, $(\theta(x)=1$, for $x>0$ and $\theta(x)=0$, for $x<0)$. This time-windowing has three effects: (i) it induces oscillations in the usually considered frequency-domain $f<F_{\max }$, (ii) it generates a tail in the usually disregarded frequency-domain $f>F_{\max }$, and (iii) it renders non-negligible the 'non-resonant' contribution $\tilde{h}_{+}(f)$. Here, $F_{\max }$ denotes the instantaneous gravitational wave frequency reached at $t=t_{\max }$, i.e. $F_{\max } \equiv F\left(t_{\max }\right)$. The main purpose of the present paper is to model and estimate, analytically, as accurately as possible all these 
effects. The case where the saddle-point $t_{f}$ is (below and) far away from the upper-cutoff $t_{\text {max }}$ has been recently considered in [31]. However, this case is not the physically relevant one. As pointed out in DIS, the case where the usual SPA becomes unacceptably inaccurate is the case of massive systems for which the signal emits not many cycles in the detector's bandwidth before crossing the last stable orbit. In this case the most important frequencies are located around the effective-cutoff frequency $F_{\max } \sim F_{\mathrm{LSO}}$ (and as we shall see below, it is important to estimate the Fourier transform accurately, both for $f<F_{\max }$ and for $\left.f>F_{\max }\right)$. Here, we shall provide an approximate analytical treatment valid in this crucial range of frequencies.

Let us first state clearly our notation. We decompose the FT of the time-windowed signal Eq. (3.11) as

$$
\begin{aligned}
\tilde{h}(f) & =\tilde{h}_{-}(f)+\tilde{h}_{+}(f), \\
\text { where, } \tilde{h}_{-}(f) & =\int_{-\infty}^{t_{\max }} d t a(t) e^{i \psi_{f}^{-}(t)}, \\
\tilde{h}_{+}(f) & =\int_{-\infty}^{t_{\max }} d t a(t) e^{i \psi_{f}^{+}(t)}, \\
\psi_{f}^{-}(t) \equiv \psi_{f}(t) & \equiv 2 \pi f t-\phi(t), \\
\psi_{f}^{+}(t) & \equiv 2 \pi f t+\phi(t) .
\end{aligned}
$$

We shall refer to the contribution $\tilde{h}_{-}(f)$ as the 'resonant' contribution because the equation defining the saddle point it contains, $F\left(t_{f}\right)=f$, corresponds to a resonance between the Fourier-frequency $f$ and the instantaneous gravitational wave frequency $F(t)$.

\section{Edge contribution to the non-resonant integral $\tilde{h}_{+}(f)$}

Before dealing with the oscillatory and tail corrections to the resonant contribution $\tilde{h}_{-}(f)$ of $\tilde{h}(f)$, we shall first deal with the non-resonant contribution $\tilde{h}_{+}(f)$. When $f>0$, the phase $\psi_{f}^{+}(t)$ in contrast to $\psi_{f}^{-}(t)$ has no stationary point. Let us therefore consider the general problem of approximating an integral of the form

$$
I=\int_{t_{a}}^{t_{b}} d t a(t) e^{i \psi(t)}
$$

where $\psi(t)$ is always monotonically varying $(\dot{\psi} \neq 0)$. We can then use $\psi$ as integration variable. This yields, as above,

$$
I=\int_{\psi_{a}}^{\psi_{b}} d \psi A(\psi) e^{i \psi}
$$

where $A(\psi)=[a(t) / \dot{\psi}(t)]_{t(\psi)}$ where $t(\psi)$ denotes the (unique) solution in $t$ of $\psi=\psi(t)$ and where $\psi_{a}=\psi\left(t_{a}\right), \psi_{b}=$ $\psi\left(t_{b}\right)$. One can treat $\psi$ as a fast varying phase, so that by comparison, the amplitude $A(\psi)$ varies slowly when $\psi$ varies by $2 \pi$ (as above this could be formalized by the formal replacement $\psi \rightarrow \psi / \varepsilon$ ). In other words, instead of a SPA, we are in a WKB like approximation where one can expand in the slowness of variation of $A(\psi)$, i.e. we can expand in successive derivatives $d^{n} A(\psi) / d \psi^{n}$. This expansion is obtained by successively integrating Eq. (2.39) by parts [using $\left.e^{i \psi} \equiv \frac{d}{d \psi}\left(e^{i \psi} / i\right)\right]$. Contrary to Section II D above we now keep the edge contribution coming from the boundaries. For instance at second order this leads to

$$
I=\left[\frac{e^{i \psi}}{i}\left[A(\psi)+i A^{\prime}(\psi)\right]\right]_{a}^{b}+i^{2} \int_{\psi_{a}}^{\psi_{b}} d \psi A^{\prime \prime}(\psi) e^{i \psi} .
$$

It is easy to re-express this result in terms of the original time variable $t$ by replacing $A(\psi) \equiv a(t) / \dot{\psi}(t)$ and $d / d \psi=$ $(\dot{\psi})^{-1} d / d t$. We deduce from Eq. (3.15) the full 'edge' contribution to the integral $I$ (as explained in Section IID, the 'bulk' contribution is exponentially small):

$$
I_{\text {edge }}=\left[\frac{e^{i \psi}}{i}\left(A(\psi)+i A^{\prime}(\psi)+\cdots+i^{n} A^{(n)}(\psi)+\cdots\right)\right]_{a}^{b} .
$$


This is the explicit form of the parametric expansion sketched in Eq. (2.45). It can be expressed in terms of the time derivatives of $a(t)$ and $\psi(t)$ by using the replacement rules just mentioned. In particular, at the leading and next-to-leading order it reads explicitly

$$
I_{\text {edge }}=\left[\frac{a(t)}{i \dot{\psi}(t)} e^{i \psi(t)}\left\{1+\frac{1}{i \dot{\psi}(t)}\left(\frac{\ddot{\psi}(t)}{\dot{\psi}(t)}-\frac{\dot{a}(t)}{a(t)}\right)\right\}\right]_{t_{a}}^{t_{b}} .
$$

If we apply this general result to $\tilde{h}_{+}(f)$ Eq. (3.12d), we get the estimate

$$
\begin{aligned}
\tilde{h}_{+}(f) & \simeq \tilde{h}_{+}^{\text {edge }}(f) \simeq \frac{a\left(t_{\max }\right)}{i \dot{\psi}_{f}^{+}\left(t_{\max }\right)} e^{i \psi_{f}^{+}\left(t_{\max }\right)}\left[1+\frac{1}{i \dot{\psi}_{f}^{+}\left(t_{\max }\right)}\left(\frac{\ddot{\psi}_{f}^{+}\left(t_{\max }\right)}{\dot{\psi}_{f}^{+}\left(t_{\max }\right)}-\frac{\dot{a}\left(t_{\max }\right)}{a\left(t_{\max }\right)}\right)\right] \\
& \simeq \frac{a\left(t_{\max }\right)}{2 \pi i\left(f+F_{\max }\right)} e^{i \psi_{f}^{+}\left(t_{\max }\right)}\left[1+\frac{1}{2 \pi i\left(f+F_{\max }\right)}\left(\frac{\dot{F}_{\max }}{\left(f+F_{\max }\right)}-\frac{\dot{a}\left(t_{\max }\right)}{a\left(t_{\max }\right)}\right)\right] .
\end{aligned}
$$

Note that, in the approximation where the amplitude is taken as Newtonian-like, i.e., $a(t)=C v_{F}^{2} \propto(F(t))^{2 / 3}$, the last term in Eq. $3.18 \mathrm{~b}$ ) reads $\dot{a}\left(t_{\max }\right) / a\left(t_{\max }\right)=\frac{2}{3} \dot{F}_{\max } / F_{\max }$.

\section{Improved stationary phase approximation when $f<F_{\max }$}

Let us now consider the dominant contribution $\tilde{h}_{-}(f)$, Eq. (3.12b). We start by considering the case where the Fourier variable $f$ [of the Fourier transform of the time-windowed signal Eq. (3.11)] is smaller than $F_{\max }$, but near $F_{\max }$. As will become clear from the formulas below, the interval around $F_{\max }$ where it is needed to improve the usual $\mathrm{SPA}$, is the range,

$$
\left|f-F_{\max }\right| \lesssim(\text { few }) \quad \sqrt{\dot{F}\left(t_{f}\right)} .
$$

In the case where $f$ is in the range Eq. (3.19) with $f<F_{\max }$, there is a saddle-point $t_{f}$ in the first term of the exact integral Eq. (3.3b), and one can still use the parabolic approximation Eq. (3.5a) to the phase $\psi_{f}(t)$, and the lowest approximation Eq. (3.5b) to the amplitude $a(t)$. [Indeed, the work of the previous Section has shown that the local corrections to the integral Eq. 3.4a), coming from the inclusion of more terms in Eqs. (3.5a) and (3.5b), were quite small as long as $N \gtrsim 1$ ]. Therefore, in this case, the resonant contribution to the Fourier transform becomes:

$$
\begin{aligned}
\tilde{h}_{-}(f) & \simeq \int_{-\infty}^{t_{\max }} d t a(t) e^{i \psi_{f}(t)} \\
& \simeq a\left(t_{f}\right) e^{i \psi_{f}\left(t_{f}\right)} \int_{-\infty}^{t_{\max }} d t e^{-i \pi \dot{F}\left(t_{f}\right)\left(t-t_{f}\right)^{2}} .
\end{aligned}
$$

The crucial difference between the Eqs. (3.6) and (3.20b is that the full Gaussian integral has become a complex Fresnel integral which may be evaluated in terms of the complementary error function. Let us recall that the complementary error function $\operatorname{erfc}(z) \equiv 1-\operatorname{erf}(z)$ is defined by

$$
\operatorname{erfc}(z)=\frac{2}{\sqrt{\pi}} \int_{z}^{+\infty} e^{-x^{2}} d x
$$

It takes on the real axis the particular values $\operatorname{erfc}(+\infty)=0$, $\operatorname{erfc}(0)=1$, and $\operatorname{erfc}(-\infty)=2$. By rotating the integration contour in the complex plane $\left(x=-e^{\frac{i \pi}{4}} \xi\right)$ and shifting the new integration variable $\xi$, we get the following useful integration formula:

$$
\int_{-\infty}^{\tau_{m}} d \tau e^{-i\left(a \tau^{2}+2 b \tau+c\right)}=\frac{1}{2} \sqrt{\frac{\pi}{a}} e^{-\frac{i \pi}{4}} e^{i \frac{b^{2}-a c}{a}} \operatorname{erfc}\left[-e^{\frac{i \pi}{4}} \sqrt{a}\left(\tau_{m}+\frac{b}{a}\right)\right] .
$$

The formula Eq. (3.22) motivates us to define the following auxiliary function

$$
\mathcal{C}(\zeta) \equiv \frac{1}{2} \operatorname{erfc}\left(\mathrm{e}^{\mathrm{i} \pi} \frac{1}{4} \zeta\right)
$$


It is useful to note that $\mathcal{C}(+\infty)=0, \mathcal{C}(0)=1 / 2$, and $\mathcal{C}(-\infty)=1$. Moreover, the leading terms in the asymptotic expansions of $\mathcal{C}(\zeta)$ as $\zeta \rightarrow \pm \infty$ are

$$
\begin{aligned}
& \zeta \rightarrow-\infty, \quad \mathcal{C}(\zeta) \sim 1+\frac{e^{-\frac{i \pi}{4}}}{2 \sqrt{\pi}} \frac{e^{-i \zeta^{2}}}{\zeta}, \\
& \zeta \rightarrow+\infty, \quad \mathcal{C}(\zeta) \sim \frac{e^{-\frac{i \pi}{4}}}{2 \sqrt{\pi}} \frac{e^{-i \zeta^{2}}}{\zeta} .
\end{aligned}
$$

As the auxiliary function $\mathcal{C}(\zeta)$ plays an important role in our work we plot it in Fig. 5 . From the Figure we note that the real part of the above complex function is a softened version of a step function $\theta(-\zeta)$ and the imaginary part an oscillating function vanishing in the limits $\pm \infty$, as well as at $\zeta=0$.

Armed with this definition one finds that the right hand side of Eq. (3.20b) yields the approximation

$$
\begin{aligned}
\tilde{h}_{-}(f) & \simeq \mathcal{C}\left(\zeta_{0}(f)\right) \tilde{h}^{\mathrm{uspa}}(f), \\
\text { where, } \zeta_{0}(f) & \equiv \sqrt{\pi \dot{F}\left(t_{f}\right)}\left(t_{f}-t_{\max }\right) .
\end{aligned}
$$

In words, when $f<F_{\max }$ one can correct for the 'edge effects' caused by the cutoff at $t_{\max }$ by multiplying the usual SPA $\tilde{h}^{\text {uspa }}(f)$ given in Eq. (3.7) by a complex 'correction factor' $\mathcal{C}\left(\zeta_{0}(f)\right)$. The expression Eq. (3.25a) gives very good overlaps with the exact discrete Fourier transform (DFT) of the time-windowed signal Eq. (3.11). However, it is possible to do even better by a slight modification of the argument $\zeta_{0}(f)$, Eq. (3.25b).

To understand how a slight modification of the argument $\zeta(f)$ of the auxiliary function $\mathcal{C}(\zeta)$ can improve both the visual agreement (even quite far away from $F_{\max }$ ) and the overlap with the exact DFT of the time-windowed signal Eq. (3.11) we have to take into account the asymptotic expansion Eq. (3.24a). Indeed, on the one hand, when inserting the expansion Eq. (3.24a) into Eq. (3.25a), using Eq. (3.7) for $\tilde{h}^{\text {uspa }}(f)$ and allowing for a more general frequency dependent argument $\zeta(f)$, we find that $\tilde{h}(f)$ differs from $h^{\text {uspa }}(f)$ (in the domain $\zeta(f) \rightarrow-\infty$ i.e. $f \ll F_{\max }$ ) by a correction term proportional to $e^{-i \pi / 2} e^{i\left[\psi_{f}\left(t_{f}\right)-\zeta^{2}\right]} / \zeta$. On the other hand, a different way of estimating this edge correction consists in writing Eq. (3.20b) as an integral between $-\infty$ and $+\infty$ minus a "correcting" integral between $t_{\max }$ and $+\infty$. As was discussed in Section [IID the latter integral can be estimated by successive integration by parts. This gives [see Eq. (3.17)] a first order correction term proportional to $e^{+i \pi / 2} a\left(t_{\max }\right) e^{i \psi_{f}\left(t_{\max }\right)} / \dot{\psi}_{f}\left(t_{\max }\right)$. The phasing of this edge correction can be made to agree perfectly with the phasing predicted by the form Eq. (3.25a) written with a generalized argument $\zeta(f)$ if $\left(\psi_{f}\left(t_{f}\right)-\zeta^{2}\right)=\psi_{f}\left(t_{\max }\right)$. This leads us to define, in the domain $\zeta<0$, i.e. $f<F_{\max }$, the new argument

$$
\zeta_{<} \equiv-\sqrt{\psi_{f}\left(t_{f}\right)-\psi_{f}\left(t_{\max }\right)} .
$$

In the left part of the crucial region Eq. (3.19) the argument $\zeta_{<}(f)$ Eq. (3.26) is nearly identical to the previous result $\zeta_{0}(f)$ Eq. (3.25b), as is seen from Eq. (3.5a). However, we have checked that the replacement of $\zeta_{0}$ by $\zeta_{<}$improves both the visual agreement and the overlap with the exact $\tilde{h}(f)$. Let us also note in passing that an amplitude proportional to $\zeta^{-1}$ of the correction term to $\tilde{h}^{\text {uspa }}(f)$ derived from the asymptotic expansion of Eq. (3.24a) is consistent with the different analytical treatment used in [31] which was valid only for $f \ll F_{\max }$, i.e. large, negative $\zeta$. By contrast our approach based on the function $\mathcal{C}(\zeta)$ is adequate in the full range $-\infty<\zeta \leq 0$ without exhibiting any fictitious blowup at $\zeta=0$ (remember $\mathcal{C}(0)=1 / 2)$. [Our approach is also valid in the region $\zeta>0$, i.e. $f>F_{\max }$, but for an improved treatment of this domain, we shall find it convenient to modify further the argument $\zeta(f)$ in the following Section.]

Summarizing: we propose as final result for the resonant part of our improved SPA for Newtonian-like signals (or inSPA)

$$
f \leq F_{\max }: \quad \tilde{h}_{-<}^{\text {inspa }}=\mathcal{C}\left(\zeta_{<}(f)\right) \frac{a\left(t_{f}\right)}{\sqrt{\dot{F}\left(t_{f}\right)}} e^{i\left(\psi_{f}\left(t_{f}\right)-\pi / 4\right)} .
$$

The corresponding total improved approximation $\tilde{h}^{\text {intot }}$ to the Fourier transform $\tilde{h}=\tilde{h}_{-}(f)+\tilde{h}_{+}(f)$ is the sum of Eqs. (3.18b) and (3.27). Note that the ratio $\tilde{h}_{-} / \tilde{h}_{+}$is (when $t_{f}$ is near $t_{\max }$ ) of order $4 \pi F_{\max } / \sqrt{\dot{F}_{\max }}=4 \pi \sqrt{N_{\max }}$ (this is consistent with the $\varepsilon$ scaling of Eqs. (2.44) and (2.45), remembering that $\varepsilon \sim 1 / 2 \pi N)$. The contribution of $\tilde{h}_{+}$is expected to be non-negligible only for signals which are really discontinuous in time. As the real signal (whatever be the subsequent plunge signal) will be continuous (and even smooth) it is clear that one should not add any contribution from $\tilde{h}_{+}$when applying our above treatment to real signals. In fact, we shall see below that, even for discontinuous signals, the addition of $h_{+}$has only a minute effect on overlaps. 


\section{E. Approximate Fourier transform when $f>F_{\max }$}

Let us now consider the evaluation of $\tilde{h}_{-}(f)$ in the case when the Fourier variable $f$ is larger than $F_{\max }$ [but near $F_{\max }$, in the sense of Eq. (3.19)]. In that case the integral Eq. (3.12b) giving $\tilde{h}_{-}(f)$ no longer has a saddle-point. However, it 'nearly' has a saddle-point and therefore we expect that

$$
\tilde{h}_{-}(f)=\int_{-\infty}^{t_{\max }} d t a(t) e^{i \psi_{f}(t)}
$$

will still dominate over $\tilde{h}_{+}(f)$. One could think of two ways of analytically approximating the integral Eq. (3.28). A first way is to still use the fact that (for Newtonian-like signals where the mathematical function $F(t)$ continues to exist and increase beyond $t=t_{\max }$ ) though there is no saddle-point in the domain of integration $\left[-\infty, t_{\max }\right]$, there exists a nearby saddle-point of the analytically continued phase function $\psi_{f}(t)$. More precisely, for Newtonian-like signals the mathematical equation $F\left(t_{f}\right)=f$ still defines a unique value $t_{f}$ (with $t_{f}>t_{\max }$ when $f>F_{\max }$ ). Capitalizing on the existence of this nearby saddle-point one can still try to insert the expansions Eqs. (3.5a) and (3.5b). This leads to a result of the form Eq. (3.27) with the correction factor Eq. (3.25b), i.e. now considered for positive values of the argument $\left.\zeta_{0}(f) \equiv \sqrt{\pi \dot{F}\left(t_{f}\right)\left(t_{f}\right.}-t_{\text {max }}\right)$. In other words, a simple uniform expansion to $\tilde{h}_{-}(f)$ on both sides of $f \sim F_{\max }$ would seem to be simply

$$
\begin{aligned}
\tilde{h}_{-}^{\text {cspa }}(f) & =\mathcal{C}\left(\zeta_{0}(f)\right) \frac{a\left(t_{f}\right)}{\sqrt{\dot{F}\left(t_{f}\right)}} e^{i\left[\psi_{f}\left(t_{f}\right)-\pi / 4\right]}, \\
\text { with } \zeta_{0}(f) & =\sqrt{\pi \dot{F}\left(t_{f}\right)}\left(t_{f}-t_{\max }\right) .
\end{aligned}
$$

Here 'cspa' means (zeroth order) corrected SPA. Note that when $f>F_{\max }, t_{f}$, and therefore all the quantities evaluated at $t_{f}$, are defined by using the (supposedly existing) analytic continuation of the mathematical function $F(t)$ beyond $t=t_{\max }$.

In our first attempts at improving the SPA in presense of a time-windowing we came up with the simple proposal Eqs. (3.29a)-(3.29b) and it gave excellent overlaps with the exact Fourier transform. However, we realised later that we could further improve on this simple proposal. We already stated that for $f<F_{\max }$ our best proposal is to modify the argument Eq. (3.29b) into Eq. (3.26). In the case where $f>F_{\max }$ our best proposal is neither to use the straightforward argument Eq. (3.29b), nor the "improved-phasing" argument Eq. (3.26) with a positive sign in front of the square-root [which, however, still improves over the choice Eq. (3.29b)] but to follow a different tack which will turn out to be useful when considering the case of relativistic-like signals in the next Section.

To motivate our proposal in the case $f>F_{\max }$, let us remark that the integral to be approximated, i.e. Eq. (3.28) having no saddle-point in the domain of integration, is formally of the general type Eq. (3.14) with the phase $\psi(t)=\psi_{f}(t)$ being a monotonically increasing function of $t$. The important information we wish to deduce from Eqs. (3.15) and (3.17) is that there exists an expansion (valid when $f \gg F_{\max }$, i.e. $\zeta_{0}(f)$ is large and positive) in which $\tilde{h}_{-}(f)$ is entirely expressed in terms of the values of the functions $\psi_{f}(t)$ and $a(t)$, and their derivatives, evaluated at the edge point $t=t_{\max }$. This contrasts with the 'corrected' result Eqs. (3.29a)-(3.29b) which relied on the existence of the functions $\psi_{f}(t)$ and $a(t)$ in the "unphysical" region $t>t_{\max }$. This motivates us to look for an approximation to Eq. (2.39) valid all over the domain $\zeta_{0}(f)>0$ [and not only when $\zeta_{0} \gg 1$ which will be seen to be the domain of validity of Eqs. (3.15) and (3.17)] but expressed entirely in terms of the edge values of $\psi_{f}(t)$ and $a(t)$. We propose to define such an approximation by replacing the phase and amplitude in Eq. (3.28) by

$$
\begin{aligned}
\psi_{f}(t) & \simeq \psi_{f}\left(t_{\max }\right)+2 \pi\left(f-F_{\max }\right)\left(t-t_{\max }\right)-\pi \dot{F}\left(t_{\max }\right)\left(t-t_{\max }\right)^{2}, \\
a(t) & \simeq a\left(t_{\max }\right) .
\end{aligned}
$$

Thanks to the parabolic nature of the approximation Eq. (3.30a this again leads to an incomplete complex Gaussian integral (i.e. a Fresnel integral) which can be evaluated as before in terms of the complementary error function. Using Eq. (3.22), this leads to our final proposal for the (nearly) resonant part of $\tilde{h}_{-}(f)$ for Newtonian-like signals

$$
\begin{aligned}
f \geq F_{\max }: \tilde{h}_{->}^{\text {inspa }}(f) & =\mathcal{C}\left(\zeta_{>}(f)\right) \frac{a\left(t_{\max }\right)}{\sqrt{\dot{F}\left(t_{\max }\right)}} \exp i\left[\psi_{f}\left(t_{\max }\right)+\frac{\pi\left(f-F_{\max }\right)^{2}}{\dot{F}\left(t_{\max }\right)}-\pi / 4\right], \\
\zeta_{>}(f) & =\frac{\sqrt{\pi}\left(f-F_{\max }\right)}{\sqrt{\dot{F}\left(t_{\max }\right)}} .
\end{aligned}
$$


Note that, in the parabolic approximation where Eq. (3.5a) or Eq. (3.30a hold, the function $\zeta_{>}(f)$ is approximately equal both to $\zeta_{0}(f)$, Eq. (3.29b), and to the analytic continuation of Eq. (3.26), i.e. $\zeta_{<}(f) \equiv$ $\operatorname{sign}\left(f-F_{\max }\right) \sqrt{\psi_{f}\left(t_{f}\right)-\psi_{f}\left(t_{\max }\right)}$. Note also that the phase factor in Eq. (3.31a) (which is explicitly expressed in terms of edge quantities) is nearly equal to the analytic continuation of the usual SPA phase factor appearing in Eq. (3.27), i.e. $\exp \left[i \psi_{f}\left(t_{f}\right)-i \pi / 4\right]$. Finally, as required, the expressions, Eqs. (3.27) and (3.31a) match continuously at $f=F_{\max }$ with common value

$$
\tilde{h}_{-<}^{\text {inspa }}\left(F_{\max }\right)=\tilde{h}_{->}^{\text {inspa }}\left(F_{\max }\right)=\frac{1}{2} \frac{a\left(t_{\max }\right)}{\sqrt{\dot{F}\left(t_{\max }\right)}} e^{i\left(\psi_{f}\left(t_{\max }\right)-\pi / 4\right)} .
$$

Summarizing: our best analytical estimate for the Fourier transform of discontinuous Newtonian-like signals is the sum

$$
\tilde{h}^{\text {intot }}(f)=\tilde{h}_{-}^{\text {inspa }}(f)+\tilde{h}_{+}^{\text {edge }}(f),
$$

where $\tilde{h}_{+}^{\text {edge }}(f)$ is approximated by Eq. (3.18b) and where $\tilde{h}_{-}^{\text {inspa }}(f)$ is given, when $f \leq F_{\max }$ by Eq. (3.27) and for $f \geq F_{\max }$ by Eq. (3.31a). As stated earlier, we shall in fact recommend that the edge correction $h_{+}^{\text {edge }}$ be not included when applying our result to real signals (we shall also see that it brings only a negligible improvement to the overlaps of time-windowed signals).

\section{F. Comparison between the improved SPA, the usual SPA and the 'exact' SPA (numerical DFT)}

Before proceeding to a quantitative comparison of the various approximants in Table III, it is important to remark that one needs to be more specific when using the terminology uSPA. One could compute the uSPA truncated at the $F_{\mathrm{LSO}}$ that we refer to as uSPAw ( where 'w' stands for 'windowed') or the uSPA truncated at the Nyquist frequency that we designate as uSPAn (where 'n' stands for 'Nyquist'). In Table [I] we have listed the overlaps, as defined by Eq. (2.8), of a signal model generated in the time-domain and then Fourier transformed using a numerical DFT algorithm with the same signal model but directly generated in the frequency-domain using the usual SPA (uSPA), the corrected SPA (cSPA) and the improved Newtonian SPA (inSPA) discussed earlier. For simplicity, we consider only equal-mass systems $(\eta=1 / 4)$ and parametrize them by the total mass $m=m_{1}+m_{2}$. The total mass is the crucial parameter which measures the location of the $F_{\mathrm{LSO}}$ with respect to the bandwidth of the detector. The parameter $\eta$ is also important because it determines the number of cycles near the LSO [Eq. (2.27) shows that $N\left(F_{\mathrm{LSO}}\right)$ scales as $\left.1 / \eta\right]$. The worst case (for the sensibility to the shutting off of the signal after the LSO) is $\eta=\eta_{\max }=1 / 4$, and this is why we focus on this case. [We are also motivated by the fact that $1 / 4$ being the maximum value of the function $\eta\left(m_{1}, m_{2}\right)$, the observed values of $\eta$, corresponding to a random sample of of $m_{1}$ and $m_{2}$, are expected to have an accumulation point at $\eta_{\max }=1 / 4$.] As we have checked, if our filters exhibit good overlaps for $\eta=1 / 4$ they will have even better overlaps for $\eta<1 / 4$ and the same value for $m$.

The error function needed in computing $\mathcal{C}(f)$ is numerically computed using the NAG library S15DDF. The overlaps are shown for the usual SPA with a frequency-windowing (uSPAw) together with the overlaps for the uSPAn, cSPA and inSPA, computed up to $F_{\text {Nyquist }}$. Table II shows that the improvements on the SPA that we propose in this paper (both the simple cspa and our final inspa) succeed very well in modelling the edge effects due to time-windowing. The overlaps in the case of cSPA/inSPA are better than 0.99 for equal-mass systems with total mass $m<40 M_{\odot}$. For a system of $m=40 M_{\odot}$ uSPAw gives an overlap of 0.8589 resulting in a loss in the number of events by $37 \%$. Although the overlaps of cSPA and inSPA seem to be always about the same, we think that inSPA is a better representation of $\tilde{h}(f)$; it has better overlaps in the case of the most massive systems (see the first lines of Table II]), and, as shown by Fig. 6, it captures better the decay of $\tilde{h}(f)$ beyond $F_{\max }$. In the Table for the usual SPA we have listed the overlaps for uSPAw i.e., uSPA terminated at $f=F_{\mathrm{LSO}}=4400\left(m_{\odot}\right)^{-1} \mathrm{~Hz}$ and the overlap (uSPAn) up to the Nyquist frequency $f_{\text {Nyquist }}=2 \mathrm{kHz}$. As is very clear from these entries, windowing of the SPA improves the overlaps for massive systems very much. As remarked earlier, this is why in DIS, while comparing the DFT to the SPA, the uSPAw was used. On the other hand, computing overlaps up to the Nyquist frequency, i.e. uSPAn, produces much smaller overlaps.

\footnotetext{
${ }^{6}$ This defines for us the 'exact' Fourier representation of the signal, after due care has been taken to use a smooth time-window below $f_{s}$, and a high enough sampling rate.
} 
To understand this further we plot in Fig. 6 the power per logarithmic bin of the squared SNR, $d \rho^{2} / d \log f=$ $f|\tilde{h}(f)|^{2} / S_{n}(f)$, which is the Fourier-domain quantity of most significance when discussing overlaps. We compare this quantity for various approximations to the Fourier-transform of an (arbitrarily-normalized) time-windowed signal: DFT, uSPA, cSPA and inSPA. In the important range of frequencies our best analytical approximant inSPA agrees with the exact result (FFT) quite well, the uSPA grossly overestimates and cSPA somewhat underestimates the actual signal power. This is why, though analytically continued up to $f_{\text {Nyquist }}$, the uSPAn returns a smaller overlap as compared to the windowed SPA (uSPAw) because it overestimates the power in the signal beyond $F_{\text {LSO }} 16$.

In all the comparisons above, it is worth stressing that the FFT calculation is delicate: The 'exact' time-domain chirp contains an infinite number of cycles in the far past, with instantaneous frequencies tending to zero. As what happens to frequencies below the seismic cut-off $f_{\mathrm{s}}=40 \mathrm{~Hz}$ is not physically important, we wish to simplify the numerical calculation of the FFT by essentially discarding the (infinite) part of the signal, having instantaneous frequencies $F(t)<f_{\mathrm{s}} \equiv F\left(t_{\mathrm{s}}\right)$. We started doing that by simply time-windowing the signal for $t<t_{\min }<t_{\mathrm{s}}$ by a sharp, lower time-window $\theta\left(t-t_{\min }\right)$. However, this method introduces physically spurious oscillations (which are the lower-cutoff analogue of the physically important upper-cutoff oscillations) present in both $\tilde{h}_{+}(f)$ and $\tilde{h}_{-}(f)$. One way to deal with this problem is to subtract out from the FFT these spurious edge oscillations by using the general formula Eq. (3.17), which in the present context, can be applied both to $\tilde{h}_{+}^{\mathrm{FFT}}(f)$ and $\tilde{h}_{-}^{\mathrm{FFT}}(f)$. For instance, to lowest order the FFT corrected for these oscillations would read

$$
\begin{aligned}
\tilde{h}_{\mathrm{corrected}}^{\mathrm{FFT}} & =\tilde{h}^{\mathrm{FFT}}+\Delta_{\text {min }}^{+}+\Delta_{\text {min }}^{-}, \\
\text {where, } \Delta_{\min }^{ \pm} & =\frac{a\left(t_{\min }\right)}{2 \pi i\left(f \pm F_{\min }\right)} e^{i \psi_{f}^{ \pm}\left(t_{\min }\right)},
\end{aligned}
$$

where $F_{\min }=F\left(t_{\min }\right)<f_{\mathrm{s}}$ [a better approximation can be derived from Eq. (3.17)]. However, it seems better to use an alternative approach which does not require one to correct by hand the FFT. The alternative approach we have actually used in our calculations consists in imposing a smooth (rather than a sharp) lower time-window on the exact chirp, acting below $t_{\mathrm{s}}$ and in a smooth-enough manner that it does not introduce spurious edge-oscillations in the frequency-domain. The smooth time-window that we used consists in multiplying the chirp by the function

$$
\sigma\left(t, t_{1}, t_{2}\right)=\frac{1}{e^{z}+1}, \quad z=\frac{t_{2}-t_{1}}{t-t_{1}}+\frac{t_{2}-t_{1}}{t-t_{2}}
$$

which smoothly interpolates between 0 when $t=t_{1}+0$ and 1 when $t=t_{2}-0$. We used $t_{1}$ such that $F_{1}=F\left(t_{1}\right)=30$ $\mathrm{Hz}$ and $t_{2}=t_{\mathrm{s}}$ i.e. $F\left(t_{2}\right)=f_{\mathrm{s}}$. Moreover, we need to be careful with sampling and phase factors to correctly reproduce the edge correction to $\tilde{h}_{+}^{\text {edge }}$.

In addition to the comparative evaluation of the various approximants, Table II also provides a numerical proof regarding the effect of $h_{+}^{\text {edge }}$ on the overlaps. It is quite important to note that the inclusion of the non-resonant edge term $h_{+}^{\text {edge }}$ has only a very minute (but positive) effect on overlaps. This is good news for our formal time-windowing ansatz, because we expect that this contribution will be (exponentially) negligible in the case of real (continuous) signals. We interpret the fact that even for our formal discontinuous model $h_{+}^{\text {edge }}$ is negligible 7 as a confirmation that our improved SPA can adequately model not only signals that vanish after the LSO, but also signals that shut off rather quickly (on the $F_{\text {LSO }}^{-1}$ time-scale) after the LSO. It leads us also to propose, finally, to use as analytical representative of the FT of real signals the $h_{-}^{\text {inspa }}$ part of our formula above (without the edge term). [To simplify the notation, we shall henceforth drop the extra subscript minus on $h^{\text {inspa }}$.]

In computing the above overlaps we have matched all the parameters of the two waveforms, including the time of arrival and the starting phase . The overlaps in this Table as well as all other Tables in this paper are found to be insensitive to the sampling rate at the level of a fraction of a percent, provided that it is large enough to obey Shannon's sampling theorem.

\footnotetext{
${ }^{7}$ Note that our statement here is only that $h_{+}^{\text {edge }}(f)$ can be effectively omitted without significantly worsening the overlaps. We are not claiming that $h_{+}^{\text {edge }}(f)$ is pointwise numerically negligible compared to $h_{-}(f)$. Indeed, because the instantaneous number of cycles is rather small near the LSO, our analytical estimates above show that $h_{+}^{\text {edge }}(f)$ is not very much smaller than $h_{-}(f)$ near $f=F_{\text {LSO }}$.

${ }^{8}$ The lag is set equal to zero in testing the accuracy of the Fourier representation but chosen optimally when testing faithfulness of a family of templates e.g. in Sec. $\mathrm{V}$.
} 


\section{IMPROVED STATIONARY PHASE APPROXIMATION FOR RELATIVISTIC SIGNALS IN THE ADIABATIC APPROXIMATION: THE SPP-APPROXIMANTS}

Though one might a priori think that it is a simple matter to generalize the improved SPA discussed above for Newtonian-like signals to the relativistic case, it does not turn out to be so. What complicates matters is that there are serious qualitative, "non-perturbative" differences between the two cases: first, the value of $\dot{F}(t)$ formally tends to $+\infty$ at the last stable orbit (LSO) which physically defines the upper-cutoff $t_{\max }$ of the inspiral signal, and, second, the mathematical function $F(t)$ does not admit a unique real analytic continuation beyond $t_{\text {max }}=t_{\text {LSO }}$. (These two facts are evidently related; indeed we shall see that $F(t)$ behaves in the non-analytic manner $F(t) \sim c_{1}+c_{2}\left(t_{\mathrm{LSO}}-t\right)^{\frac{1}{2}}$ when $t \rightarrow t_{\text {LSO }}^{-}$). Remembering the crucial role of a finite $\dot{F}(t)$ in the results Eqs. (3.27) and (3.31a), it is clear that we need to tackle afresh the problem of finding a good, analytic approximation to $\tilde{h}_{-}(f)$. Similarly, in view of the appearance of $\dot{F}^{+1}\left(t_{\max }\right)$ in the next-to-leading contribution to $\tilde{h}_{+}(f)$ Eq. $(3.18 \mathrm{~b})$, we shall also need to revisit the calculation of $h_{+}(f)$ (though we shall, again, find that it makes only a negligible contribution to the overlaps.)

\section{A. The phasing formula for relativistic signals in the adiabatic approximation}

To extend the treatment of the previous Section and go beyond the Newtonian approximation, let us begin with the phasing formulas for gravitational waves from compact binaries written in a parametric form in terms of the variable $v_{F} \equiv(\pi m F)^{1 / 3}$ defined by the total mass $m=m_{1}+m_{2}$ and instantaneous gravitational wave frequency $F$

$$
\begin{gathered}
t\left(v_{F}\right)=t_{\mathrm{LSO}}+m \int_{v_{F}}^{v_{\mathrm{LSO}}} d v \frac{E^{\prime}(v)}{\mathcal{F}(v)}, \\
\phi\left(v_{F}\right)=\phi_{\mathrm{LSO}}+2 \int_{v_{F}}^{v_{\mathrm{LSO}}} d v v^{3} \frac{E^{\prime}(v)}{\mathcal{F}(v)},
\end{gathered}
$$

where $E(v)$ is the dimensionless energy function related to the total relativistic energy or Bondi mass by $E_{\text {total }}=$ $m(1+E), \mathcal{F}(v)$ the flux function denoting the gravitational wave luminosity of the system and $t_{\mathrm{LSO}}$ is the time and $\phi_{\mathrm{LSO}}$ is the phase of the signal when $v=v_{\mathrm{LSO}}$. The parametric representaion Eqs. (4.1 4.2 of the phasing formula $\phi=\phi(t)$ holds under the assumption of 'adiabatic inspiral', i.e., that gravitational radiation damping can be treated as an adiabatic perturbation of a circular motion. See 43. for a treatment of radiation damping going beyond this approximation.

In the restricted post-Newtonian approximation, one uses a Newtonian approximation for the amplitude [25]. However, in order to extract an inspiral signal that may be buried in noisy data by the method of matched filtering, we need to employ post-Newtonian accurate representations for the two functions $E^{\prime}(v)$ and $\mathcal{F}(v)$ that appear in the above phasing formulas. To any approximant $E_{A}(v), \mathcal{F}_{A}(v)$, correspond [by replacing $E(v) \rightarrow E_{A}(v), \mathcal{F}(v) \rightarrow \mathcal{F}_{A}(v)$ in Eqs. (4.1) and (4.2)] some approximate parametric representation $t=t_{A}\left(v_{F}\right), \phi=\phi_{A}\left(v_{F}\right)$, and therefore a corresponding approximate time-domain template

$$
h^{A}=h^{A}\left(t ; \mathcal{C}, t_{\mathrm{LSO}}, \phi_{\mathrm{LSO}}, m, \eta\right)
$$

obtained by replacing $v_{F}$, in the following $v_{F}$-parametric representation of the waveform

$$
h^{A}\left(v_{F}\right)=\mathcal{C} v_{F}^{2} \cos \phi_{A}\left(v_{F}\right),
$$

by the function of time $v_{F}=v_{A}(t)$ obtained by inverting $t=t_{A}\left(v_{F}\right)$.

The standard approximants for $E(v)$ and $\mathcal{F}(v)$ are simply their successive Taylor approximants $E_{T_{n}}$ and $\mathcal{F}_{T_{n}}$ respectively. The DIS strategy for constructing new approximants to $E(v)$ and $\mathcal{F}(v)$ is two-pronged: Starting from the more basic energy-type and flux-type functions, $e(v)$ and $l(v)$ [13] we construct Padé-type approximants, say $e_{P_{n}}, l_{P_{n}}$, of the "basic" functions $e(v), l(v){ }^{9}$. We then compute the required energy and flux functions entering the phasing formula. The successive approximants $E\left[e_{P_{n}}\right]$ and $\mathcal{F}\left[e_{P_{n}}, l_{P_{n}}\right]$ have better convergence properties than their

\footnotetext{
${ }^{9}$ For explicit formulas representing $E(v)$ and $\mathcal{F}(v)$ see Eqs. (3.8),(4.2) and (4.3) of DIS. The associated $e(v)$ and $l(v)$ functions are given by Eqs. (3.7), (3.9) and Eqs. (4.4)-(4.9) in DIS . See also Eqs. (3.5), (3.11) and Eqs. (3.18)-(3.23) there.
} 
Taylor counterparts $E_{T_{n}}\left[e_{T_{n}}\right]$ and $\mathcal{F}_{T_{n}}\left[e_{T_{n}}, l_{T_{n}}\right]$. In DIS we were working directly with the time-domain signal $h(t)$. As explained above this necessarily requires a numerical inversion of the parametric representation $t=t\left(v_{F}\right)$. By contrast, if one wants to compute the usual stationary phase approximation of $h(t)=\mathcal{C} v_{F}^{2}(t) \cos \phi\left(v_{F}(t)\right)$ there is no need to invert this parametric representation. Indeed, from Eq. (3.7), it is sufficient to know the instantaneous amplitude and the phase at the time $t_{f}$ where $f=F\left(t_{f}\right)$. This time is simply given by the same expression Eq. (4.1) as above with the replacement of $v_{F} \equiv(\pi m F)^{1 / 3}$ by $v_{f} \equiv(\pi m f)^{1 / 3}$, i.e. the stationary point $t_{f}$ is given by

$$
t_{f}=t_{\mathrm{LSO}}+m \int_{v_{f}}^{v_{\mathrm{LSO}}} \frac{E^{\prime}(v)}{\mathcal{F}(v)} d v .
$$

One then substitutes this value of $t_{f}$ in Eq. (4.2) to compute the phase $\psi_{f}\left(t_{f}\right) \equiv 2 \pi f t_{f}-\phi\left(t_{f}\right)$ of the Fourier component:

$$
\psi_{f}\left(t_{f}\right)=2 \pi f t_{\mathrm{LSO}}-\phi_{\mathrm{LSO}}+2 \int_{v_{f}}^{v_{\mathrm{LSO}}}\left(v_{f}^{3}-v^{3}\right) \frac{E^{\prime}(v)}{\mathcal{F}(v)} d v .
$$

In terms of these quantities one has

$$
\tilde{h}^{\text {uspa }}(f)=\frac{1}{2} \mathcal{C} \frac{v_{f}^{2}}{\sqrt{\dot{F}\left(t_{f}\right)}} e^{i\left[\psi_{f}\left(t_{f}\right)-\frac{\pi}{4}\right]}
$$

The inclusion of relativistic effects in $\tilde{h}^{\text {uspa }}(f)$ is then simply accomplished by using relativistic accurate expressions for $E^{\prime}(v)$ and $\mathcal{F}(v)$ in the formulas giving $\psi_{f}\left(t_{f}\right)$ and $\dot{F}\left(t_{f}\right)$. The coefficient $\mathcal{C}$, in Eq. (4.4), determining the actual amplitude of the waveform reads:

$$
\mathcal{C}(r, i, \theta, \bar{\phi}, \bar{\psi})=(4 \eta)\left(\frac{m}{d}\right) C(i, \theta, \bar{\phi}, \bar{\psi}),
$$

where $d$ is the distance to the source, and where

$$
\begin{aligned}
C(i, \theta, \bar{\phi}, \bar{\psi}) & =\sqrt{A^{2}+B^{2}} \\
\text { with, } A & =\frac{1}{2}\left(1+\cos ^{2} i\right) F_{+} ; B=\cos i F_{\times},
\end{aligned}
$$

with the beam-pattern factors

$$
\begin{aligned}
F_{+}(\theta, \bar{\phi}, \bar{\psi}) & =\frac{1}{2}\left(1+\cos ^{2} \theta\right) \cos 2 \bar{\phi} \cos 2 \bar{\psi}-\cos \theta \sin 2 \bar{\phi} \sin 2 \bar{\psi} \\
F_{\times}(\theta, \bar{\phi}, \bar{\psi}) & =\frac{1}{2}\left(1+\cos ^{2} \theta\right) \cos 2 \bar{\phi} \sin 2 \bar{\psi}+\cos \theta \sin 2 \bar{\phi} \cos 2 \bar{\psi}
\end{aligned}
$$

In these formulas the angle $i$ denotes the inclination of the orbit with respect to the plane of the sky, and the angles $\theta, \bar{\phi}$, and $\bar{\psi}$ parametrize both the propagation direction and the polarization of the gravitational wave with respect to the detector (see [5] for exact definitions; we added a bar over $\phi$ and $\psi$ to distinguish them from the GW phase $\phi$ and Fourier phase $\psi$ respectively). Performing averages over the angles in the squared SNR leads to :

$$
\left\langle F_{+}^{2}\right\rangle_{\theta, \bar{\phi}, \bar{\psi}}=\left\langle F_{\times}^{2}\right\rangle_{\theta, \bar{\phi}, \bar{\psi}}=\frac{1}{5},
$$

and finally

$$
\left\langle C^{2}\right\rangle_{i, \theta, \bar{\phi}, \bar{\psi}}=\frac{4}{25}
$$

We are finally in a position to write down the rms and ideal SNRs. For a binary at a distance $d$ from the earth consisting of stars of individual masses $m_{1}$ and $m_{2}$ (total mass $m \equiv m_{1}+m_{2}$ and symmetric mass ratio $\eta=m_{1} m_{2} / m^{2}$ ) the rms and ideal SNRs, obtained by using the rms and ideal values of $C$, namely $C=2 / 5$ and $C=1$, respectively, when replacing Eq. (4.7) in Eq. (2.20), or equivalently, when replacing $a(f)=(1 / 2) \mathcal{C} v^{2}(f)=2 \eta m d^{-1} C v^{2}(f)$ in Eq. (2.22) (with Eq. (2.26) and a truncation at $F_{\mathrm{LSO}}$ ), are given by 


$$
\rho_{\mathrm{rms}}=\frac{m^{5 / 6}}{d \pi^{2 / 3}}\left(\frac{\eta}{15}\right)^{1 / 2}\left[\int_{0}^{F_{\mathrm{LSO}}} d f \frac{f^{-7 / 3}}{S_{n}(f)}\right]^{1 / 2}, \quad \rho_{\text {ideal }}=\frac{5}{2} \rho_{\mathrm{rms}} .
$$

Note that the SNR depends only on the combination $\mathcal{M}=m \eta^{3 / 5}$ - the chirp mass (see e.g. [19]), and that the first Eq. (4.13) is equivalent to Eq. (1.8).

Let us next delineate the qualitative differences between the relativistic and non-relativistic cases by considering the function appearing as denominator in the uSPA, Eq. (4.7)

$$
\dot{F}(t)=\frac{1}{2 \pi} \frac{d^{2} \phi}{d t^{2}}=-\frac{3 v^{2}}{\pi m^{2}} \frac{\mathcal{F}(v)}{E^{\prime}(v)} .
$$

At the LSO, the gravitational wave flux $\mathcal{F}(v)$ is finite (it blows up only later, when reaching the light ring 13 ) while, by definition, $E^{\prime}(v)$ vanishes linearly, $E^{\prime}(v) \propto v-v_{\text {LSO }}$. As we shall see below this means that $\dot{F}(t)$ blows up as $\left(t_{\mathrm{LSO}}-t\right)^{-1 / 2}$. A consequence of this blow up is that the last two terms in Eq. (3.9p) blow up like $\left(t_{\mathrm{LSO}}-t\right)^{-3 / 2}$ confirming the need for a special treatment of the Fourier transform near the LSO. We are here speaking of the exact behaviour of the functions $E(v)$ and $\mathcal{F}(v)$, as supposedly known from combining the test-mass limit results [39] with the best available results on the physics underlying the existence of the LSO [13], and the emission of gravitational waves in comparable mass systems [24]. In DIS, we have incorporated this information so that all the P-approximants $E_{P_{n}} \equiv E\left(e_{P_{n}}\right), \mathcal{F}_{P_{n}} \equiv \mathcal{F}\left[e_{P_{n}}, l_{P_{n}}\right]$ that we define share, with the "exact" functions $E$ and $\mathcal{F}$ the crucial properties mentioned above (i.e. finite $\mathcal{F}\left(v_{\mathrm{LSO}}\right)$ and $\left.E^{\prime}(v) \propto v-v_{\mathrm{LSO}}\right)$. The (less-convergent) successive T-approximants $E_{T_{n}}$ and $\mathcal{F}_{T_{n}}$ do not incorporate this information exactly, and only as $n$ increases they tend to incorporate it. In our opinion the $T_{n}$ approximants disqualify as 'relativistic' approximants since they do not consistently incorporate the expectation (based on several different methods; see references in 40]) that the frequency at the LSO is (for any $\eta \leq 1 / 4)$ numerically near the Schwarzschild-like prediction, Eq. (2.14). Indeed, if we define the 2PN Taylor estimate of $F_{\mathrm{LSO}}$ by the value of $v=(\pi m F)^{1 / 3}$ where the straightforward Taylor approximant $E_{T_{4}}(v)=\sum_{k=0}^{4} E_{k}(\eta) v^{k}$ reaches a minimum, we find, e.g. that (i) when $m=40 M_{\odot}$ and $\eta=0, F_{T_{4}}=200 \mathrm{~Hz}$, very different from the exact value of 110 $\mathrm{Hz}$, and (ii) when $m=40 M_{\odot}$ and $\eta=1 / 4$, that $F_{\mathrm{LSO}}^{T_{4}}=221.4 \mathrm{~Hz}$, very different from the other predictions $F_{\mathrm{LSO}}^{P_{4}}=143$ $\mathrm{Hz}$ and $F_{\mathrm{LSO}}^{\text {Ref. }}=118.6 \mathrm{~Hz}$. We compare and contrast in Fig. 目 the Newtonian and relativistic behaviours of the wave amplitude and instantaneous frequency $F(t)$ during the last couple of orbits before the LSO. The blow up of $\dot{F}(t)$, i.e. the fact that the slope of $F(t)$ becomes vertical is an effect which is localized in the last part of the last cycle before the LSO. Note also in Fig. 7 that a less localized consequence of this blow up is that the average frequency a few cycles before the LSO is smaller (for a given $F_{\mathrm{LSO}}$ ) in the relativistic case, than in the (unphysical) Newtonian one. Note that the physical origin of the blow up of $\dot{F}$ is that, just before the LSO the 'effective potential' for the radial motion becomes very flat (before having an inflection point at the LSO). In picturesque terms, the radial motion becomes "groundless" at the LSO. Evidently, the blow up of $\dot{F}$ is due to our use of the 'adiabatic' approximation down to the LSO. In reality, radiation reaction will cause a progressive transition between the inspiral and plunge which will modify the evolution of $F(t)$ in the last cycle before the LSO. We shall discuss this issue in detail in a forthcoming paper [43] and subsequently its data analysis consequences.

\section{B. Edge contribution to the non-resonant relativistic $\tilde{h}_{+}(f)$}

As in the Newtonian-like case we decompose $\tilde{h}(f)$ in two contributions, Eqs. (3.12b) and (3.12d). The non-resonant contribution $\tilde{h}_{+}(f)$ will be dominated by the 'edge' contribution to an integral of our usual type Eq. (2.38). Though the problem is similar to the one we have generically solved in Section IIID we cannot apply the results Eqs. (3.16), (3.17), (3.18a $),(3.18 \mathrm{~b})$, because of the limiting hypothesis (iii) mentioned in our introductory discussion Section IID]. Indeed, the problem is that, in the (physically relevant) case of relativistic signals the functions $a(t)$ and $\psi(t)$ are not smooth at the upper edge $t=t_{\mathrm{LSO}}$. Let us see explicitly in what way they violate smoothness there. Let us first define,

$$
e_{1}(\eta) \equiv\left[\frac{d}{d v}\left(\frac{E^{\prime}(v)}{\mathcal{F}(v)}\right)\right]_{v_{\mathrm{LSO}}}
$$

so that near the LSO we may write:

$$
\frac{E^{\prime}(v)}{\mathcal{F}(v)}=e_{1}\left(v-v_{\mathrm{LSO}}\right)+\mathcal{O}\left[\left(v-v_{\mathrm{LSO}}\right)^{2}\right] .
$$


If we were to use the test-mass approximation for the energy function $E^{\prime}(v)$ and the Newtonian (quadrupole) one for the flux function $\mathcal{F}(v)$ this would give

$$
e_{1}^{P_{0}(\mathrm{tm})}(\eta) \simeq \frac{15}{2} \frac{1}{4 \eta} \frac{1}{v_{\mathrm{LSO}}^{8}\left(1-3 v_{\mathrm{LSO}}^{2}\right)^{\frac{3}{2}}} \simeq \frac{27492}{4 \eta} .
$$

We have numerically estimated the function $\bar{e}_{1}^{P 4}(\eta) \equiv 4 \eta e_{1}(\eta)$, when using the $P_{4}$-approximant of [13] in the definition of Eq. (4.15). We find that to a good approximation

$$
4 \eta e_{1}^{P_{4}}(\eta) \equiv \bar{e}_{1}^{P 4}(\eta) \simeq 26091.61194 \exp (-4.474405683 \eta) .
$$

In terms of

$$
\tau \equiv \frac{t_{\mathrm{LSO}}-t}{m}, \tau \geq 0 \text { for } v \leq v_{\mathrm{LSO}}
$$

and using

$$
\tau=-\int_{v}^{v_{\mathrm{LSO}}} d v \frac{E^{\prime}(v)}{\mathcal{F}(v)}=\frac{1}{2} e_{1}\left(v-v_{\mathrm{LSO}}\right)^{2}+\mathcal{O}\left[\left(v-v_{\mathrm{LSO}}\right)^{3}\right],
$$

and Eq. (4.2) for $\phi(v)$, we find the following approximate representation (valid near the LSO) for the phase $\phi(t)$ :

$$
\begin{aligned}
t-t_{\mathrm{LSO}} & =-m \tau \\
\phi(t)-\phi\left(t_{\mathrm{LSO}}\right) & \simeq-2 v_{\mathrm{LSO}}^{3} \tau+\frac{4 \sqrt{2}}{\sqrt{e_{1}}} v_{\mathrm{LSO}}^{2} \tau^{3 / 2} .
\end{aligned}
$$

Note also that Eq. (4.20) gives the following representation for $v(t)$, and therefore for the amplitude $a(t)=\mathcal{C} v^{2}(t)$

$$
\begin{aligned}
v & \simeq v_{\mathrm{LSO}}-\frac{\sqrt{2}}{\sqrt{e_{1}}} \tau^{\frac{1}{2}}, \\
a(t) & \simeq a_{\mathrm{LSO}}\left[1-\frac{2 \sqrt{2}}{\sqrt{e_{1}}} \frac{1}{v_{\mathrm{LSO}}} \tau^{\frac{1}{2}}\right] .
\end{aligned}
$$

We are interested in evaluating the edge contribution to the integral

$$
I=\tilde{h}_{+}(f)=\int_{-\infty}^{t_{\mathrm{LSO}}} d t a(t) e^{i \psi_{f}^{+}}(t)=m \int_{0}^{+\infty} d \tau a(\tau) e^{i \psi_{f}^{+}(\tau)} .
$$

Near the LSO boundary i.e. near the edge $\tau=0$ in the $\tau$-form of the integral, the amplitude behaves as Eq. (4.22b) while the appropriate phase $\psi_{f}^{+}(\tau)$ behaves, from Eqs. 4.21a) and 4.21b as

$$
\psi_{f}^{+}(\tau) \simeq \psi_{f \mathrm{LSO}}^{+}-2 \pi m\left(F_{\mathrm{LSO}}+f\right) \tau+\frac{4 \sqrt{2}}{\sqrt{e_{1}}} v_{\mathrm{LSO}}^{2} \tau^{3 / 2}
$$

where

$$
\psi_{f \mathrm{LSO}}^{+} \equiv \psi_{f}^{+}\left(t_{\mathrm{LSO}}\right)=2 \pi f t_{\mathrm{LSO}}+\phi_{\mathrm{LSO}} .
$$

The appearance of fractional powers of $\tau$ in the expansions Eqs. (4.22a) and (4.22b) show explicitly the violation of the $\mathcal{C}^{\infty}$ property of $a(t)$ and $\psi(t)$ at the edge. We cannot use the integration-by-parts method to evaluate the expansion of $I_{\text {edge }}$. However, we can still use the general method sketched in Section IID. Without rotating explicitly the the $\tau$-contour in the complex plane the edge contribution to $I$ is obtained by inserting the expansions Eqs. (4.22b) and (4.24) in Eq. (4.23) and expanding everything out, except for the main phase, $\psi_{f \mathrm{LSO}}^{+}-2 \pi m\left(F_{\mathrm{LSO}}+f\right) \tau$ which must be kept in the exponent. This yields

$$
I_{\mathrm{edge}}=m a_{\mathrm{LSO}} e^{i \psi_{f \mathrm{LSO}}^{+}} \int_{0}^{\infty} d \tau e^{-i y \tau}\left(1-\frac{2 \sqrt{2}}{\sqrt{e_{1}}} \frac{1}{v_{\mathrm{LSO}}} \tau^{\frac{1}{2}}+\frac{i 4 \sqrt{2}}{\sqrt{e_{1}}} v_{\mathrm{LSO}}^{2} \tau^{\frac{3}{2}}\right)
$$

where, $y \equiv 2 \pi m\left(F_{\mathrm{LSO}}+f\right)$. 
Note that, instead of rotating $\tau$ in the complex plane, we can (equivalently) consider that $y$ possesses a small negative imaginary contribution: $y \rightarrow y-i 0$. The integrals appearing in Eq. (4.26b) are evaluated by the general formula

$$
i_{\alpha}=\int_{0}^{\infty} d \tau e^{-i y \tau} \tau^{\alpha}=\frac{e^{-i \frac{\pi}{2}(\alpha+1)}}{y^{\alpha+1}} \Gamma(\alpha+1) .
$$

This yields finally

$$
\tilde{h}_{+}^{\text {edge }}(f) \simeq \frac{m a_{\mathrm{LSO}} e^{i\left[\psi_{f \mathrm{LSO}}^{+}\right]}}{i y}\left[1+\left(\frac{3}{2} \frac{F_{\mathrm{LSO}}}{F_{\mathrm{LSO}}+f}-1\right) e^{-i \pi / 4} \frac{\sqrt{2 \pi}}{\sqrt{e_{1}}} \frac{1}{v_{L S O} \sqrt{y}}\right] .
$$

The leading contribution $\left(\propto(i y)^{-1}\right)$ to the relativistic result Eq. (4.28) agrees with the leading contribution in Eq. (3.18b). Note that the next-to-leading contribution does not have the same dependence on $f+F_{\mathrm{LSO}}$ as the corresponding term in the non-relativistic result in Eq. (3.18b). In spite of the breakdown of the formal expansion Eq. (3.18b) the fractional correction given by the last term in the bracket of Eq. (4.28) is checked to be numerically small. This check was the main motivation for us to compute $\tilde{h}_{+}^{\text {edge }}$ to next-to-leading order in the relativistic case. The lesson is that the formal blow up of $\dot{F}$ near the LSO has only a small numerical effect on $\tilde{h}_{+}^{\text {edge }}$. This is again a confirmation that our results are robust under a refinement of our knowledge of the signal. We shall further check below that, as in the Newtonian case, $\tilde{h}_{+}^{\text {edge }}$ has only a negligible effect on overlaps.

\section{Improved stationary phase approximation for relativistic signals}

Let us now consider the resonant contribution $\tilde{h}_{-}(f)$, considered in the crucial domain where the stationary point is near the edge $t_{\mathrm{LSO}}$. As before also, the optimal approximants to $\tilde{h}_{-}(f)$ that we can construct are given by different analytical expressions according to the value of $f$. However, we need now to introduce a new definition of the two ranges of frequencies in which one must (minimally) divide the $f$-axis. More precisely, we introduce a frequency $f_{\text {up }}$, near but below $F_{\max }$, and we shall construct a "lower" approximation $\tilde{h}_{-<}(f)$ in the range $f<f_{\text {up }}$, and an "upper" one $\tilde{h}_{->}(f)$ in the range $f>f_{\text {up }}$ (which includes $f=F_{\max }$ ). The optimal value of $f_{\text {up }}$ will be determined below.

In the lower range, $f<f_{\text {up }}$, we can draw on the work of Sec. [IID. Indeed, in that range there exists a saddle-point in the domain of integration. However, as that saddle-point can become rather near $t_{\text {max }}$ (because $f_{\text {up }}$ is near $F_{\max }$ ), we can significantly improve the usual SPA estimate by using our previous result, i.e. by defining

$$
\begin{aligned}
f \leq f_{\mathrm{up}}: \quad \tilde{h}_{-<}^{\mathrm{irspa}}(f) & =\mathcal{C}\left(\zeta_{<}(f)\right) \frac{a\left(t_{f}\right)}{\sqrt{\dot{F}\left(t_{f}\right)}} e^{i\left[\psi_{f}\left(t_{f}\right)-\pi / 4\right]}, \\
\zeta_{<}(f) & =-\sqrt{\psi_{f}\left(t_{f}\right)-\psi_{f}\left(t_{\max }\right)}
\end{aligned}
$$

The label 'irspa' in Eq. 4.29a stands for improved relativistic SPA.

Let us finally explore the optimal analytic approximation to $\tilde{h}_{-}(f)$ in the upper range $f \geq f_{\text {up }}$. Proceeding as in Section IVB in this case one has

$$
\psi_{f}^{-}(t) \simeq \psi_{f \mathrm{LSO}}^{-}+2 \pi m\left(F_{\mathrm{LSO}}-f\right) \tau-\frac{4 \sqrt{2}}{\sqrt{e_{1}}} v_{\mathrm{LSO}}^{2} \tau^{3 / 2},
$$

where

$$
\psi_{f \mathrm{LSO}}^{-} \equiv \psi_{f}^{-}\left(t_{\mathrm{LSO}}\right)=2 \pi f t_{\mathrm{LSO}}-\phi_{\mathrm{LSO}} .
$$

We shall use this expansion (which replaces the parabolic approximation Eq. (3.5a) used in the Newtonian case) to evaluate the Fourier integral Eq. (3.28). To this end we must introduce a new special function (characteristic of the relativistic phasing near the LSO) to replace the error function. Let us define the function

$$
g_{\frac{3}{2}}(x) \equiv \int_{0}^{\infty} d \hat{\tau} e^{i\left(3 x \hat{\tau}-2 \hat{\tau}^{3 / 2}\right)},
$$

where the new variable $\hat{\tau}$ is related to $\tau$ by $\tau=\alpha \hat{\tau}$ where 


$$
\alpha=\frac{1}{2} v_{\mathrm{LSO}}^{-4 / 3} e_{1}^{1 / 3} .
$$

In the test mass case corresponding to Eq. (4.17) the value of $\alpha$ is $49.83 /(4 \eta)^{1 / 3}$. The value defined by the $P_{4}$ approximant on the other hand is given by combining Eqs. (2.15) and (4.18). In particular, $\alpha$ equals 30.055 (54.578) for $\eta=0.25(0.1)$ respectively. The index $\frac{3}{2}$ in $g_{\frac{3}{2}}(x)$ alludes to the power $\hat{\tau}^{3 / 2}$ replacing the power $\hat{\tau}^{2}$ in the usual error function, and where the conventional coefficients 3 and 2 have been chosen to simplify some formulas (although they complicate others!). The final result is conveniently written in terms of a variable $x$ given by

$$
x=\frac{2 \pi}{3} \alpha m\left(F_{\mathrm{LSO}}-f\right) .
$$

This improved relativistic SPA is thus written as

$$
f \geq f_{\text {up }}: \tilde{h}_{->}^{\text {irspa }}(f)=m \alpha e^{i \psi_{f}^{\mathrm{LSO}}} a\left(t_{\mathrm{LSO}}\right) g_{\frac{3}{2}}(x) .
$$

Note that $f<F_{\mathrm{LSO}}$ corresponds to $x>0$ (saddle-point domain), while $f>F_{\mathrm{LSO}}$ corresponds to $x<0$ (absence of a saddle-point). Roughly speaking the variable $x(f)$ corresponds to $-\zeta(f)$ of the non-relativistic case, and $g_{\frac{3}{2}}(x)$ is the relativistic analogue of the combination $\mathcal{C}(\zeta) e^{i \zeta^{2}}$ appearing in the previous treatment (see, e.g., Eq. 3.31a $)$ ).

It is useful to summarise some properties of the function $g_{\frac{3}{2}}(x)$ :

$$
\begin{aligned}
& g_{\frac{3}{2}}(0)=\frac{1}{3} \frac{(1-i \sqrt{3})}{4^{1 / 3}} \Gamma\left(\frac{2}{3}\right)=0.284347-0.492503 i, \\
& g_{\frac{3}{2}}(x) \sim \sqrt{\frac{4 \pi x}{3}} e^{i\left(x^{3}-\pi / 4\right)}, x>0, x \gg 1, \\
& g_{\frac{3}{2}}(x) \sim \frac{i}{3 x} ; x<0,-x \gg 1 .
\end{aligned}
$$

By expanding the integrand of $g_{\frac{3}{2}}(x)$ in powers of $x$, and integrating term by term [using the properties of the Euler $\Gamma$-integral after having changed the variable of integration: $\left.\hat{\tau}=e^{-\frac{i \pi}{3}}(u / 2)^{\frac{2}{3}}\right]$, one proves that $g_{\frac{3}{2}}(x)$ is given by the following, everywhere convergent, Taylor-Maclaurin expansion:

$$
g_{\frac{3}{2}}(x)=\frac{2^{1 / 3}}{3} e^{-i \pi / 3} \sum_{n=0}^{n=\infty} \frac{\Gamma\left[\frac{2}{3}(n+1)\right]}{n !}\left(\frac{3 x}{2^{2 / 3}} e^{\frac{i \pi}{6}}\right)^{n} .
$$

With about 300 terms the above series represents $g_{\frac{3}{2}}(x)$ accurately enough for values of $x$ in the range $x \in[-2.3,2.3]$. We used this series to generate the plot of $g_{\frac{3}{2}}(x)$ represented in Fig. 8. Though we do not use it in this paper, note that for $-x \rightarrow \infty$, the following (divergent) asymptotic expansion is also valid:

$$
g_{\frac{3}{2}}(x) \sim-\frac{1}{3 x} \sum_{n=0}^{n=\infty} \frac{\Gamma\left(\frac{3}{2} n+1\right)}{n !}\left[\frac{-2}{(-3 x)^{3 / 2}}\right]^{n} e^{-\frac{i \pi}{4}(n+2)} .
$$

In all our calculations of overlaps we shall define the frequency $f_{\text {up }}$ separating the lower range from the upper range by choosing $x_{\mathrm{up}}=0.36$ as the right hand side of Eq. (4.34). This value is chosen so that at $x_{\mathrm{up}}$ one has a smooth transition from the lower to the upper approximation. We have also checked that the overlaps do not change very significantly for $x_{\text {up }}$ between 0.2 and 0.4 .

In summary our best analytic representation of time-windowed relativistic signals in the Fourier-domain would be defined by combining the P-approximant construction of the functions $E^{\prime}(v), \mathcal{F}(v)$ [13] with the total improved relativistic approximants (irtot) defined as

$$
\tilde{h}^{\text {irtot }}(f)=\tilde{h}_{-}^{\text {irspa }}+\tilde{h}_{+}^{\text {edge }},
$$

where $\tilde{h}_{+}^{\text {edge }}$ is defined in Eq. 4.28) and $\tilde{h}_{-}^{\text {irspa }}$ is defined for $f \leq f_{\text {up }}$ by Eqs. (4.29a), 4.29b), and, for $f \geq f_{\text {up }}$ by Eq. (4.35). Actually, as in the case of Newtonian signals, we have found that the inclusion of $\tilde{h}_{+}^{\text {edge }}$ has only a minimal (though favorable) effect on overlaps. Moreover, such a contribution is absent in the case of real signals. Therefore, our final practical and best proposal consists in using only $\tilde{h}_{-}^{\text {irspa }}$ (For simplicity we henceforth drop the subscript minus). We shall henceforth refer to the improved frequency domain stationary phase P-approximants based on the irSPA as the SPP approximants. 


\section{Comparison between the usual SPA, the improved relativistic SPA and the 'exact' SPA (numerical DFT)}

In this Section, we test the accuracy of our analytical approximations in various ways. Fig. 9 compares an inspiral wave from a $(20,20) M_{\odot}$ binary generated by three different methods: (i) directly in the time-domain and terminated when the instantaneous gravitational wave frequency reaches the value at the LSO (solid line), (ii) in the Fourier domain using the usual SPA but with a square window between $f_{\min }=40 \mathrm{~Hz}, f_{\max }=F_{\mathrm{LSO}}$ (uSPAw) and then inverse Fourier transformed (dashed line) and (iii) again in the Fourier domain but using irSPA with Fourier components computed up to Nyquist frequency and then inverse Fourier transformed to obtain its time-domain representation (dotted line). We only exhibit the comparison near the crucial LSO region [Much before the LSO the uSPA is nearly equivalent to the irSPA and they both do a good job in representing the actual signal]. We observe that the uSPAw begins to get out of phase with the wave directly generated in the time-domain during the last cycle and rings a few times beyond the shut-off point. Our new proposal, irSPA, keeps in phase with the time-domain signal until the last moment although it too has a couple of low amplitude cycles beyond the LSO.

Matched filtering involves not just the correlation of two signals but rather their weighted correlation - the weight coming from the detector spectral noise density. To further compare and contrast our new $f$-domain approximants to the usual frequency-windowed SPA it is conceptually useful to compare various approximations in the 'whitened-timedomain' introduced in Section II A above. As discussed above, in this picture (and only in this picture) the optimal filter consists of correlating the output of the detector with an exact copy of the expected signal. The whitened [i.e., convolved with the whitening kernel $w_{\frac{1}{2}}$ Eq. (2.10)] signals are plotted and compared in Fig. 10 which is the same as

Fig. 9 except that all the waves here are whitened (i.e. divided by $\sqrt{S_{n}(f)}$ and then inverse Fourier transformed). The inset in Fig. 10 shows the full whitened signal that was originally generated in the time domain. Several observations are in order. First, we see how low frequency components are suppressed relative to high frequency components which occur in a more sensitive band of the detector. Second, we can very clearly see the non-local behaviour of the whitening kernel. It has the effect of softening the window imposed on the wave that was directly generated in the time-domain and curbing the oscillations in the irSPA beyond $F_{\text {LSO }}$. Finally, this same whitening is seen to have worsened the mismatch of USPAw with the whitened version of the original time-truncated signal. The conclusions drawn from these visual comparisons are borne out by detailed numerical experiments we performed.

To compare the approximants more quantitatively, in Table III we list the overlaps of the exact Fourier representation of a model waveform (i.e., a signal generated in the time-domain and then Fourier transformed using a DFT algorithm) with their approximate Fourier representations analytically computed using one of the following: the frequencywindowed usual SPA [i.e., uSPAw, cf.Eq. (3.7)], the improved Newtonian SPA [inSPA is the same as irSPAw, cf. Eq. (4.29b)] and the improved relativistic SPA [cf. Eq. (4.39)]. The uSPA and the inSPA used in computing these overlaps are terminated at $f=F_{\mathrm{LSO}}^{\mathrm{A}}$, where $F_{\mathrm{LSO}}^{\mathrm{A}}$ is the last stable orbit frequency determined by the condition $E_{\mathrm{A}}^{\prime}(v)=0$ (hence the labels SPAw and inSPAw where 'w' stands for 'windowed' — in the frequency-domain). This is because both uSPA and inSPA vanish at the LSO (due to the factor $1 / \sqrt{\dot{F}_{\mathrm{LSO}}}$ ) and are either not defined (in the case of the usual SPA) or formally vanishing (according to the definition Eq. (3.31a) in the case of inSPA) beyond the LSO. Contrast this with the Newtonian case where it is possible to analytically extend the usual SPA beyond $F_{\text {LSO }}$.

It is generally true, as stated in DIS, that the stationary phase approximation to the Fourier transform worsens very significantly as we consider more massive binaries. In this sense the uSPA poorly represents the exact chirp. We conclude that, for massive systems with total mass $m=m_{1}+m_{2} \lesssim 40 M_{\odot}$ the only uniformly acceptable analytic representation of the Fourier transform is the irSPA.

\section{FAITHFULNESS AND EFFECTUALNESS OF SPP APPROXIMANTS}

So far we have concentrated on developing an accurate Fourier representation of the inspiral waveform at various levels of approximation from Newtonian to P-approximants. In order to quantify the accuracy, we used the overlap of the DFT of the waveform computed using a FFT of the time-domain signal with an analytical approximation of the Fourier transform of the same time-domain signal using the improved SPA suggested in Sec. III and IV. However, an important question still remains: What is the total loss of accuracy due to combining the loss of precision entailed by the use of an analytical approximant to the FT (loss that we have shown how to minimize by defining the irspa) with the loss of accuracy $y^{10}$ entailed by the use of some finite-order in the post-Newtonian approximation of the exact signal.

${ }^{10}$ We distinguish precision and accuracy in the same way that they are distinguished in Metrology. 
In other words, how accurate is the approximate frequency-domain representation of a post-Newtonian approximant in modelling the exact FT of the exact general relativistic signal? More precisely, what fraction of the SNR of a true signal is the Fourier-domain approximant likely to extract? Additionally, one is also interested in knowing the biases induced in the estimation of parameters when using the frequency-domain approximants introduced in this work.

We shall follow DIS in saying that a representation of a signal is faithful if it has a good overlap with the exact signal for the same values of the (dynamical) parameters (or more precisely, if the overlap is maximized for template parameters which have acceptably small biases with respect to the exact signal parameters). As in DIS, we employ as necessary criterion for faithfulness the requirement that the 'diagonal' ambiguity function be larger than 0.965 . On the other hand, we shall say that a representation of a signal is effectual if the overlap, maximized over the template parameters is very near one. To use these definitions we follow DIS in introducing a fiducial exact general relativistic signal. In Table IV and Table VI we use as fiducial exact signal the formal "test-mass case" for which the function $E(v)$ is known analytically and $\mathcal{F}(v)$ numerically [26]. In Table $\mathbb{Q}$ we use as fiducial exact signal the one defined in DIS for comparable masses [see Eq. (4.11) there for the definition of the exact new energy function, and Eqs. (7.1) (7.2) for the exact factored flux function; we took the value $\kappa_{0}=47 / 39$ for the parameter defining formal higher PN-effects in Eq. (4.11) ]. As above we consider that the exact time-domain signal is shut off after the LSO. [For each considered waveform, defined by some approximate energy and flux functions $E_{A}(v)$ and $\mathcal{F}_{A}(v)$, we shut it off at the LSO defined by the corresponding energy function $\left.E_{A}(v).\right]$

In Tables [V] and $\mathrm{V}$ we list the overlaps for different approximants for the three 'massive' archetypal binaries $\left[\left(1.4 M_{\odot}, 10 M_{\odot}\right),\left(10 M_{\odot}, 10 M_{\odot}\right)\right.$, and $\left.\left(20 M_{\odot}, 20 M_{\odot}\right)\right]$ that could be searched for in GEO/LIGO/VIRGO data. These overlaps are computed using the expected LIGO noise Eq. (1.4a) by maximising over the lag parameter $\tau$ [47] and phase $\phi_{c}$ but without re-adjusting the intrinsic parameters i.e., the masses of the two stars in the approximants, to maximise the overlap. [This implies that the value of $F_{\mathrm{LSO}}$ used in the approximant is different from that in the 'exact' signal.] The overlaps are therefore a reflection of how accurate the various representations are in an absolute sense. In other words, they compare the faithfulness of the different approximants. Two independent aspects of approximation are investigated in these Tables. Firstly, the comparison between the two alternatives in the frequency domain: the usual SPA (uspaw) and our improved relativistic SPA (irspa). And secondly, the post-Newtonian order to which the phasing is computed. To investigate further the performance of these approximants we summarise in Table VI the overlaps obtained by maximising over all the parameters in the approximants including the intrinsic ones. Thus in addition to maximising over the lag parameter $\tau$ and the phase $\phi_{c}$ one also extremises over the masses of the two stars $m_{1}$ and $m_{2}$. In other words, we compare the effectualness of the various approximants. We also compute the bias introduced in the total mass $m$.

From Tables IV- VI one can conclude the following: (i) The improved relativistic SPA is significantly more faithful and more effectual for massive systems with total mass $m \gtrsim 20 M_{\odot}$, and mandatory for $m \gtrsim 26 M_{\odot}$, (ii) Comparing with DIS, we see that the frequency-domain irspa does as well as the time-domain waveform even for massive binaries up to $40 M_{\odot}$; (iii) The 2.5PN SPP approximant is both a faithful and an effectual approximant for a wide range of binary systems $\left(m \lesssim 40 M_{\odot}\right)$. It only introduces a small bias. Note also that, in regard to effectualness, the gain in going from $2 \mathrm{PN}$ to $2.5 \mathrm{PN}$ accuracy is quite significant (mainly in decreasing the biases) and especially for low-mass systems (which have many useful cycles), while the gain in going from $2.5 \mathrm{PN}$ to $3 \mathrm{PN}$ seems very slight.

To summarise: If one would like to lose no more than a tenth of the events that would be observable had one known the exact general-relativistic signal, then the 2.5PN SPP-approximants are a must. Furthermore, unbiased parameter estimation requires 2.5PN SPP-approximants in all cases.

\section{WHY ARE TIME DOMAIN RELATIVISTIC SIGNALS MORE EXPENSIVE TO COMPUTE?}

The main purpose of this work is to provide a set of tools to the experimenters so that they can generate templates with a minimal computational cost. We next, therefore, address the issue of computational costs of various algorithms for template generation.

First, though the signal is initially given in the time-domain, the time-domain version of the Wiener filter contains a double time integration [see second form of Eq. (2.2)] which is (given the existence of FFT algorithms) much more computationally expensive than the single frequency-domain version of the Wiener filter [see first form of Eq. (2.2)]. Therefore, in the computation of the correlation of a template with the detector output what is required is the Fourier

\footnotetext{
${ }^{11}$ When discussing faithfulness and effectualness we always assume, as in DIS, Eq 2.17 there, that the overlap Eq. (2.8) is first maximized with respect to the relative time lag (and relative phase).
} 
transform of the matched filter. However, the DIS proposal was to compute the templates in the time-domain and compute their exact DFT using FFT algorithms. Admissibly, this procedure is still highly computation-intensive. Let us reason out why this is so.

To compute the time-domain signal we need a phasing formula $\phi=\phi(t)$. Since there is no explicit expression for the phasing of inspiral waves as a function of time the standard approach is to use the implicit formula, Eqs. (4.1)-(4.2). The binding energy $E(v)$ and the gravitational wave flux $\mathcal{F}(v)$ have been computed, e.g. using Padé techniques, as explicit functions of $v$ and these when used in Eq. (4.1) and (4.2) yield an implicit relation between $\phi$ and $t$. However, the problem is that we need $\phi$ at equal intervals of time (to enable us to use the standard FFT algorithms) and this makes the computation of $\phi(t)$ expensive: every time-sample $\phi_{i} \equiv \phi\left(t_{i}\right)$ is computed by first solving Eq. (4.1) iteratively for $v_{i}$, the lower limit in the integral for a given $t_{i}$, and then using this $v_{i}$ as the lower limit in the integral of Eq. (4.2). Though the second step is the computation of a single integral, the first step is a rather slowly converging $\left(\sim 10\right.$ iterations for every $\left.t_{i}\right)$ computation.

This problem could have been circumvented if it had been adequate to use the explicit analytical expression $\phi(t)=b_{0}\left(t_{\mathrm{LSO}}-t\right)^{5 / 8}+\sum_{k \geq 1} b_{k}\left(t_{\mathrm{LSO}}-t\right)^{(4-k) / 8}$ (modulo logarithms) obtained by : (i) expanding the quantity $E^{\prime}(v) / \mathcal{F}(v)$ in the integrands, in a straightforward expansion in powers of $v$, (ii) integrating term by term, and (iii) inverting analytically by successive iterations (see e.g. 24]). However, this straightforward PN expansion of the phasing formula defeats the very purpose of P-approximants and loses all the benefits brought by the constructions given in [13]. Consequently, DIS had to use the iterative procedure to compute the signal phasing. By contrast, using (any form) of SPA, i.e. an explicit analytical f-domain expression, brings a tremendous reduction in computational costs. On the one hand, as we shall discuss below there is no iterative procedure involved in computing SPA. Secondly they are computed directly in the frequency-domain and hence lead to a further cost reduction, since time-domain waveforms need to be Fourier transformed using FFTs - $\operatorname{costing} N \log _{2} N$ floating point operations — in addition to floating point operations required to compute time-domain templates.

Let us recall that the usual SPA is given by Eq. (3.7). In this expression $t_{f}$ is the stationary point of the phase in the integral of Eq. (3.4b). At a Fourier frequency $f=v_{f}^{3} / \pi m$ the stationary point $t_{f}$ is given by Eq. (4.5), which is a non-iterative computation. One then substitutes this value of $t_{f}$ in Eq. (4.6) to compute $\psi_{f}$ - the phase of the Fourier component. Moreover, the derivative of the frequency which occurs in the amplitude of the Fourier transform can be computed using Eq. (4.14) while the factor $a\left(t_{f}\right) \propto f^{2 / 3}$ from Eq. (3.2a). Every quantity that appears in the SPA is computed using a straighforward integral or a mere algebraic expression. Hence, from the computational-cost point-of-view, it is desirable to use some SPA to generate templates. Since the usual SPA has been shown to be inadequate for representing time-windowed signals from massive binaries, we have proposed the use of corrections to (for $f \leq f_{\text {up }} \leq F_{\mathrm{LSO}}$ ) and analytic extensions of (for $f \geq F_{\text {up }}$ ) the usual SPA. In Table VII we compare for archetypal binaries, the computational costs of templates that are generated in the time-domain and Fourier transformed using an FFT algorithm with the computational costs for the USPA, inSPA and irSPA. This Table clearly shows that it is sensible to generate templates in the Fourier domain. The SPA is up to a factor 100 times faster and the irSPA is up to a factor 10 times faster than the corresponding time-domain construction and Fourier transformation. Table VII together with Table V (of overlaps) demonstrates that SPP approximants while more expensive to generate than the usual SPA are nevertheless 'affordable', and are anyway necessary for efficient searches of inspiral signals in gravitational wave interferometer data.

\section{CONCLUDING REMARKS}

After nearly two decades of detector-technology development long-baseline interferometric gravitational wave antennas LIGO/VIRGO are scheduled to become operational in about 2-4 years with target sensitivities that are good enough to detect inspiral events from massive $\left(m>20 M_{\odot}\right)$ binaries at an optimistic rate of a few per year. Searches are planned to be carried out over a range of $0.2-50 M_{\odot}$ by the method of matched filtering.

An important issue in matched filtering is the number of cycles accumulated in the correlation integral since the SNR grows as the square-root of the number of cycles. While this is strictly true, if the noise power spectrum of the instrument is independent of frequency, in practice one can only improve the SNR in proportion to the square-root of a "useful" number of cycles $N_{\text {useful }}$ which is determined by a combination of the detector noise power spectrum and the signal's power-spectrum. We have pointed out how the number of useful cycles can be a lot smaller than the actual number of cycles for massive and relativistic systems: e.g. a $\left(10 M_{\odot}, 10 M_{\odot}\right)\left[\left(20 M_{\odot}, 20 M_{\odot}\right)\right]$ binary system has only 7.6 [3.4] useful cycles in the detector's bandwidth (see Table Ii). A priori, it may seem that the fewer number of cycles should make it easier to model the massive black hole binaries compared to the lighter neutron star-neutron star ones with its corresponding large number of cycles to phase. Tables IV VI show that there is some truth in this, but that for very massive black hole binaries, these fewer cycles are in fact more difficult to model than the neutron 
star-neutron star, or neutron star-black hole cases for two reasons: (i) they are near the end of the inspiral, i.e. when the radiation reaction effects drives a faster drift of the frequency which has to be modelled accurately (this is why we need P-approximants introduced in DIS); (ii) they might terminate due to the transition from inspiral to plunge while in the detector's bandwidth, and this poses the problem of accurately describing the Fourier transform of a time-windowed signal (this requires the correction factors introduced in this paper). All this places stringent demands in modelling the waveform in the Fourier-domain and due attention needs to be paid to delicate issues of detail. This task is all the more important that the first detections expected from LIGO/VIRGO are likely to concern massive systems with $m \sim 25 \pm 5 M_{\odot}$, for which the LSO frequency lies near the middle of the sensitivity curve [see Fig. 11].

To this end, the present work makes two new robust (i.e. assumption-independent) contributions:

- the proposal of stationary phase P-approximants (SPP) which combine the excellent performance of our timedomain P-approximants [13] with the analytic convenience of the stationary phase approximation without serious loss of event-rate. These Fourier-domain P-approximants perform as well as their time-domain counterparts in extracting the true general relativistic signal.

- the definition of a universal Newtonian-like 'edge-correction' factor $\mathcal{C}(\zeta(f)$ ), as well as its relativistic complement $g_{\frac{3}{2}}(x(f))$ which take into account the frequency-domain effects, concentrated around (and on both sides) of $F_{\max }=F\left(t_{\max }\right)$, for signals which are abruptly shut off, in the time-domain, after $t_{\max }$.

In addition to these new achievements, let us mention two other useful contributions, of a more technical nature: (i) our recommendation to systematically use a smooth time window at the lower frequency side to conveniently and efficiently suppress spurious oscillations due to a numerical low frequency cutoff and (ii) the emphasis on the comparison of the form of the signals in the 'whitened' time-domain.

Based on the detailed analysis presented in this paper we find that for post-Newtonian template generation of binary systems of total mass $m \lesssim 5 M_{\odot}$ it suffices to use the usual SPA (without correction factor) of the P-approximants defined in DIS. On the other hand, in the total mass range $5 M_{\odot} \lesssim m \lesssim 40 M_{\odot}$, it is crucial to use our new SPP approximants to construct the frequency-domain templates.

In addition to the construction of the SPP approximants, the paper has examined in detail the Fourier-domain effects entailed by a sharp time-domain windowing. As emphasized in the introduction, at our present stage of knowledge, one cannot be sure that a template waveform terminated (in the time-domain) at the LSO is an accurateenough representation of a real GW signal coming from massive binaries (say with $m<40 M_{\odot}$ ). We have given several plausibility arguments towards justifying this assumption: brevity of the plunge, and an expected frequency separation from the merger signal. In the absence of knowledge of the transient plunge signal and of the final merger signal, we have argued that it is best to use a template waveform which is terminated at the LSO. [Actually, we anticipate that the effectualness of the template waveform will be increased if we allow it to be terminated at a frequency somewhat larger than $F_{\text {LSO }}$ (thereby allowing it to approximately represent the plunge waveform).] Consequently, this work has concentrated on signal models that are truncated in the time-domain by a step-function and has aimed at constructing the best associated Fourier-domain analytical representation for this possibility.

We have also pointed out that the opposite assumption of an abrupt termination at $F_{\text {LSO }}$ of the usual SPA in the frequency domain implies, when viewed from either the time-domain or the whitened time-domain, the existence of some coherent oscillations 'ringing' after the LSO crossing. We have done another numerical experiment on this issue, by appending to the inspiral signal a smooth decay taking place over less than $3 F_{\text {LSO }}^{-1}$ time-scales. We have found that our improved SPA was a reasonably good representation of (the FT of) such a signal, and definitely a better representation than the usual SPA one. Let us finally reiterate that, we do not claim to have conclusively ruled out the possibility that a frequency-windowed SPA may perform better compared to the time-windowed SPA we propose here. This important issue is not settled though we conjecture that this is unlikely. Anyway, this paper is the first one to explicitly construct the frequency domain version of the time-domain P-approximants which were shown in DIS to bring indispensible improvements over the usually considered T-approximants. Therefore, even in the unlikely case where a straightforward frequency-window turns out to be a better model than the time-window assumed in most of this work, one will still require the formulas given in this paper (with the trivial change of replacing the correction factors $\mathcal{C}(\zeta)$ by a $\theta$ function $\theta\left(F_{\mathrm{LSO}}-f\right)$ ) to generate sufficiently accurate f-domain filters. In view of these comments, we feel there is a urgent need to model more precisely the transition from the inspiral signal to the plunge signal [43] close to the last stable orbit. We hope that the techniques (if not all the details of our construction) used in this work to handle the blow up of $\dot{F}(t)$ at the LSO will be useful (maybe with some modifications) even if, on a later examination, this blow up turns out to be an artefact of an approximation which may drastically alter with a better treatment of the transition to the plunge. Only with this improved understanding and its implications for the construction of templates can one build even more optimal templates for massive binaries and maximise our chance of detecting them. Independently of issues such as windowing in time versus windowing in frequency or the nature of 
the plunge we feel that in general P-approximants are much better tools than the Taylor approximants. We hope to come back to this question in a future work [48].

Another aspect that needs to be looked into is the issue of whether whether the interferometers will work in the time-domain or the frequency-domain. If indeed, they would decide to work in the time domain: i.e., to store the raw output, and to transform it nearly online in the defiltered time-domain equivalent GW amplitude $h(t)$ the analysis of this paper would be irrelevant. In that case, one should store the Wiener transformed time-domain filter $K(t)=w_{1} * h(t)$. However, with the presently available computational resources it seems hopeless to filter in the time-domain. We therefore anticipate that, though the raw detector output will be stored in the time-domain, all filtering will be done in the Fourier domain. In this event, the robust aspects of the present analysis will be relevant even if not the details.

The formalism developed in this paper can be applied not only to initial interferometers but also to future generations of interferometers. We have refrained from applying our formalism to the case of LIGO II since the LIGO II design is at the moment in a state of flux and any quantitative results we may quote will soon be irrelevant. However, we should expect the results of this work to be important for any detector that works with a lower seismic cutoff and a broader bandwidth than LIGO I, since in such cases we will have to match the signal's phase for a larger number of effective cycles.

There are several notable and obvious improvements that need to be pursued. The sensitivity to the value of $F_{\mathrm{LSO}}$ needs to be investigated [in particular, our improved SPA will probably maximize their overlaps with the real signals if we allow some flexibility in the choice of $F_{\text {LSO }}$ (within some limits)]. Once the results of 3PN generation of gravitational waves are available [49] and are combined with the 3PN results on the dynamics [41] they must be included in the construction of templates. In our discussion we have not considered waveforms from binaries with spinning compact objects. Nor have we included the effect of eccentricity [50] on the detectability [23]. These are unarguably important physical effects that need to be incorporated in later data analysis algorithms. Future research in this area should shed light on these issues.

\section{ACKNOWLEDGMENTS}

We thank J.Y. Vinet for informative communications concerning the VIRGO noise curve. BRI and BSS thank A. Gopakumar and B.J. Owen for discussions on the validity of the stationary phase approximation. BRI would like to thank AEI, Germany and PPARC, U.K. for visiting fellowships.

\section{APPENDIX A: LIST OF SYMBOLS}

\begin{tabular}{|c|c|}
\hline$a(t)$ & GW amplitude; $h(t)=2 a(t) \cos \phi(t)$ \\
\hline$\alpha$ & $=\frac{1}{2} v_{\mathrm{LSO}}^{-4 / 3} e_{1}^{1 / 3} ; \mathrm{Eq} .(4.33)$ \\
\hline cspa & corrected SPA; Eqs. (3.29a $-(3.29 \mathrm{~b})$ \\
\hline$C_{n}\left(t_{1}-t_{2}\right)$ & correlation function of noise \\
\hline $\mathcal{C}(\zeta)$ & $\begin{array}{l}\frac{1}{2} \operatorname{erfc}\left(e^{i \pi / 4} \zeta\right) ; \text { correction factor; softened step function } \\
\text { leading phase correction to SPA; Eq. }\end{array}$ \\
\hline$\eta$ & symmetric mass ratio $\equiv m_{1} m_{2} /\left(m_{1}+m_{2}\right)^{2}$ \\
\hline $\operatorname{erfc}(x)$ & complementary error function; Eq. (3.21) \\
\hline$e_{1}(\eta)$ & $\equiv\left[\frac{d}{d v}\left(\frac{E^{\prime}(v)}{\mathcal{F}(v)}\right)\right]_{v_{\mathrm{I} S O}} ; \mathrm{Eq} \cdot(4.15)$ \\
\hline$E(v)$ & dimensionless energy function \\
\hline$\varepsilon_{1}$ & $\equiv\left|\frac{\dot{a}(t)}{a(t) \dot{\phi}(t)}\right| ; \mathrm{Eq} .(3.8)$ \\
\hline$\varepsilon_{2}$ & $\equiv\left|\frac{\ddot{\phi}(t)}{\dot{\phi}^{2}(t)}\right|=\left|\frac{1}{2 \pi} \frac{\dot{F}(t)}{F^{2}(t)}\right|=\frac{1}{2 \pi N} ;$ Eq. 3.8 \\
\hline FFT & Fast Fourier transform \\
\hline f-domain & frequency-domain \\
\hline f-window & frequency window \\
\hline$F(t)$ & instantaneous GW frequency \\
\hline $\mathcal{F}(v)$ & flux function \\
\hline$F_{\min }\left(F_{\max }\right)$ & GW frequency at $t_{\min }\left(t_{\max }\right)$ \\
\hline$F_{\mathrm{LSO}}$ & GW frequency at LSO \\
\hline$F_{\text {Nyquist }}$ & Nyquist Frequency \\
\hline
\end{tabular}




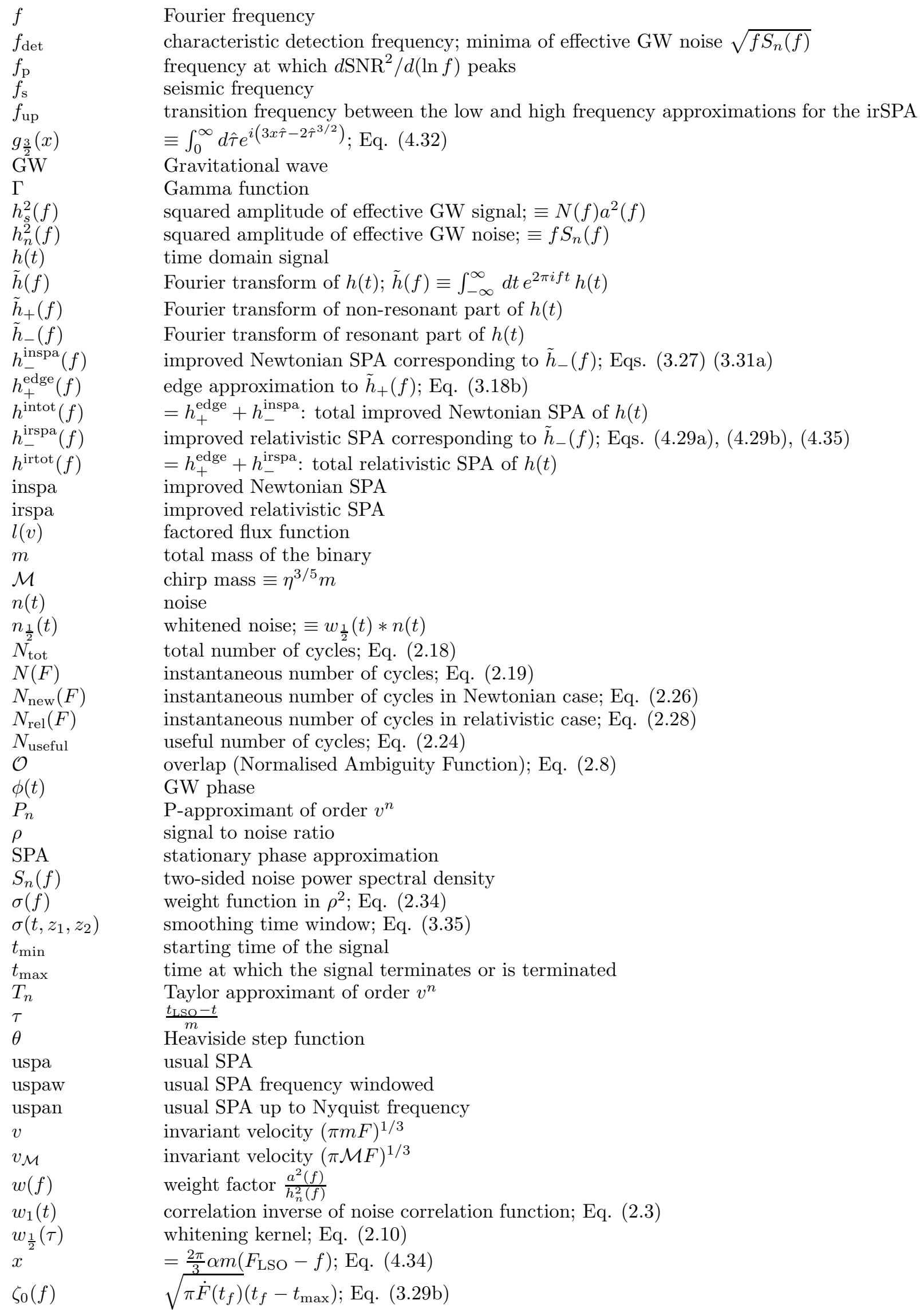


[1] R.A. Hulse and J.H. Taylor, Astrophys. J. Lett., 195, L51 (1975).

[2] See e.g. 1993 Nobel lectures in physics of R.A. Hulse (pp. 699-710) and J.H. Taylor (pp. 711-719) in Rev. Mod. Phys. 66 (1994).

[3] T. Damour, in Gravitational Radiation, ed. N. Deruelle and T. Piran, pp 59-144 (North-Holland, Amsterdam, 1983) and references therein.

[4] J. H. Taylor, A. Wolszczan, T. Damour and J. M. Weisberg, Nature, 355, 132 (1992).

[5] K.S. Thorne, in 300 Years of Gravitation, edited by S.W. Hawking and W. Israel (Cambridge University Press, Cambridge, England, 1987), p. 330.

[6] B.F. Schutz, in The Detection of Gravitational Waves, edited by D.G. Blair (Cambridge University Press, Cambridge, England, 1991), p. 406.

[7] H. Lück et al., Class. Quantum Grav. 14, 1471 (1997).

[8] A. Abramovici et al., Science 256, 325 (1992).

[9] B. Caron et al., Class. Quantum Grav. 14, 1461 (1997).

[10] B.S. Sathyaprakash and S.V. Dhurandhar, Phys. Rev. D 44, 3819 (1991); S.V. Dhurandhar and B.S. Sathyaprakash, Phys. Rev. D 49, 1707 (1994); R. Balasubramanian and S.V. Dhurandhar, Phys. Rev. D 50, 6080 (1994).

[11] B.S. Sathyaprakash, Phys. Rev. D 50, 7111 (1994); B.J. Owen, Phys. Rev. D 53, 6749 (1996); B.J. Owen and B.S. Sathyaprakash, Phys. Rev. D 60, 022002 (1999).

[12] S. Droz and E. Poisson, Phys. Rev. D 56, 4449 (1997); S. Droz, Phys. Rev. D 59, 064030 (1999).

[13] T. Damour, B.R. Iyer, and B.S. Sathyaprakash, Phys. Rev. D 57, 885 (1998). It is referred to as DIS in this paper.

[14] We stress the need to be careful when comparing qualitative statements in publications by different authors regarding the PN order adequate for detection or the necessity or otherwise of different approximants to the waveform. For the user, as always, the fine print regarding the choice of various parameters is equally important: e.g. some papers 12 consider $90 \%$ overlap acceptable (though it corresponds to a loss of $27 \%$ in potential events), others 13] insist on considering $96.5 \%$ (only 10\% loss); some papers actually refer to Advanced LIGO which may be irrelevant for initial LIGO; claims may refer only to lighter binaries rather than be a comprehensive one including massive binaries.

[15] C. Cutler and É.É. Flanagan, Phys. Rev. D 49, 2658 (1994).

[16] E. Poisson and C.M. Will, Phys. Rev. D 52, 848 (1995).

[17] A. Krolak, K. Kokkotas and G. Schäfer, Phys. Rev. D 52, 2089 (1995).

[18] R. Balasubramanian, B.S. Sathyaprakash, and S.V. Dhurandhar, Phys. Rev. D 53, 3033 (1996); R. Balasubramanian and S.V. Dhurandhar, Phys. Rev. D 57, 3408 (1998); D. Nicholson and A. Vecchio, Phys. Rev. D 57, 4588 (1998).

[19] B.F. Schutz, Nature, 323, 310 (1986); D.F. Chernoff and L.S. Finn, Ap. J. 411, L5 (1993); L.S. Finn and D.F. Chernoff, Phys. Rev. D 47, 2198 (1993).

[20] L. Blanchet and B.S. Sathyaprakash, Class. Quantum Grav., 11, 2807 (1994); Phys. Rev. Lett., 74, 1067 (1995).

[21] S. Mohanty and S.V. Dhurandhar, Phys. Rev. D 54, 7108, (1996); S. Mohanty, Phys. Rev. D 57, 630, (1998).

[22] T.A. Apostolatos, Phys. Rev. D 52, 605 (1996); Phys. Rev. D 54, 2421 (1996).

[23] K. Martel and E. Poisson, gr-qc/9907006.

[24] L. Blanchet, T. Damour, B.R. Iyer, C.M. Will and A.G. Wiseman, Phys. Rev. Lett. 74, 3515 (1995); L. Blanchet, T. Damour and B.R. Iyer, Phys. Rev. D51, 5360 (1995); C.M. Will and A.G. Wiseman, Phys. Rev. D54, 4813 (1996); L. Blanchet, B.R. Iyer, C.M. Will and A.G. Wiseman, Class. Quantum. Gr. 13, 575, (1996); L. Blanchet, Phys. Rev. D54, 1417 (1996).

[25] C. Cutler et al., Phys. Rev. Lett. 70, 2984 (1993).

[26] E. Poisson, Phys. Rev. D47, 1497 (1993).

[27] E. Poisson, in Second Workshop on Gravitational Wave Data Analysis, Eds. M. Davier and P. Hello, 107, (1998), Edition Frontières

[28] B.S. Sathyaprakash in Laser Interferometer Space Antenna, Ed. W.M. Folkner, AIP (1998).

[29] K.S. Thorne [5]; S.V. Dhurandhar, A. Krolak and B. Schutz and J. Watkins, (unpublished preprint); See also B.S. Sathyaprakash and S.V. Dhurandhar in 10$]$.

[30] E. Chassande-Mottin and P. Flandrin, Proc. IEEE Symposium on Time Frequency and Time Scale Analysis, 117 (1998).

[31] S. Droz, D.J. Knapp, E. Poisson and B.J. Owen, Phys. Rev. D 59, 124016 (1999).

[32] We use the functional form used in reference 15. The value of $S_{0}$ is read off using the curves in K.S. Thorne, in Proceedings 
of the Snowmass 95 summer study on particle and nuclear astrophysics, Eds. E.W. Kolb and R. Peccei (World Scientific, Singapore, 1995) gr-qc 9506086; and B.F. Schutz, in, Relativistic Gravitation and Gravitational Radiation, Eds. J.A. Marck, J.P. Lasota. Cambridge Univ. Press (1997), 447-475.

[33] M. Beccaria, E. Cuoco et G. Curci, VIRGO Internal Note, VIR-NOT-PIS-1390-096, (1996).

[34] A.M. Cherepashchuk, Uspekhi Fiz. Nauk. bf 166, 809 (1996); Y. Tanaka and N. Shibazaki, Ann. Rev. Astron. Ap. 34607 (1997).

[35] V.M. Lipunov, K.A. Postnov and M.E. Prokhorov, New Astron. 243 (1997).

[36] E.E. Flanagan and A.A. Hughes, Phys. Rev. D57, 4535 (1997).

[37] P.R. Brady, J.D.E. Creighton and K.S. Thorne, Phys. Rev. 58, 061501 (1998).

[38] K.A. Postnov and M.E. Prokhorov, Proceedings of the 34th Moriond Conference on Gravitational waves and relativistic gravity, astro-ph 9903193.

[39] E. Poisson, Phys. Rev. D47, 1497 (1993); C. Cutler, L.S. Finn, E. Poisson and G.J. Sussmann, Phys. Rev. D47, 1511 (1993); H. Tagoshi and T. Nakamura, Phys. Rev. D49, 4016 (1994); M. Sasaki, Prog. Theor. Phys. 92, 17 (1994); H. Tagoshi and M. Sasaki, Prog. Theor.Phys. 92, 745 (1994); T.Tanaka, H.Tagoshi and M.Sasaki, Prog. Theor. Phys. 96, 1087 (1996).

[40] A. Buonanno and T. Damour, Phys.Rev. D 59, 084006 (1999); gr-qc/9811091.

[41] P. Jaranowski and G. Schäfer, Phys. Rev. D57, 7274 (1998); T. Damour, P. Jaranowski and G. Schäfer, gr-qc 000 3051; L. Blanchet and G. Faye gr-qc 000 4008, grqc 000 4009; M. Pati and C.M. Will, (Work in progress).

[42] T. Damour, P. Jaranowski and G. Schäfer, gr-qc 9912 092; (Work in progress).

[43] A. Buonanno and T. Damour, gr-qc 0001013

[44] S. Chandrasekhar and S. Detweiler, Proc. Roy. Soc. A 344, 441 (1975).

[45] C.M. Bender and S.A. Orszag, Advanced Mathematical Methods for Scientists and Engineers (McGraw Hill, New York, 1978) Chap. 6.

[46] Consider two signals $q_{1}$ and $q_{2}$ with supports $S_{1}=\left[f_{\min }, f_{\max }\right]$ and $S_{2}=\left[f_{\min }, f_{\mathrm{Ny}}\right]$ with $f_{\mathrm{Ny}}>f_{\max }$. Even when $q_{1}$ and $q_{2}$ perfectly match with each other in $S_{1}$ (both in amplitude and phase) their overlap will in general be less than 1 . Indeed, $\left\langle q_{1}, q_{2}\right\rangle=\left\|q_{1}\right\| /\left\|q_{2}\right\|$, where $\left\|q_{i}\right\| \equiv\left\langle q_{i}, q_{i}\right\rangle^{1 / 2}, i=1$, 2. Since $S_{1} \subset S_{2},\left\|q_{1}\right\| \leq\left\|q_{2}\right\|$. This explains why uSPAn returns less overlaps than uSPAw.

[47] In the case where one introduces a time lag $\tau$ between two signals the Wiener scalar product reads $\langle h, g\rangle\left(\tau ; \lambda_{k}, \mu_{k}\right) \equiv$ $\int_{-\infty}^{\infty} \frac{d f e^{2 \pi i f \tau}}{S_{n}(f)} \tilde{h}^{*}\left(f ; \lambda_{k}\right) \tilde{g}\left(f ; \mu_{k}\right)$, where ${ }^{*}$ denotes complex conjugation, $\lambda_{k}, k=1, \ldots, n_{\lambda}$, and $\mu_{k}, k=1, \ldots, n_{\mu}$, are the parameters of the signals and $n_{\lambda}$ and $n_{\mu}$ are the corresponding number of parameters 13 .

[48] T. Damour, B.R. Iyer and B.S. Sathyaprakash, (Work in progress).

[49] L. Blanchet, B.R. Iyer and B. Joguet, (Work in progress).

[50] A. Gopakumar and B.R. Iyer, Phys. Rev. D56, 7708 (1997). 
TABLE I. Number of useful cycles for some representative systems and approximants. For each of the cases the corresponding total number of cycles is listed within rounded brackets in the line below.

\begin{tabular}{|c|c|c|}
\hline System & Newtonian & Relativistic: $P_{4}$ \\
\hline$\overline{1.4 M_{\odot}-1.4 M_{\odot}}$ & $\begin{array}{c}173 \\
(1588)\end{array}$ & $\begin{array}{c}169 \\
(1586)\end{array}$ \\
\hline $1.4 M_{\odot}-10 M_{\odot}$ & $\begin{array}{c}51 \\
(348)\end{array}$ & $\begin{array}{c}37 \\
(320)\end{array}$ \\
\hline $10 M_{\odot}-10 M_{\odot}$ & $\begin{array}{c}12 \\
(57)\end{array}$ & $\begin{array}{c}7.6 \\
(47)\end{array}$ \\
\hline $20 M_{\odot}-20 M_{\odot}$ & $\begin{array}{c}9.2 \\
(15)\end{array}$ & $\begin{array}{c}3.4 \\
(10)\end{array}$ \\
\hline
\end{tabular}

TABLE II. Accuracy of the various approximations to the Fourier transform of the chirp signal in the test mass limit for Newtonian waveforms. The accuracy is estimated via the overlap, Eq. (2.8), of a waveform generated in the time-domain and then Fourier transformed using an FFT algorithm, with waveforms of exactly the same parameters generated directly in the frequency domain via one of the following approximations: The usual stationary phase approximation truncated at $f_{\max }=F_{\text {Nyquist }}$, (uSPAn, column 3), the usual stationary phase approximation truncated at $f_{\max }=F_{\mathrm{LSO}}$ (uSPAw, column 4), the corrected SPA (cSPA, column 5) and the improved SPA (inSPA, column 6), both extended up to $f_{\mathrm{Nyquist}}$, for different values of the total mass (column 1). The corresponding $F_{\mathrm{LSO}}$ is listed in column 2. [We deal only with equal mass binaries $\eta=1 / 4$.] In the last column we also list the total overlap 'intot' to exhibit the relative importance of the 'non-resonant' contribution. In this table and all others the smooth time-window starts at $30 \mathrm{~Hz}$; the seismic cutoff for LIGO is at $f_{\mathrm{s}}=40$ $\mathrm{Hz}$.

\begin{tabular}{|c|c|c|c|c|c|c|}
\hline$m / M_{\odot}$ & $F_{\mathrm{LSO}} / \mathrm{Hz}$ & $\overline{\left\langle\left\langle\tilde{h}_{\mathrm{N}}^{\mathrm{FFT}}, \tilde{h}_{\mathrm{N}}^{\mathrm{uSPAn}}\right\rangle\right.}$ & $\left\langle\tilde{h}_{\mathrm{N}}^{\mathrm{FFT}}, \tilde{h}_{\mathrm{N}}^{\mathrm{uSPAw}}\right\rangle$ & $\left\langle\tilde{h}_{\mathrm{N}}^{\mathrm{FFT}}, \tilde{h}_{\mathrm{N}}^{\mathrm{cSPA}}\right\rangle$ & $\left\langle\tilde{h}_{\mathrm{N}}^{\mathrm{FFT}}, \tilde{h}_{\mathrm{N}}^{\text {inSPA }}\right\rangle$ & $\overline{\left\langle\tilde{h}_{\mathrm{N}}^{\mathrm{FFT}}, \tilde{h}_{\mathrm{N}}^{\text {intot }}\right\rangle}$ \\
\hline 70.0 & 63 & 0.1536 & 0.6361 & 0.9231 & 0.9763 & 0.9944 \\
\hline 60.0 & 73 & 0.2294 & 0.7302 & 0.9489 & 0.9721 & 0.9873 \\
\hline 50.0 & 88 & 0.3336 & 0.8062 & 0.9724 & 0.9824 & 0.9934 \\
\hline 40.0 & 110 & 0.4708 & 0.8589 & 0.9862 & 0.9952 & 0.9993 \\
\hline 30.0 & 147 & 0.6682 & 0.9214 & 0.9964 & 0.9987 & 0.9977 \\
\hline 20.0 & 220 & 0.8811 & 0.9681 & 0.9968 & 0.9974 & 0.9986 \\
\hline 15.0 & 293 & 0.9431 & 0.9838 & 0.9999 & 0.9998 & 0.9997 \\
\hline 14.0 & 314 & 0.9528 & 0.9868 & 0.9995 & 0.9997 & 0.9993 \\
\hline 13.0 & 338 & 0.9641 & 0.9900 & 0.9986 & 0.9989 & 0.9993 \\
\hline 12.0 & 366 & 0.9700 & 0.9912 & 0.9996 & 0.9997 & 0.9995 \\
\hline 10.0 & 440 & 0.9839 & 0.9953 & 0.9989 & 0.9990 & 0.9994 \\
\hline 5.0 & 880 & 0.9983 & 0.9988 & 0.9991 & 0.9991 & 0.9992 \\
\hline 3.0 & 1466 & 0.9987 & 0.9987 & 0.9985 & 0.9985 & 0.9984 \\
\hline
\end{tabular}


TABLE III. Accuracy of the various approximations to the Fourier transform of a chirp signal computed in the test mass limit for P-approximants at different post-Newtonian orders $v^{n}$ (column 1) is measured by their overlaps with a waveform of exactly the same parameters but computed in the time-domain and then Fourier transformed using an FFT algorithm. The approximations considered are the usual SPAw (column 2), inSPAw (column 3) and irSPA (column 4) (with the choice $\left.x_{\text {up }}=0.36\right)$. Both uSPA and inSPA are windowed in frequency beyond $F_{\text {LSO }}$. Only the irSPA has a spectrum extending beyond $F_{\text {LSO }}$ though we put a numerical cutoff at $x_{\text {cutoff }}=-20 . x_{\text {up }}=0.36$ and $x_{\text {cutoff }}=-20$ in all subsequent tables.

\begin{tabular}{|c|c|c|c|}
\hline$n$ & uSPAw & inSPAw & irSPA \\
\hline & $\left\langle\tilde{h}_{\mathrm{P}_{n}}^{\mathrm{FFT}}, \tilde{h}_{\mathrm{P}_{n}}^{\mathrm{uSPAw}}\right\rangle$ & $\left.\begin{array}{l}\mathrm{FFT} \\
{ }_{n}\end{array} \tilde{h}_{\mathrm{P}_{n}}^{\text {inSPA }}\right\rangle$ & $\left\langle\tilde{h}_{\mathrm{P}_{n}}^{\mathrm{FFT}}, \tilde{h}_{\mathrm{P}_{n}}^{\mathrm{irSPA}}\right\rangle$ \\
\hline & \multicolumn{3}{|c|}{$m_{1}=1.4 M_{\odot}, m_{2}=10 M_{\odot}$} \\
\hline 4 & 0.9967 & 0.9986 & 0.9994 \\
\hline 5 & 0.9965 & 0.9990 & 0.9997 \\
\hline \multirow[t]{2}{*}{6} & 0.9965 & 0.9986 & 0.9993 \\
\hline & \multicolumn{3}{|c|}{$m_{1}=m_{2}=10 M_{\odot}$} \\
\hline 4 & 0.9764 & 0.9762 & 0.9951 \\
\hline 5 & 0.9771 & 0.9775 & 0.9955 \\
\hline \multirow[t]{2}{*}{6} & 0.9751 & 0.9786 & 0.9953 \\
\hline & \multicolumn{3}{|c|}{$m_{1}=m_{2}=20 M_{\odot}$} \\
\hline$\overline{4}$ & 0.8613 & 0.8613 & 0.9891 \\
\hline 5 & 0.8680 & 0.8773 & 0.9819 \\
\hline 6 & 0.8897 & 0.9038 & 0.9829 \\
\hline
\end{tabular}

TABLE IV. Faithfulness of the Fourier domain $P$-approximants in the formal test mass case. Values quoted are the minimax overlap (see DIS) of an approximant waveform generated directly in the frequency-domain with the exact waveform generated in the time-domain and Fourier transformed using the FFT algorithm. The approximations considered are the usual uSPAw (column 2) and irSPA (column 3) As mentioned earlier $x_{\text {up }}=0.36, x_{\text {cutoff }}=-20$. For compactness of presentation, in this Table and Tables $\mathrm{V}$ and $\mathrm{VI}$ we use for the column headings the abbreviation uSPAw to denote $\left\langle\tilde{h}_{\mathrm{X}}^{\mathrm{FFT}}, \tilde{h}_{\mathrm{P}}^{\mathrm{uSPAw}}\right\rangle$ irSPA to denote $\left\langle\tilde{h}_{\mathrm{X}}^{\mathrm{FFT}}, \tilde{h}_{\mathrm{P}_{n}}^{\mathrm{irSPA}}\right\rangle$.

\begin{tabular}{lcr|cc|ccc}
\hline \hline $\mathrm{n}$ & \multicolumn{2}{|c|}{$(1.4,10)$} & \multicolumn{2}{c|}{$(10,10)$} & \multicolumn{2}{c}{$(13,13)$} & $(20,20)$ \\
\hline & uSPAw & irSPA & uSPAw & irSPA & uSPAw & irSPA & uSPAw \\
\hline 4 & 0.8360 & 0.8316 & 0.9596 & 0.9707 & 0.9513 & 0.9965 & 0.8248 \\
5 & 0.9755 & 0.9729 & 0.9727 & 0.9914 & 0.9473 & 0.9973 & 0.8283 \\
6 & 0.9921 & 0.9903 & 0.9739 & 0.9938 & 0.9479 & 0.9972 & 0.8285 \\
\hline \hline
\end{tabular}

TABLE V. Faithfulness, as in Table IV but for waveforms constructed from post-Newtonian formulas for a binary with stars of comparable masses. The value of the parameter $\kappa_{0}=47 / 39$.

\begin{tabular}{lll|cc|cr|r}
\hline \hline $\mathrm{n}$ & \multicolumn{2}{|c|}{$(1.4,10)$} & \multicolumn{2}{c|}{$(10,10)$} & \multicolumn{2}{c|}{$(13,13)$} & $(20,20)$ \\
\hline & uSPAw & irSPA & uSPAw & irSPA & uSPAw & irSPA & uSPAw \\
\hline 4 & 0.7919 & 0.7898 & 0.9596 & 0.9584 & 0.9485 & 0.9665 & 0.8764 \\
5 & 0.9765 & 0.9736 & 0.9820 & 0.9924 & 0.9657 & 0.9921 & 0.8831 \\
6 & 0.9965 & 0.9958 & 0.9835 & 0.9947 & 0.9669 & 0.9921 & 0.8860 \\
\hline \hline
\end{tabular}


TABLE VI. Effectualness of the Fourier domain P-approximants in the formal test mass case. We list minimax overlaps of the approximate waveforms generated directly in the frequency-domain with the exact waveform generated in the time-domain and Fourier transformed using an FFT algorithm. However, in addition to maximisation over the lag parameter $\tau$ and the initial phase of the approximate waveform we also maximize over the two masses $m_{1}$ and $m_{2}$. The percentage bias in the estimation of the total mass $100\left(1-m_{A} / m\right)$ are listed in parenthesis below the overlaps.

\begin{tabular}{ccc|cc|ccc|c}
\hline \hline $\mathrm{n}$ & \multicolumn{2}{|c|}{$(1.4,10)$} & \multicolumn{2}{|c|}{$(10,10)$} & \multicolumn{2}{c|}{$(13,13)$} & $(20,20)$ \\
\hline & uSPAw & irSPA & uSPAw & irSPA & uSPAw & irSPA & uSPAw \\
\hline 4 & 0.9942 & 0.9963 & 0.9798 & 0.9984 & 0.9651 & 0.9979 & 0.9166 \\
& $(-2.300)$ & $(-2.744)$ & $(-0.664)$ & $(-0.858)$ & $(-0.962)$ & $(-1.081)$ & $(-2.500)$ & $(-1.250)$ \\
5 & 0.9882 & 0.9987 & 0.9795 & 0.9979 & 0.9653 & 0.9968 & 0.9174 & 0.9965 \\
& $(+0.480)$ & $(-1.301)$ & $(-0.626)$ & $(-0.467)$ & $(-0.631)$ & $(-0.481)$ & $(-1.875)$ & $(-0.340)$ \\
6 & 0.9970 & 0.9982 & 0.9805 & 0.9964 & 0.9649 & 0.9963 & 0.9174 & 0.9966 \\
& $(-0.311)$ & $(-1.166)$ & $(0.000)$ & $(-0.626)$ & $(-0.662)$ & $(0.000)$ & $(-1.250)$ & $(-0.340)$ \\
\hline \hline
\end{tabular}

TABLE VII. In this Table we list times required to generate templates first in the time-domain and then Fourier transforming using an FFT (column 2) and compare them with times required to construct the same templates directly in the Fourier domain using one of the three approximation schemes: uSPA (column 3), inSPA (column 4) and irSPA (5). For each post-Newtonian family (column 1) the time $t_{\mathrm{P}_{n}}^{\mathrm{FFT}}$ required to compute time-domain $\mathrm{P}$-approximant templates is the highest and we normalise all times by this value.

\begin{tabular}{|c|c|c|c|c|}
\hline$n$ & FFT & uSPA & inSPA & irSPA \\
\hline & $t_{\mathrm{P}_{n}}^{\mathrm{FFT}} / t_{\mathrm{P}_{n}}^{\mathrm{FFT}}$ & $t_{\mathrm{P}_{n}}^{\mathrm{uSPA}} / t_{\mathrm{P}_{n}}^{\mathrm{FFT}}$ & $t_{\mathrm{P}_{n}}^{\text {inSPA }} / t_{\mathrm{P}_{n}}^{\mathrm{FFT}}$ & $t_{\mathrm{P}_{n}}^{\mathrm{irSPA}} / t_{\mathrm{P}_{n}}^{\mathrm{FFT}}$ \\
\hline & \multicolumn{4}{|c|}{$m_{1}=1.4 M_{\odot}, m_{2}=10 M_{\odot}$} \\
\hline$\overline{4}$ & 1 & 0.013 & 0.021 & 0.089 \\
\hline \multirow[t]{2}{*}{6} & 1 & 0.014 & 0.021 & 0.090 \\
\hline & \multicolumn{4}{|c|}{$m_{1}=m_{2}=10 M_{\odot}$} \\
\hline 4 & 1 & 0.009 & 0.015 & 0.11 \\
\hline 6 & 1 & 0.009 & 0.016 & 0.11 \\
\hline
\end{tabular}


FIG. 1. This Figure compares the signal-to-noise ratio (SNR) for an inspiral signal searched by means of two different filters, in two different interferometers [initial LIGO, Eq. (1.4a) or VIRGO, Eq. (1.5a)]. The exact signal $h$ is assumed to be a time-truncated Newtonian chirp (sufficiently well approximated by the inSPA). The solid lines represent the optimal SNR, obtained when the filter $k$ is identical with the exact signal $h$. The dotted lines represent the sub-optimal SNR, obtained when the filter $k$ is the frequency-windowed usual SPA (uSPAw). In the case of LIGO, for low mass binaries uSPAw extracts the full SNR, but at higher masses, which are the most crucial ones for initial interferometers, it loses SNR up to a factor of 1.5 leading to a loss in the number of detectable events up to 70\%. In the case of VIRGO, uSPAw performs quite well when the total mass $m<50 M_{\odot}$, and requires inSPA for heavier binaries. The loss in the number of events caused by uSPAw for heavier mass binaries in the range $m>50 M_{\odot}$ is unacceptable.

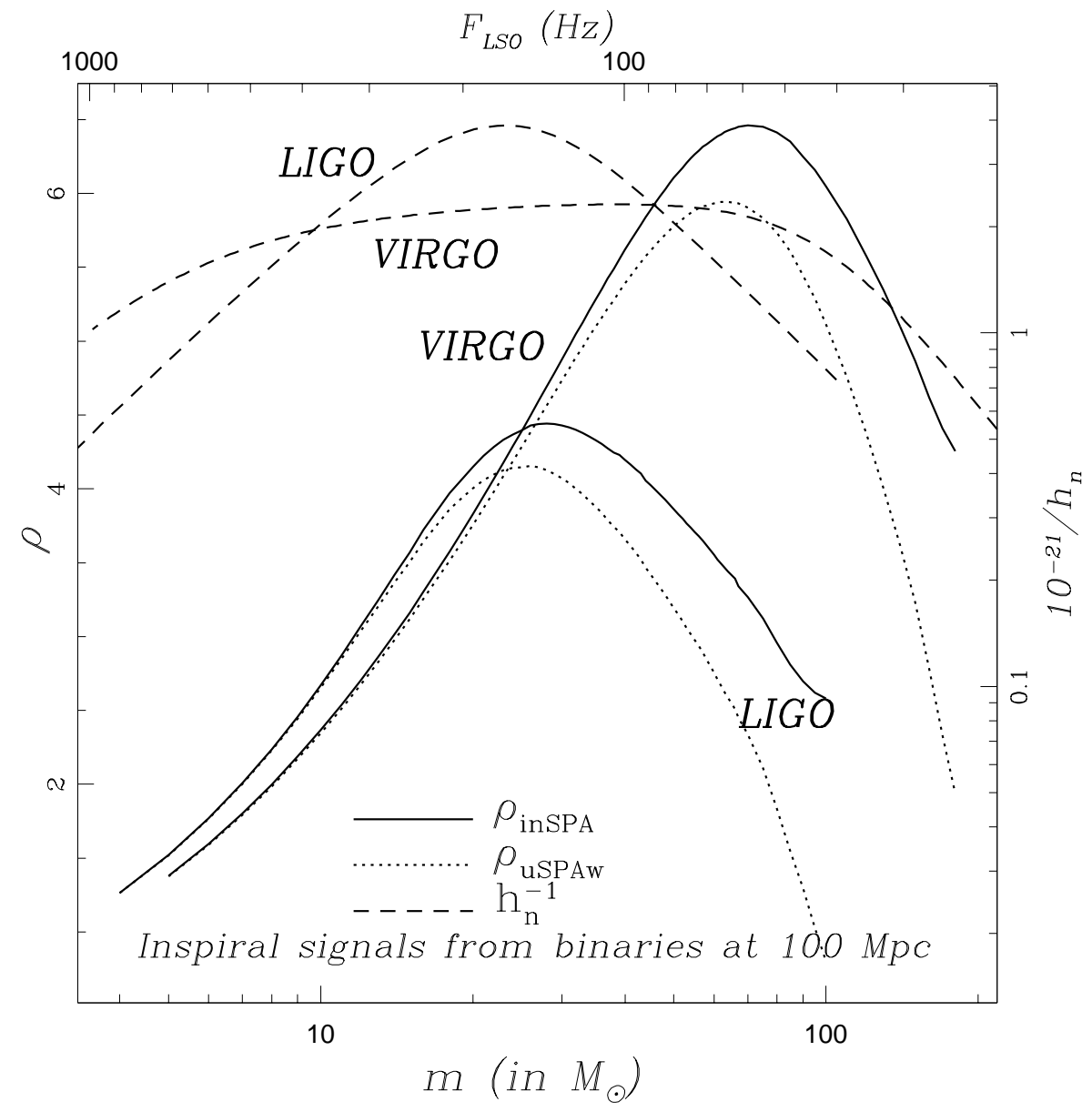


FIG. 2. The whitening kernel, $w_{\frac{1}{2}}(t)$, Eq. (2.10), is shown [for the initial LIGO noise curve, Eq. (1.4a)] plotted as a function of time. We see that it is quite sharply peaked at $t=0$. The peak drops quite rapidly as we move away from the origin thus indicating that we have almost local-in-time filters. The curve also indicates the importance of knowing the plunge signal over a time-scale of several 10's of milliseconds so that the inspiral signal can have a good overlap.

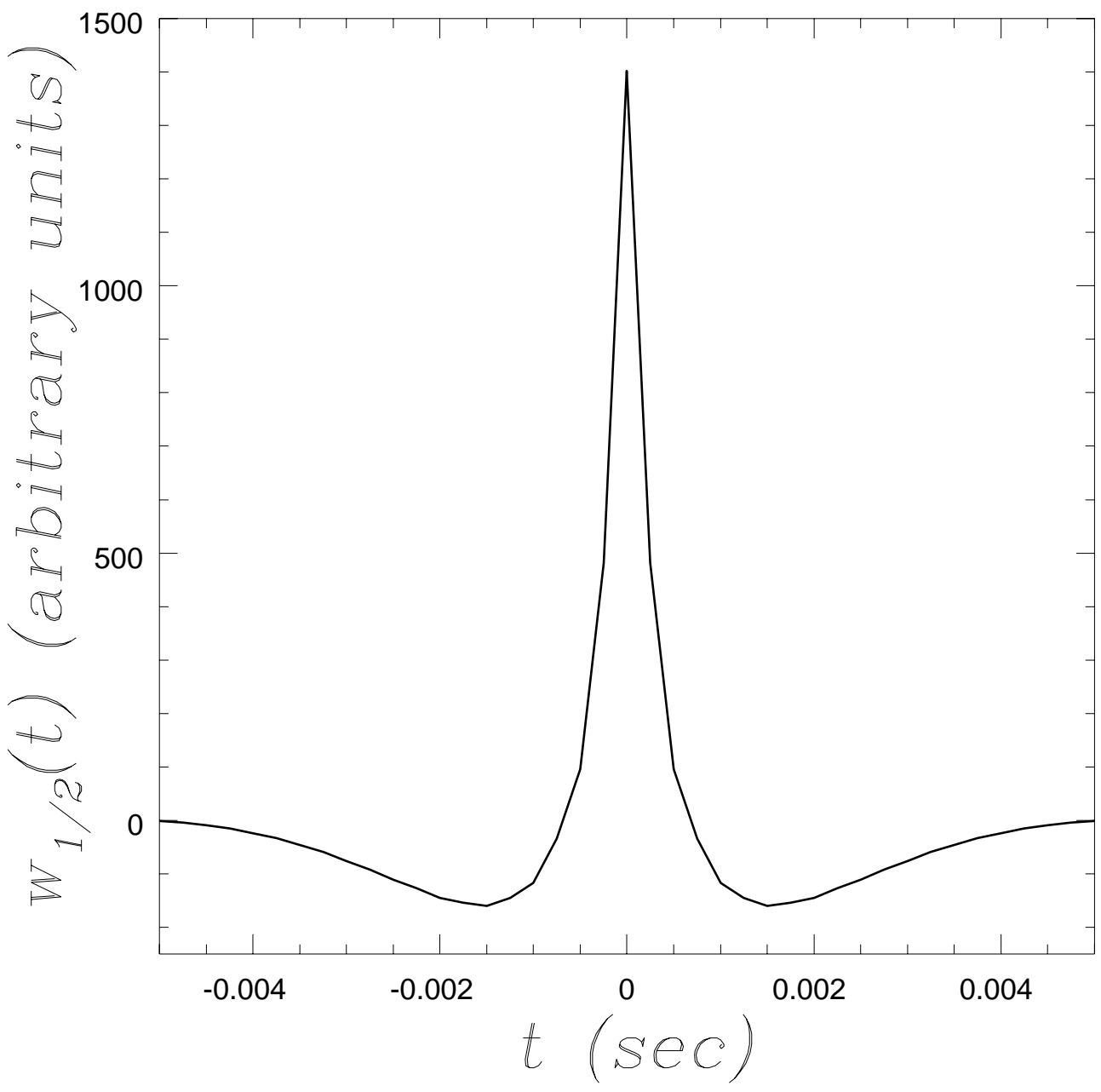


FIG. 3. The Newtonian instantaneous number of cycles $N(f)$, the square of the amplitude $a^{2}(f)$, their product $N(f) a^{2}(f)$, the reciprocal of the effective GW noise $h_{n}^{2}(f)=f S_{n}(f)$, and $d(S N R)^{2} / d(\log f)$ are plotted as a function of $f$. The scale on the y-axis corresponds to $N(f)$ on the left and all other functions are plotted on an arbitrary scale indicated on the right. The top panel is for a lighter mass binary $\left(m_{1}=1.4 M_{\odot}, m_{2}=10 M_{\odot}\right)$ and the lower one for a heavier mass system $\left(m_{1}=m_{2}=10 M_{\odot}\right)$.

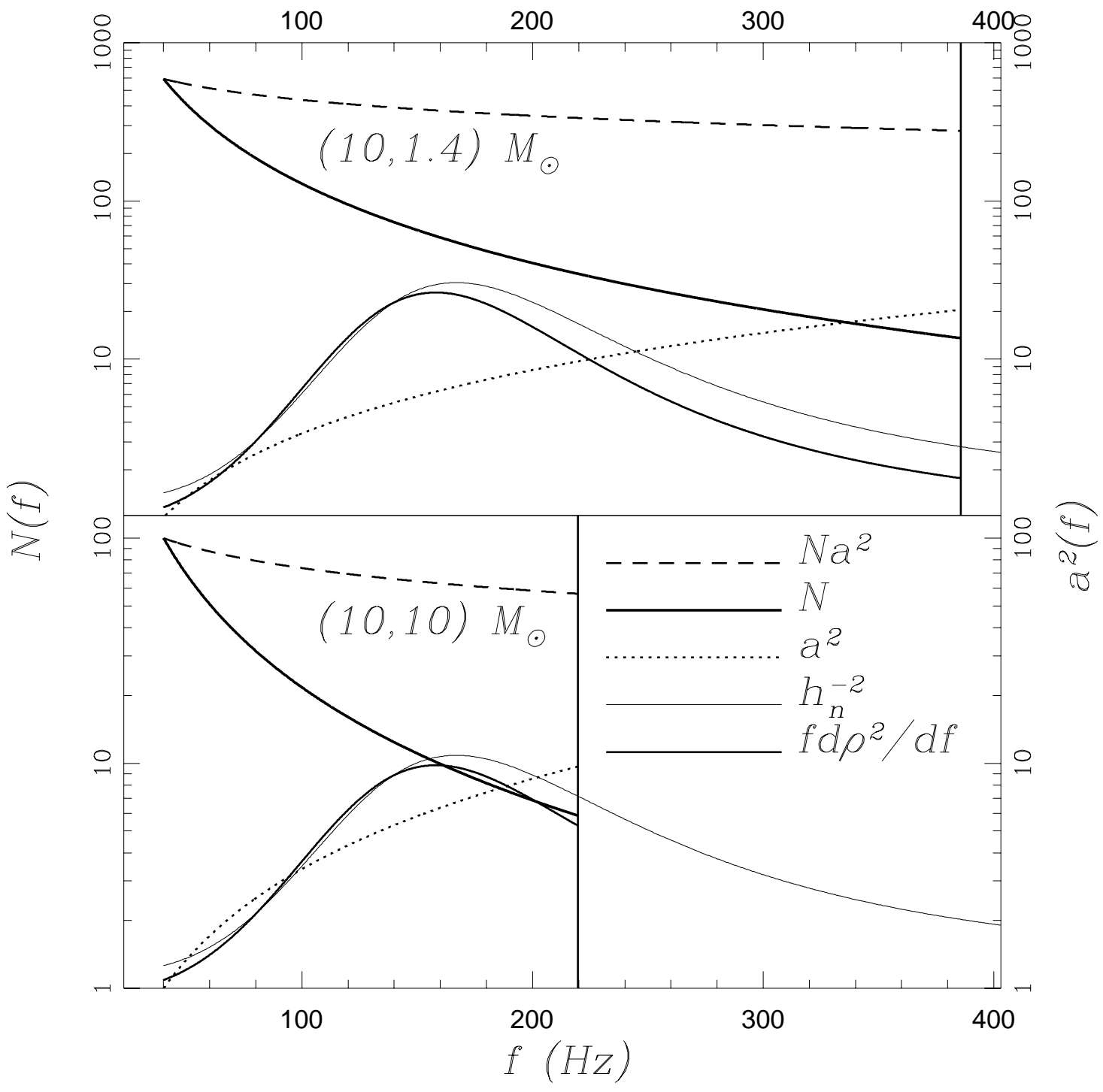


FIG. 4. Instantaneous number of cycles, Eq.(2.19), near the LSO as a function of time for the Newtonian case, Eq. (2.26), and the relativistic cases, Eq. (2.28), defined by the $2 \mathrm{PN}$ approximant $P_{4}$. Also plotted is the development of the wave-form $h_{P_{4}}(t)$ on an arbitrary scale. The plots show how the number of useful cycles diminishes as one gets close to the LSO. The Figure refers to $m_{1}=m_{2}=20 M_{\odot}$.

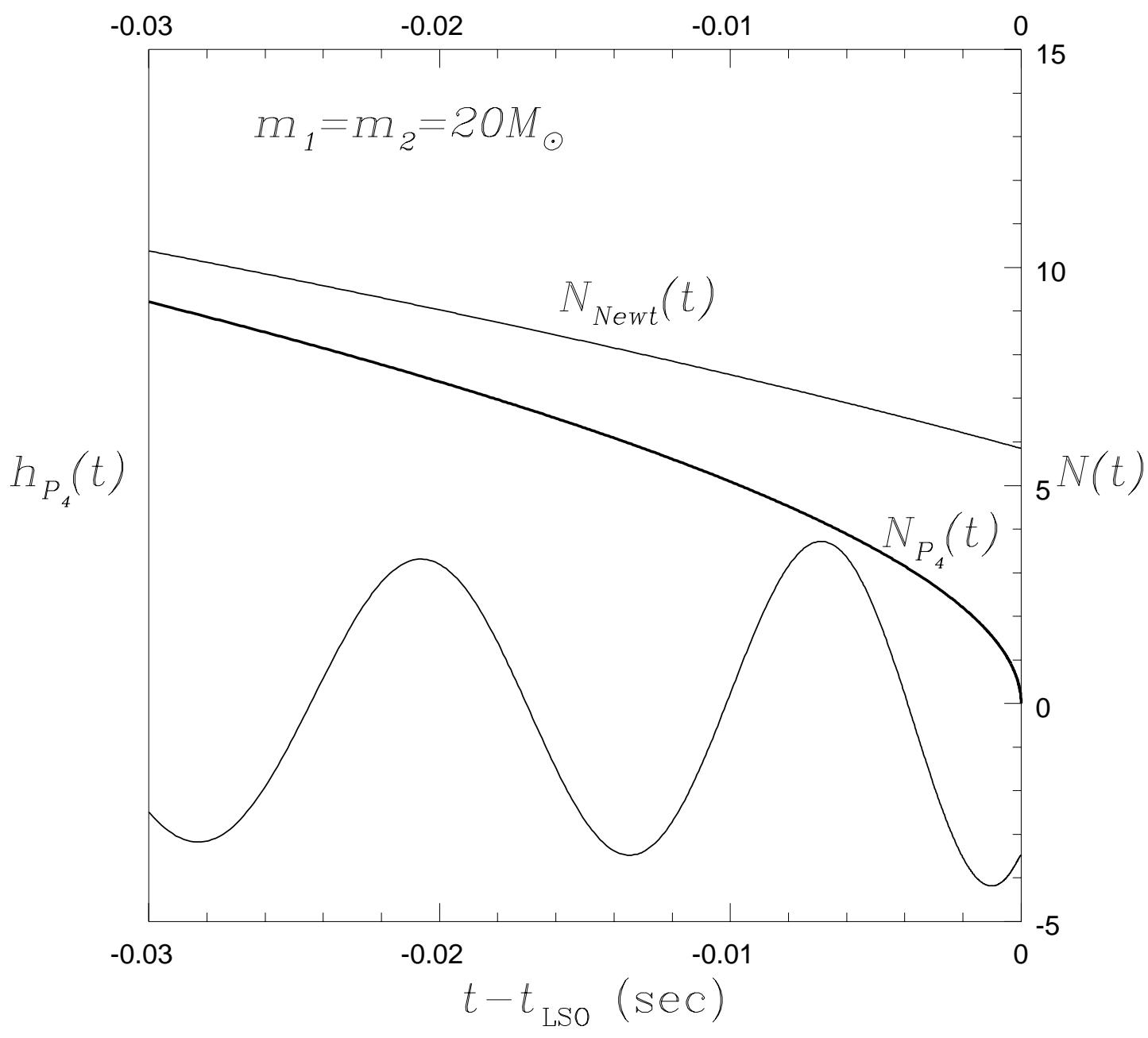


FIG. 5. The real and imaginary parts of the complex correction factor $\mathcal{C}(\zeta)$ - in terms of which the new frequency domain approximants are represented in the Newtonian-like cases — as a function of $\zeta$. The real part is a 'softened step' while the imaginary part an oscillatory function vanishing at the origin and at large positive and negative values of its argument.

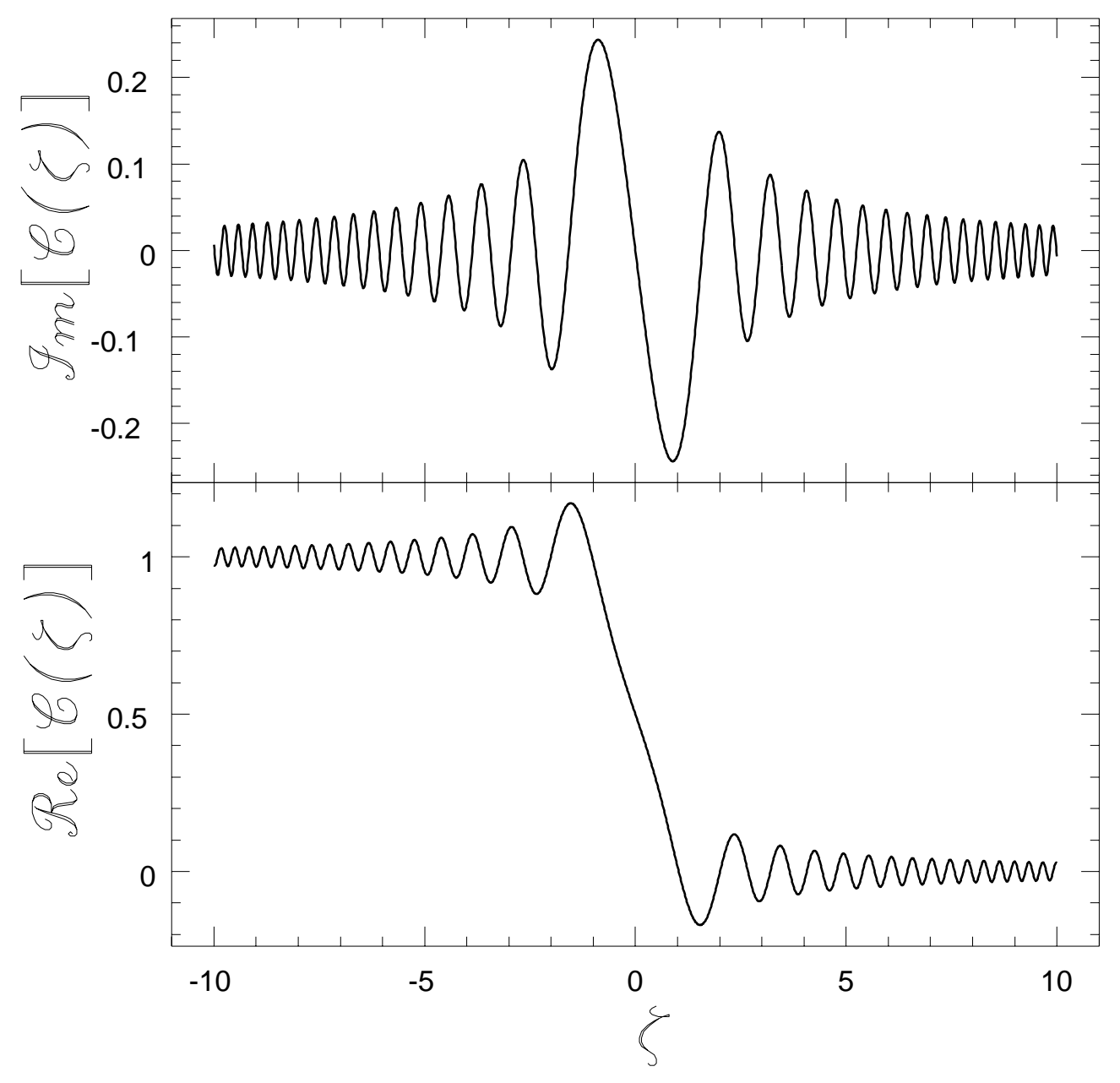


FIG. 6. The power per logarithmic bin of the squared SNR $d\left(\rho^{2}\right) / d(\log f)=f|\tilde{h}(f)|^{2} / S_{n}(f)$ for an arbitrarily normalised Newtonian signal computed from its DFT and its various approximate representations computed up to the Nyquist frequency: uSPAn, cSPAn and inSPAn. In the most sensitive range of frequencies, our final proposal inSPAn agrees with the FFT quite well. The uSPAn grossly overestimates the true signal power at frequencies $f>F_{\mathrm{LSO}}$. The last stable orbit frequency in this case $\left(m_{1}=m_{2}=10 M_{\odot}\right)$ is at about $220 \mathrm{~Hz}$ (the vertical line). Observe that, therefore, even uSPAw would significantly overestimate the signal power up to $F_{\mathrm{LSO}}$.

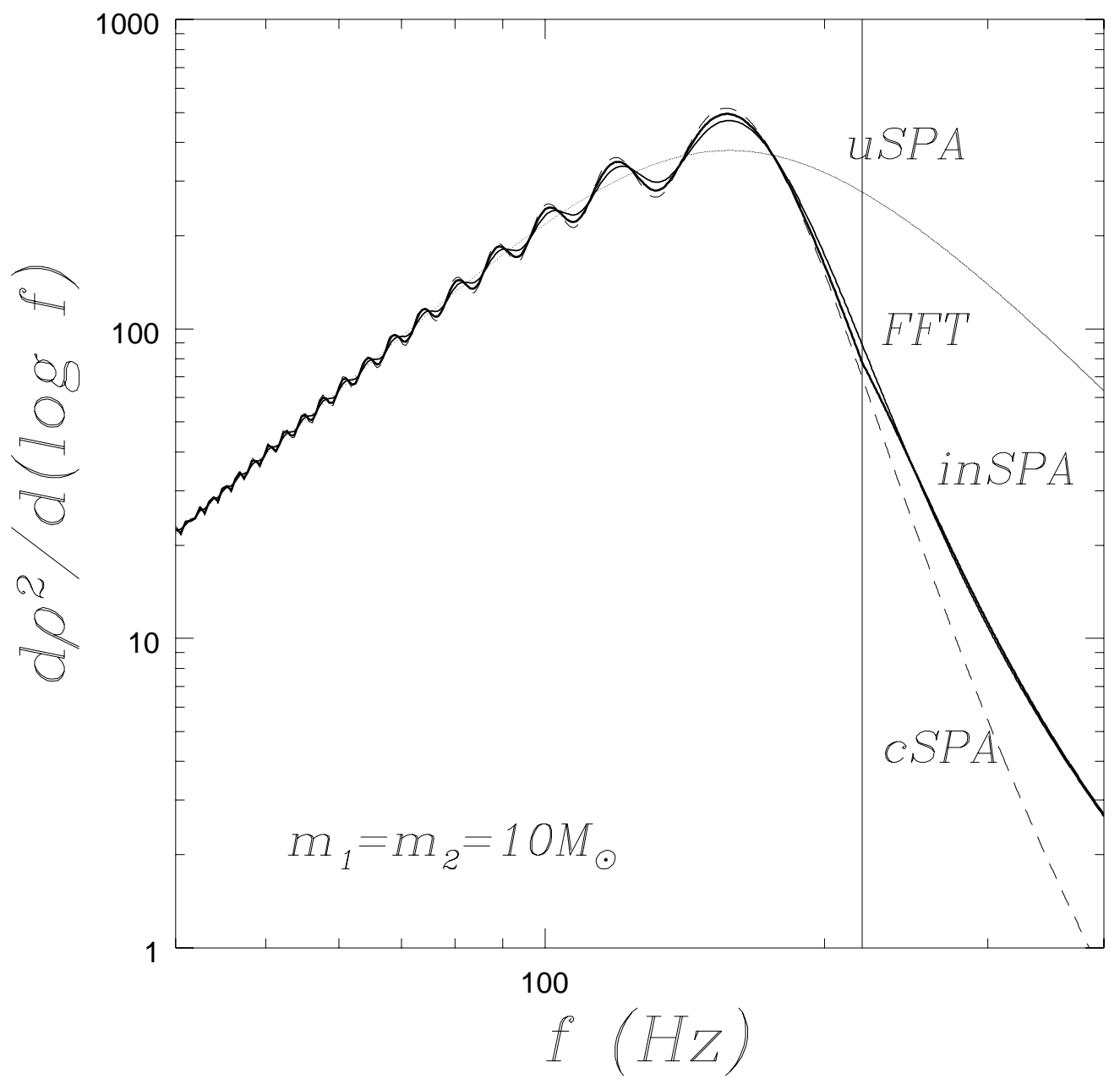


FIG. 7. The instantaneous GW frequency $F(t)$ versus $t$ for the Newtonian and relativistic cases during the last few orbits before the plunge at LSO. Notice the rapid increase in the inspiral rate close to the last stable orbit in the relativistic case.

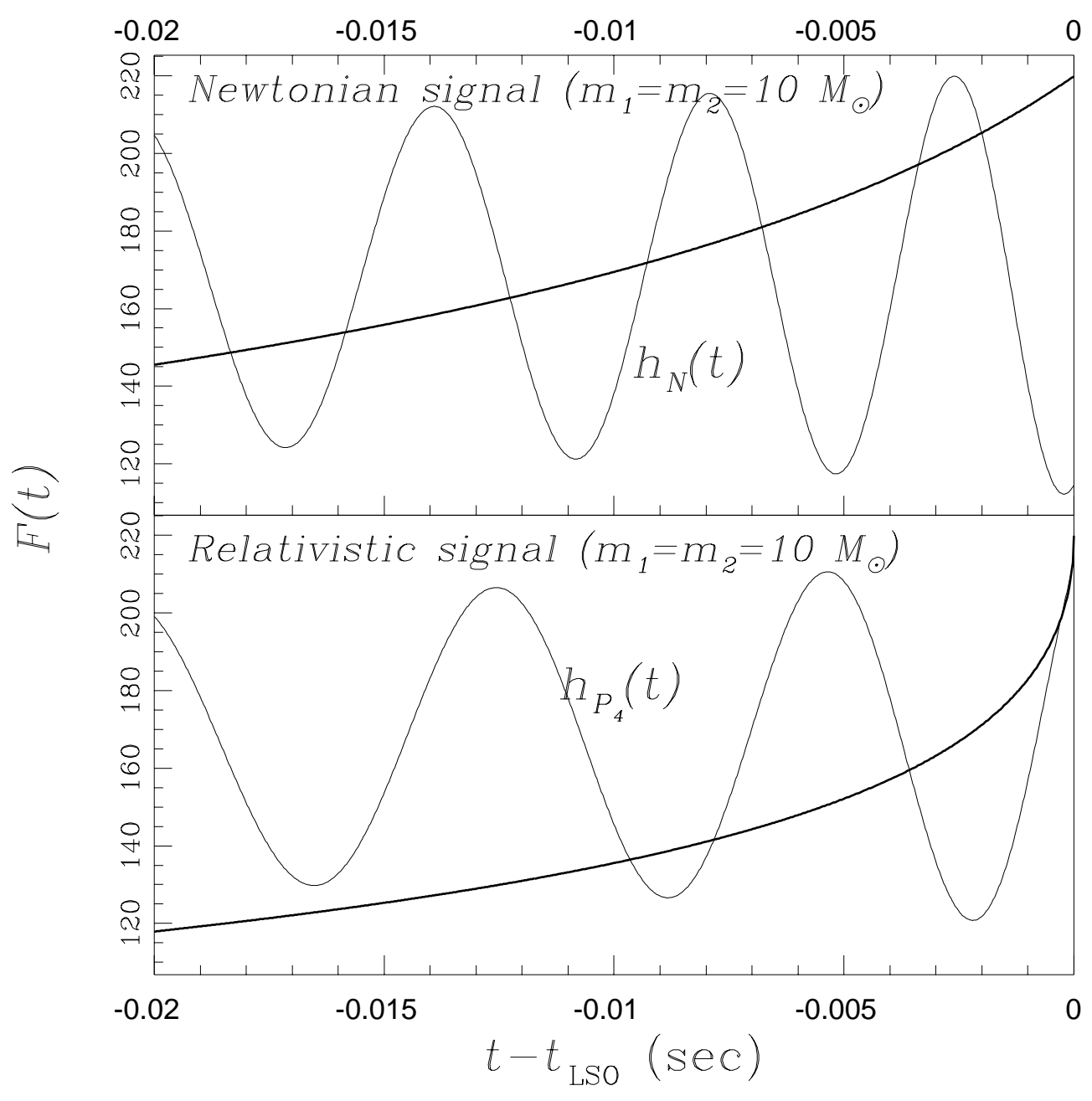


FIG. 8. The real and imaginary parts of the $g_{\frac{3}{2}}(x)$ function Eq. 4.37) in terms of which the improved relativistic SPA (irSPA) is represented. The thick lines represent the asymptotic behaviour as $x \rightarrow \pm \infty$ given by Eqs.(4.36b) and (4.36c).

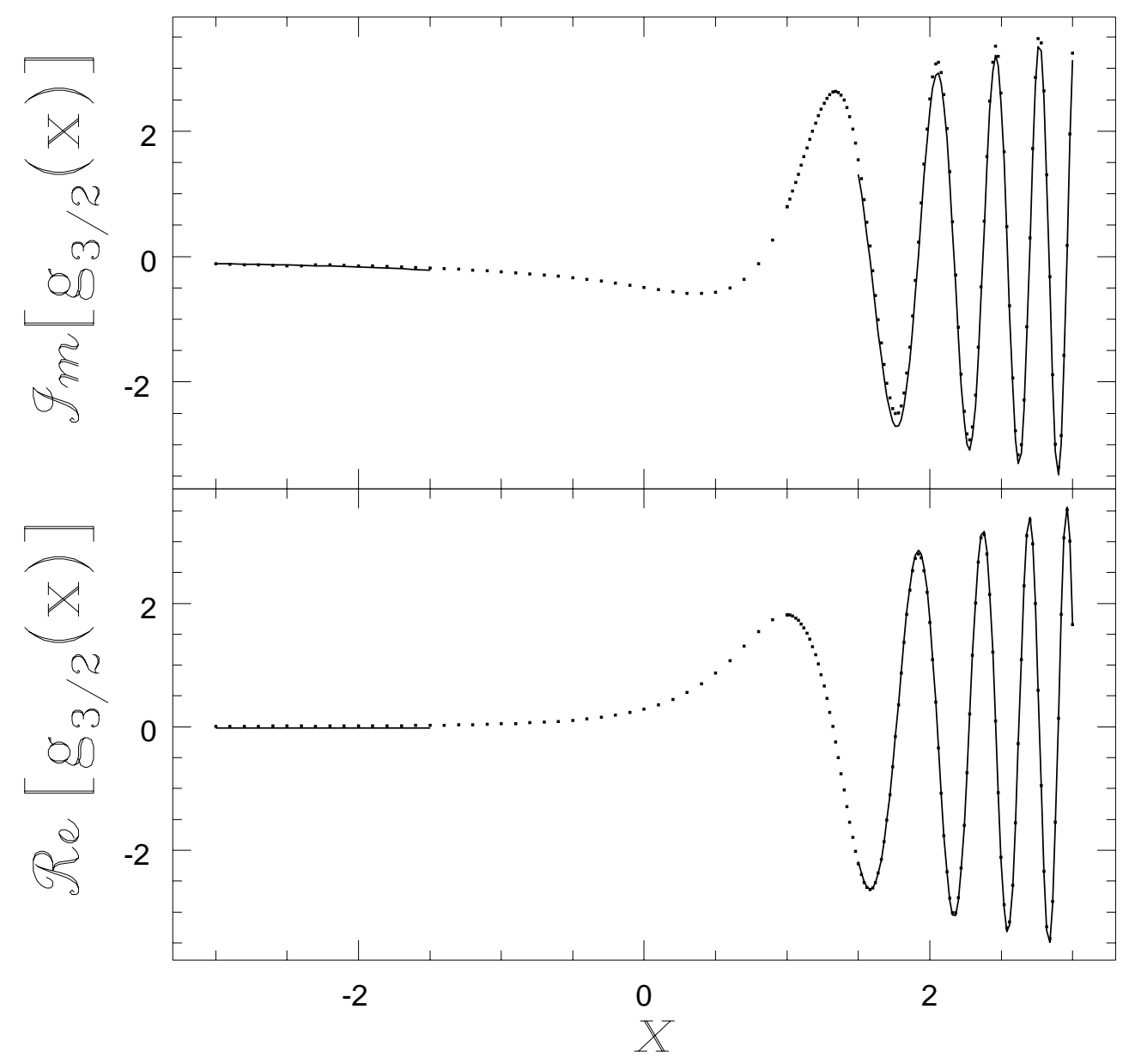


FIG. 9. Visual comparison of a chirp generated directly in the time-domain (solid line) with the inverse Fourier transforms of the usual SPA terminated at LSO (uSPAw) (dashed line) and the improved relativistic SPA (irSPA) extended up to the Nyquist frequency (dotted line), all during the last few cycles before the last stable circular orbit frequency is reached. The Figure corresponds to the relativistic (second post-Newtonian P-approximant case $\left(P_{4}\right)$ ) inspiral of a binary of total mass $m=40 M_{\odot}$.

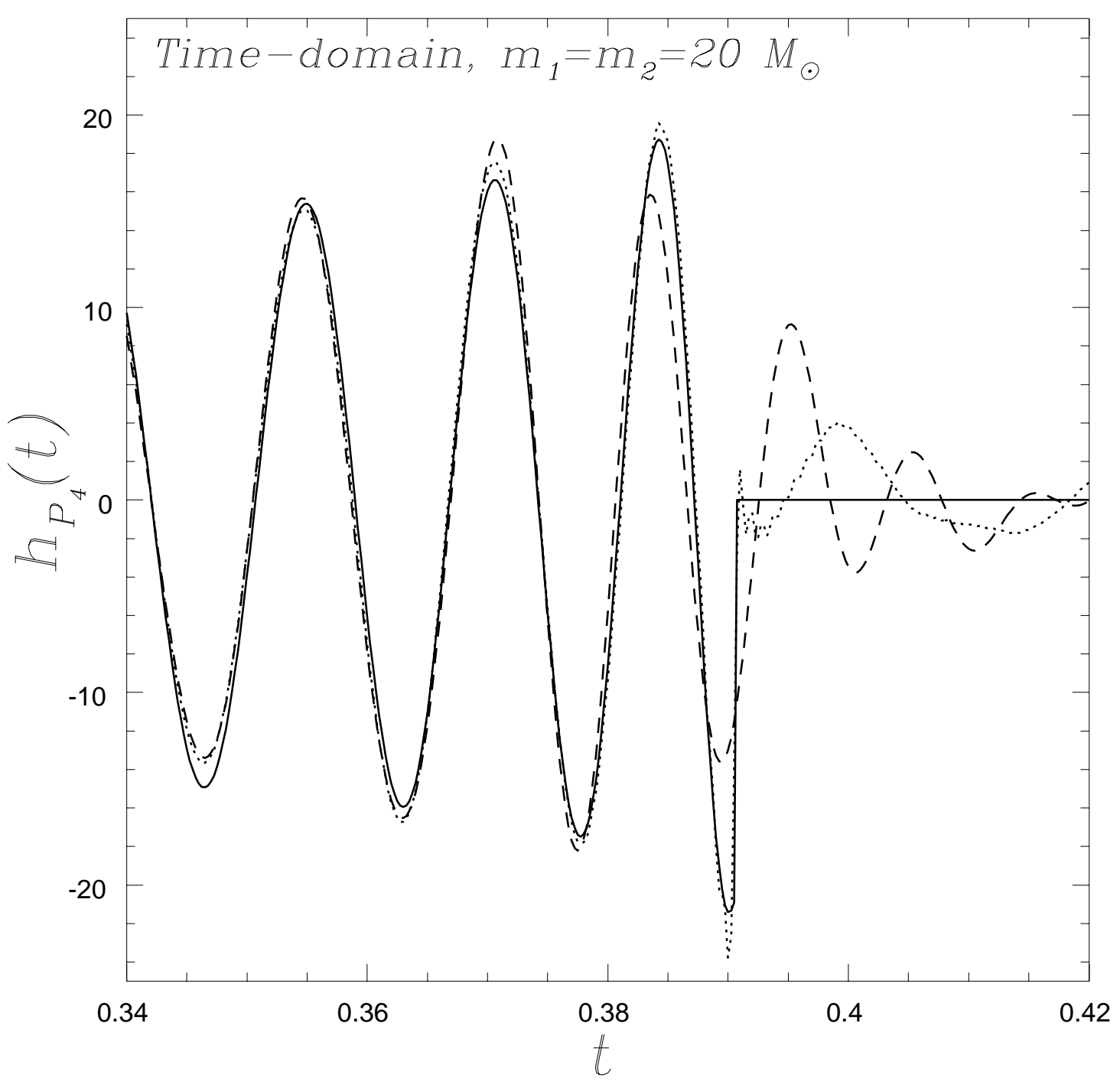


FIG. 10. This plot is the same as in Fig. 9 except that we compare whitened signals to show the effect of the detector response function on the time development. The inset shows the entire time-domain signal starting from $F_{\mathrm{GW}}=30 \mathrm{~Hz}$ and terminating at $F_{\mathrm{LSO}}$.

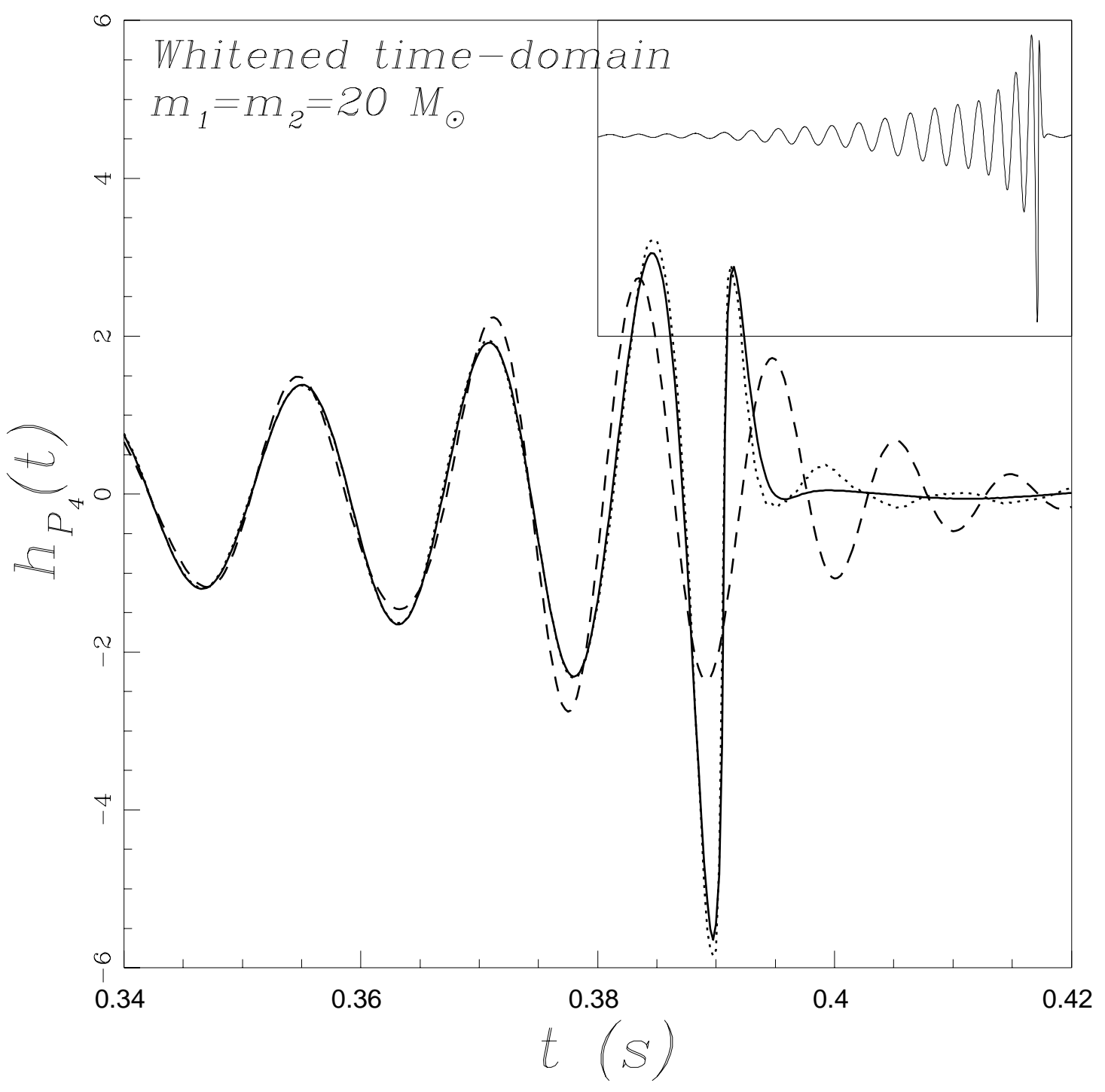

